\title{
Therapeutic Monoclonal Antibodies Targeting Immune Checkpoints for the Treatment of Solid Tumors
}

\author{
Nicholas Gravbrot ${ }^{1, *,+} \mathbb{D}$, Kacy Gilbert-Gard ${ }^{1, *,+}$, Paras Mehta ${ }^{1}\left(\mathbb{D}\right.$, Yarah Ghotmi ${ }^{1}$, \\ Madhulika Banerjee ${ }^{1}$, Christopher Mazis ${ }^{1}$ and Srinath Sundararajan ${ }^{1,2}$ \\ 1 Division of Hematology-Oncology, Department of Medicine, University of Arizona Cancer Center, Tucson, \\ AZ 85724, USA; parasmehta@email.arizona.edu (P.M.); yarahghotmi@email.arizona.edu (Y.G.); \\ mbanerje@email.arizona.edu (M.B.); mazisc@email.arizona.edu (C.M.); srinathshri@gmail.com (S.S.) \\ 2 Texas Oncology, Dallas, TX 75251, USA \\ * Correspondence: ngravbrot@email.arizona.edu (N.G.); kacyg@email.arizona.edu (K.G.-G.) \\ $+\quad$ N.G. and K.G.-G. contributed equally to this work.
}

Received: 24 September 2019; Accepted: 16 October 2019; Published: 21 October 2019

check for updates

\begin{abstract}
Recently, modulation of immune checkpoints has risen to prominence as a means to treat a number of solid malignancies, given the durable response seen in many patients and improved side effect profile compared to conventional chemotherapeutic agents. Several classes of immune checkpoint modulators have been developed. Here, we review current monoclonal antibodies directed against immune checkpoints that are employed in practice today. We discuss the history, mechanism, indications, and clinical data for each class of therapies. Furthermore, we review the challenges to durable tumor responses that are seen in some patients and discuss possible interventions to circumvent these barriers.
\end{abstract}

Keywords: immunotherapy; checkpoint inhibitor; monoclonal antibody; cancer therapy

\section{Introduction}

In recent years, the limitations of conventional chemotherapy have spurred research into more precise cancer treatment, using targeted therapies in hopes of selectively eradicating cancer while sparing normal host cells. As new cancer cell markers, cytokines, and immunologic checkpoints have been discovered, monoclonal antibodies $(\mathrm{mAb})$ and small molecule inhibitors have been developed to accomplish these goals. An important discovery in this area is that of immune checkpoint molecules, which dampen anti-cancer immune responses. Such proteins include programmed cell death protein-1 (PD-1), its ligands programmed death-ligand 1 and 2 (PD-L1, PD-L2) and cytotoxic T-lymphocyte-associated protein 4 (CTLA-4), among others. Inhibitors of immune checkpoints have since been developed as a means to "take the breaks off" of an otherwise impeded anti-cancer immune response. As additional targets have been discovered, new therapies have emerged. Herein, we review current $\mathrm{mAbs}$ directed at immune checkpoint modulation within the context of treating various solid cancers.

\section{Immunotherapy Overview}

\subsection{Overview}

Several classes of checkpoint modulators have been studied clinically. A summary of those with US Food and Drug Administration (FDA) approval is provided in Table A1. Table A1 additionally includes immunologic and pharmacologic parameters, such as IgG subtype, target affinity, and epitope 
properties, for each drug. Figure 1 provides an overview of implicated receptor-ligand interactions and their generalizable effects on the immune response.

For some of the mAbs discussed (such as CTLA-4 inhibitors), the exact mechanism of action is not fully understood and is the subject of active investigation. Therefore, the mechanisms presented represent the basic foundation of a presumably more complicated biochemical picture.

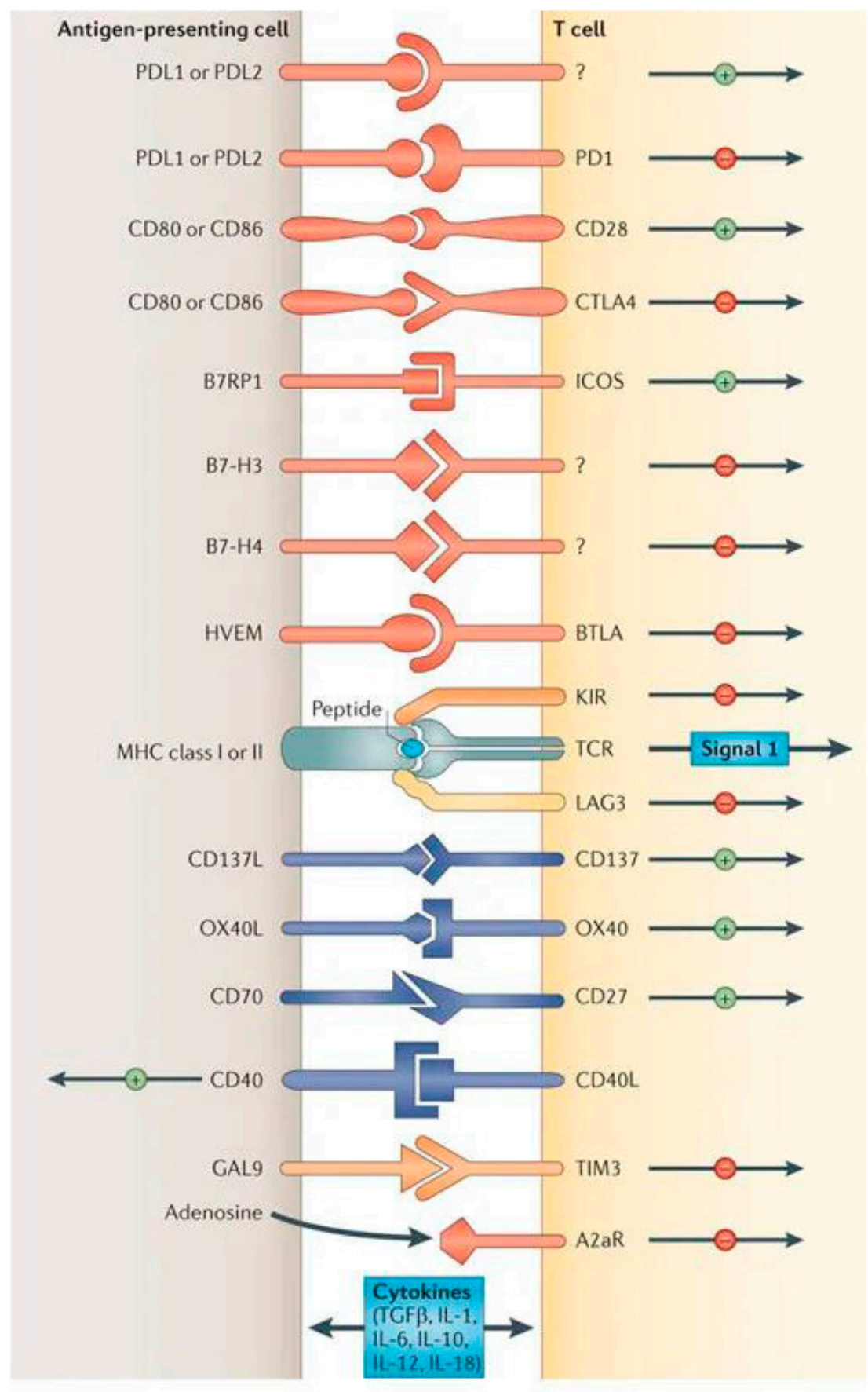

Nature Reviews | Cancer

Figure 1. Overview of receptor-ligand interactions involved in checkpoint modulation. Description: Examples of different receptors and ligands involved in checkpoint modulation, along with generalized stimulatory (+) or inhibitory (-) effects. Reprinted with permission from: Spinger Nature: Pardoll, D.M. The blockade of immune checkpoints in cancer immunotherapy. Nat. Cancer Rev. 2012, 12, 252-264. 


\subsection{CTLA-4 Inhibitors}

\subsubsection{Background}

Discovered in 1987, CTLA-4 was identified as a homolog of CD28, and later, an inhibitor to T cell activation [1,2]. After several years of preclinical and clinical trials, the first CTLA-4 inhibiting mAb, ipilimumab (Yervoy ${ }^{\circledR}$; BMS-734016; MDX-010; Bristol-Myers Squibb), gained FDA approval in 2011 for the treatment of unresectable or metastatic melanoma. A second CTLA-4 mAb, tremelimumab (CP-675,206; AstraZeneca), has also been developed.

\subsubsection{Mechanism of Action}

CTLA-4 is a homolog of CD28 with opposing functions. Both are expressed by T cells and bind the ligands B7-1 and B7-2 found on antigen-presenting cells (APC). When CD28 binds B7-1 and B7-2, intracellular signaling via phosphatidyl-inositol 3-kinase (PI3K) results in downstream activation of transcriptional factors that increase proliferation, differentiation, and survival of $\mathrm{T}$ cells [3]. The binding of B7-1 or B7-2 to CTLA-4, however, prohibits this response. CTLA-4 has a higher affinity for B7-1/B7-2 ligands relative to $C D 28$, and as such, it can outcompete CD28, allowing for suboptimal stimulation of T cells. CTLA-4 is constitutively expressed on regulatory T cells (Treg) and plays an important role in immune system suppression.

Ipilimumab and tremelimumab are monoclonal $\operatorname{IgG}$ antibodies targeted against CTLA-4. Ipilimumab is of the IgG1 subclass, whereas tremelimumab is an IgG2 molecule. Both have similar binding affinity to CTLA-4, though ipilimumab has a higher dissociation rate. Epitopes are also comparable between mAbs, as both bind to the F and G strands of the CTLA-4 molecule [4]. The inhibitory effect of either mAb against CTLA-4 facilitates increased CD28/B7 binding, thus upregulating $\mathrm{T}$ cell proliferation and immune activity $[5,6]$. A proposed secondary mechanism of CTLA-4 mAbs is that of Treg depletion within the tumor microenvironment (TME), as some studies have shown that decrease in Tregs with anti-CTLA-4 therapy [7-10]. However, several other studies have reported data that contradicts these findings, with no evidence of Treg depletion [11-14]. Therefore, this mechanism is still under investigation.

\subsubsection{Indications}

Currently, ipilimumab is the only anti-CTLA-4 mAb with FDA approval. It was first approved in 2011 for the treatment of unresectable or metastatic melanoma, and since then, its indications have expanded significantly (Table A1). Tremelimumab is undergoing investigation in various solid malignancies but has not yet been granted FDA approval so far. Figure 2 provides a timeline of noteworthy changes in FDA approvals for ipilimumab.

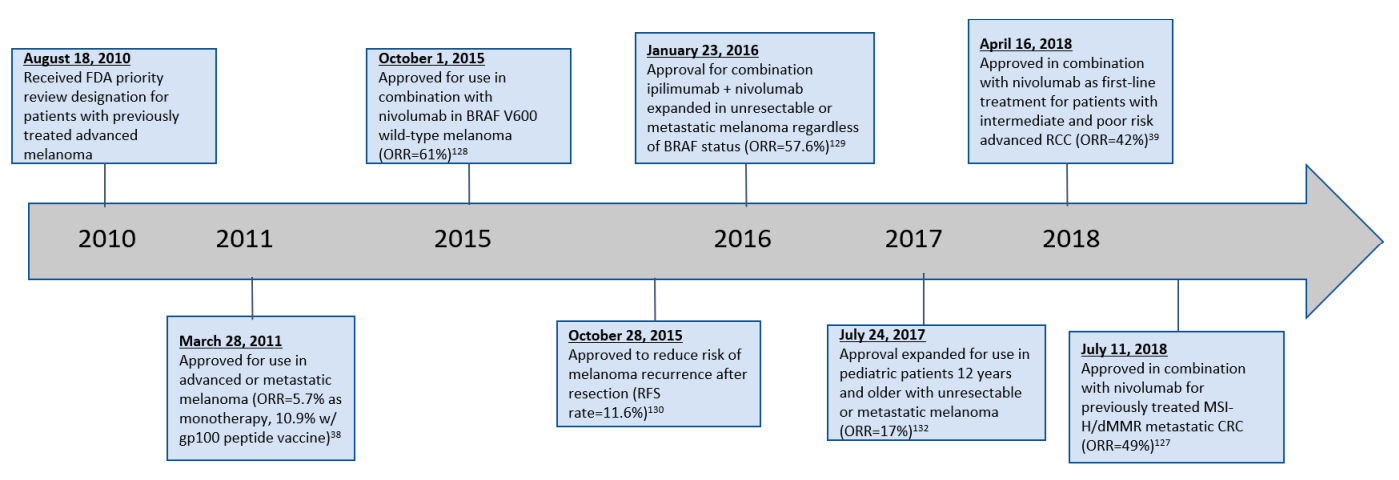

Figure 2. Timeline of ipilimumab FDA approvals. 


\subsection{PD-1 Inhibitors}

\subsubsection{Background}

The first phase I trial investigating anti-PD-1 mAbs was reported in 2012, and the field has grown immensely since then [15]. Nivolumab (Opdivo ${ }^{\circledR}$; BMS-936558; Bristol-Myers Squibb) and pembrolizumab (Keytruda ${ }^{\circledR}$; MK-3475; Merck) represent the most well-studied PD-1 inhibitors, though several other mAbs within this class exist.

\subsubsection{Mechanism of Action}

PD-1 is a type I membrane protein that is loosely related to CTLA-4 structurally [16]. It is expressed on activated T cells, B cells, and macrophages [17]. Under physiologic conditions, PD-1 negatively regulates $\mathrm{T}$ cell activity to maintain peripheral immune tolerance and to avoid immunopathology. It accomplishes this by binding to its ligands, PD-L1 and PD-L2, resulting in activation of an intracellular phosphatase, which, in turn, inhibits downstream kinase signaling customarily involved in $\mathrm{T}$ cell activation. Therefore, this results in decreased $\mathrm{T}$ cell proliferation and response $[18,19]$.

As with CTLA-4 inhibitors, PD-1 inhibitors work by enhancing the patient's natural anti-tumor immune response. IgG mAbs that block PD-1, such as nivolumab and pembrolizumab, inhibit the intracellular signaling cascade described above, resulting in disinhibition of the anti-tumor immune response. PD-1 inhibitors also block interactions with PD-L2. It should be noted that this is an advantage over PD-L1 inhibitors, which do not block PD-1/PD-L2 interactions [20].

Both nivolumab and pembrolizumab are of the IgG4 subclass, but the epitope binding regions of each differ. For nivolumab, its epitope is predominantly in the PD-1 N-loop, whereas pembrolizumab's epitope primarily involves the PD-1 CD loop. Both have high affinity and high specificity for PD-1 [21].

\subsubsection{Indications}

In late 2014, both nivolumab and pembrolizumab received their first FDA-approved indications for use in unresectable or metastatic melanoma in patients with history of disease progression following ipilimumab and, if BRAF V600 mutation positive, BRAF inhibition. Since then, the scope of anti-PD-1 therapy has broadened tremendously (Table A1). A timeline of changes in FDA-approved indications is provided in Figure 3 for nivolumab and Figure 4 for pembrolizumab.

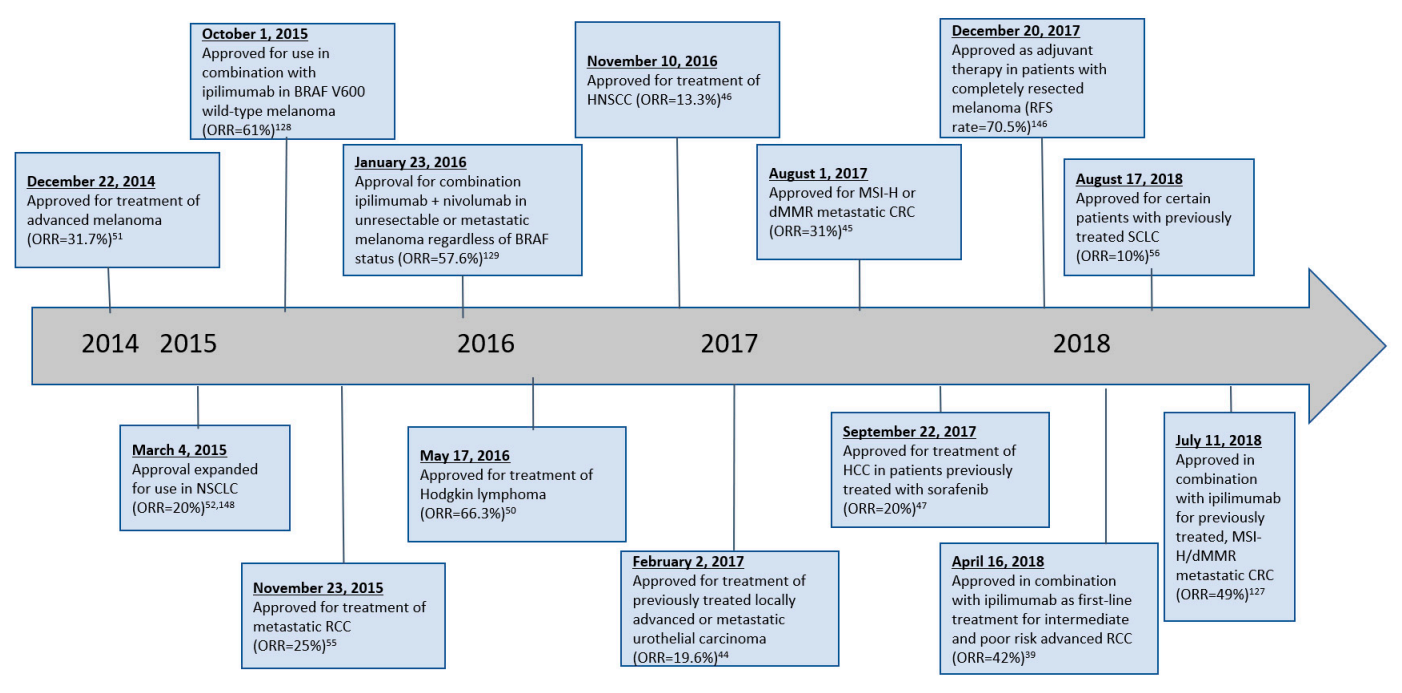

Figure 3. Timeline of nivolumab FDA approvals. 


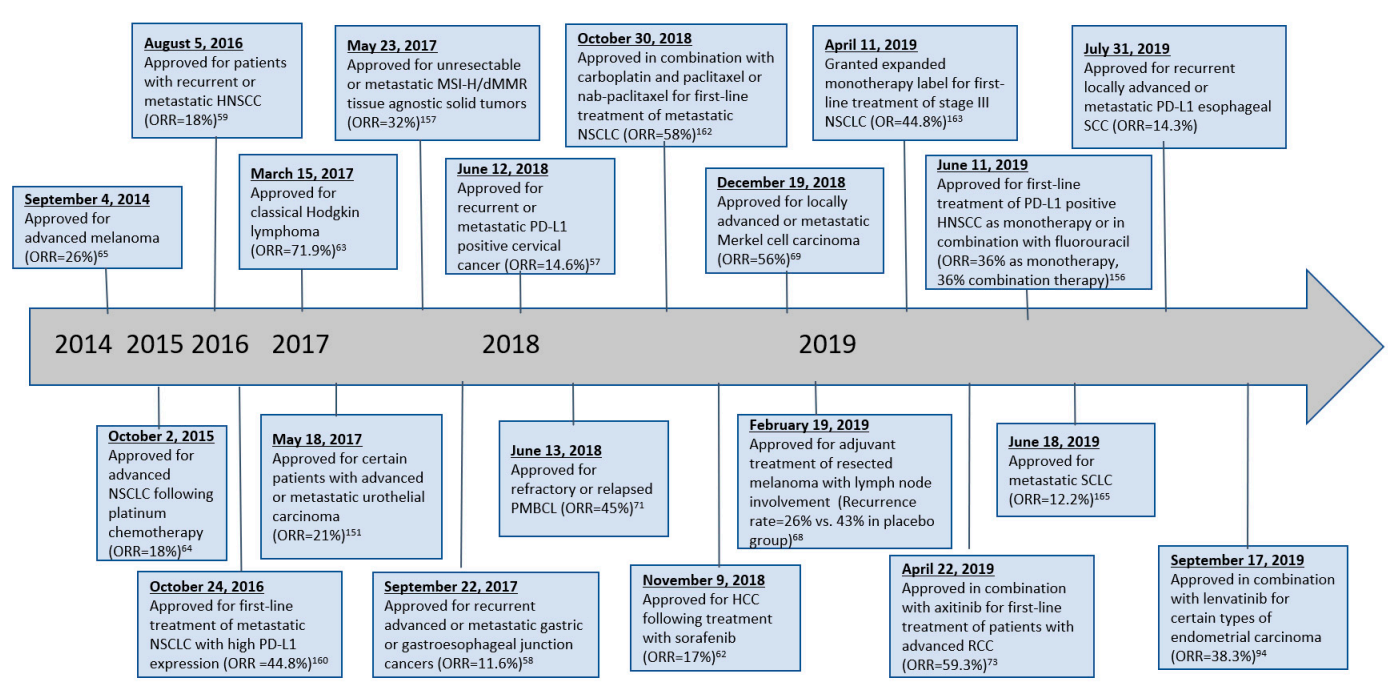

Figure 4. Timeline of pembrolizumab FDA approvals.

\subsection{PD-L1 Inhibitors}

\subsubsection{Background}

While anti-PD-1 therapies "blazed the trail" for modulation of the PD-1/PD-L1 axis, research into mAbs directed against PD-L1 followed shortly after. Atezolizumab (Tecentriq ${ }^{\circledR}$; Genetech/Roche) has garnered the most investigation within the class, but others include durvalumab (Imfinzi ${ }^{\circledR}$; Medimmune/AstraZeneca) and avelumab (Bavencio ${ }^{\circledR} ;$ Merck/Pfizer).

\subsubsection{Mechanism of Action}

PD-L1 is a type I transmembrane protein expressed by T cells, B cells, natural killer (NK) cells, macrophages, dendritic cells, and epithelial cells; pathologically, PD-L1 can directly be expressed by cancer cells [22]. The effects of PD-L1/PD-1 interactions have been discussed previously. Like PD-1 inhibitors, PD-L1 inhibitors prevent ligand-receptor binding, blocking the immune suppressive effects mediated through this pathway. In addition to PD-1/PD-L1 interactions, PD-L1 is also known to bind competitively to B7-1, with similar effects as CTLA-4/B7-1 binding. Thus, PD-L1 inhibitors also block interactions between PD-L1 and B7-1, which further disinhibits anti-cancer immunity [20].

Atezolizumab, avelumab, and durvalumab are all IgG1 mAbs [23]. Each has a different epitope but share key interactions within PD-L1's CC'FG $\beta$-sheet. Unique interactions involve regions within the BC, $\mathrm{CC}^{\prime}, \mathrm{C}^{\prime} \mathrm{C}^{\prime \prime}$ and FG loops (atezolizumab); $\mathrm{CC}^{\prime}$ loop and N-terminus (durvalumab); and $\mathrm{CC}^{\prime}$ loop (avelumab). All have been shown to have dissociation constants of less than $1 \mathrm{nM}$, indicating high affinity for PD-L1.

\subsubsection{Indications}

The first PD-L1 mAb to be granted FDA approval was atezolizumab, which received accelerated approval in May 2016 for the treatment of locally advanced or metastatic urothelial cancer resistant to platinum-containing chemotherapy regimens. Several other approvals have been granted over time (Table A1). Figure 5 provides a summary of changes in FDA-approved indications for the various PD-L1 mAbs. 


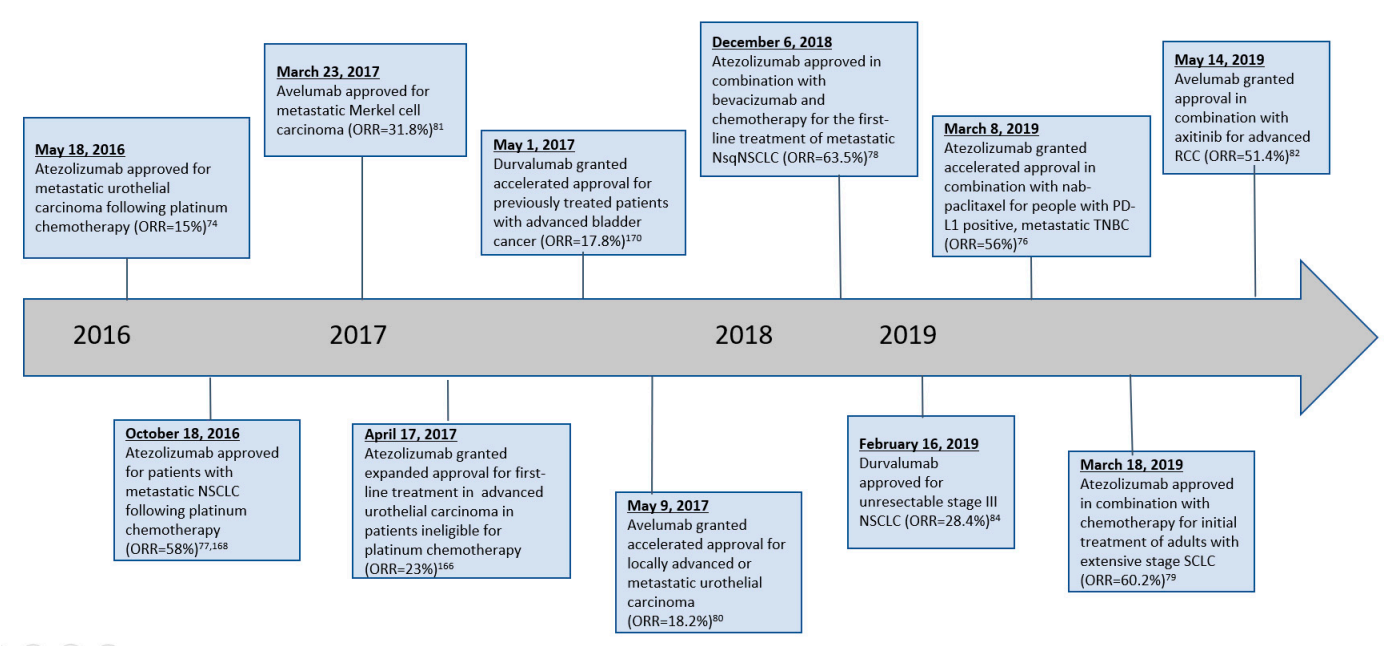

Figure 5. Timeline of PD-L1 inhibitor FDA approvals.

\subsection{LAG-3 Inhibitors}

\subsubsection{Background}

While lymphocyte-activation gene-3 (LAG-3) modulation dates back as far as 2006 with the LAG-3-immunoglobulin fusion protein eftilagimod alpha (IMP321; Immutep), the earliest mAb directed against LAG-3 was relatlimab (BMS-986016; Bristol-Myers Squibb), which was first evaluated in the treatment of several solid malignancies from 2013-2017 [24]. Relatlimab continues to be the subject of interest in several active clinical trials.

\subsubsection{Mechanism of Action}

LAG-3 is a type I transmembrane protein expressed by activated T cells, B cells, NK cells and dendritic cells, and it is involved in negative regulation of helper and cytotoxic T cell responses [25-27]. Activation of LAG-3 occurs peripherally via binding to class II major histocompatibility complex (MHC) and/or galectin-3 [27]. Activated LAG-3 reduces production of various immunostimulatory interleukins (IL) and enhances sensitivity to Treg signaling, thus increasing tolerance and accelerating $\mathrm{T}$ cell exhaustion [27,28]. Anti-LAG-3 antibodies prevent binding of LAG-3 with its ligands, blocking these effects and thereby facilitating increased anti-tumor activity.

\subsubsection{Indications}

There are no FDA-approved indications thus far for anti-LAG-3 mAbs. Investigations in various solid and hematologic malignancies are ongoing, with a phase II/III study representing the highest stage of development.

\subsection{TIM-3 Inhibitors}

\subsubsection{Background}

$\mathrm{T}$ cell immunoglobulin and mucin-domain containing-3 (TIM-3) mAbs are currently under investigation in early clinical studies, with the first phase I trial having opened in 2015. Agents in this class include Sym023 (Symphogen), TSR-022 (Tesaro/AnaptysBio), MBG453 (Novartis), LY3321367 (Lilly), and BGB-A425 (BeiGene).

\subsubsection{Mechanism of Action}

TIM-3 is a type I transmembrane protein implicated in suppression of $\mathrm{T}$ cell-mediated immune responses. TIM-3 downregulates the production of several cytokines, including IL-2, IL-12, 
interferon-beta, and interferon-gamma, and additionally expedites $\mathrm{T}$ cell exhaustion [29,30]. Given the possibility for expression in multiple cell lines, TIM-3 may dampen T cell activation at varying stages of the immune response, even upstream to direct $\mathrm{T}$ cell interactions [29]. TIM-3 inhibitors block these responses, mitigating the immune inhibition mediated through this pathway. As with the other checkpoint inhibitors, this disinhibition results in an enhanced anti-tumor immune response.

\subsubsection{Indications}

There are no current FDA-approved indications for anti-TIM-3 mAbs. A number of early studies assessing anti-TIM-3 therapy in localized or advanced solid malignancies are ongoing.

\section{7. $C D 40$ Agonists}

\subsubsection{Background}

Immune checkpoint blockade is often ineffective when severe immunosuppression develops; thus, targets that further promote an intratumoral $\mathrm{T}$ cell response are needed [31]. One such target is CD40, a member of the tumor necrosis factor (TNF) receptor superfamily expressed on both immune and non-immune cells [32]. Therapies within this class include CP-870,893 (RG-7876; Pfizer/Roche) and APX005M (Apexigen).

\subsubsection{Mechanism of Action}

CD40 is a cell surface molecule of the TNF receptor superfamily that is present physiologically on APCs and pathologically on tumor cells [33]. The CD40 ligand (CD40L/CD154) is expressed on activated CD4+ and CD8+ T cells, B cells and NK cells [34]. CD40/CD40L signaling serves as the bridge between innate and adaptive immunity; CD40 expression on APCs enhances antigen presentation and co-stimulatory capacity, resulting in a robust activation of cytotoxic $\mathrm{T}$ cells even in the absence of a $\mathrm{CD} 4+\mathrm{T}$ cell helper signal [34]. Thus, $\mathrm{mAbs}$ acting as agonists for $\mathrm{CD} 40$ result in vigorous immunologic activation and proliferation.

\subsubsection{Indications}

There are no current FDA-approved indications for CD40 agonists. A number of early studies are ongoing.

\subsection{OX40 Agonists}

\subsubsection{Background}

Another member of the TNF receptor superfamily is OX40 (CD134), which has similar functions. Therapeutic OX40 agonists include 9B12 (Providence Health, Renton, WA, USA).

\subsubsection{Mechanism of Action}

OX40 is a type I transmembrane glycoprotein expressed on activated T cells; it is a member of the TNF receptor superfamily. In much the same fashion as CD40L, OX40 ligand (OX40L) is expressed at low levels in cells throughout the body under physiologic conditions and is upregulated in inflammatory conditions such as autoimmune processes [35]. The OX40/OX40L interaction involves several downstream signaling cascades that promote cell survival and enhance cytokine release from $T$ cells $[36,37]$. By leveraging these pathways, OX40 agonists have the potential to stimulate the immune response for use against cancer. 


\subsubsection{Indications}

There are no current FDA-approved indications for anti-OX40 antibodies. Several phase I/II studies assessing anti-OX40 therapy as monotherapy or combined therapy for solid malignancies are ongoing.

\section{Clinical Evidence}

\subsection{Overview}

Given the vast number of studies published in checkpoint modulation, a detailed account of each trial is beyond the scope of this review. Herein, we discuss the most clinically relevant studies, including those that led to FDA approval and/or changes in standards of care. More information about studies not covered in the text can be found in each class's respective table(s), alongside summaries of the studies discussed here.

\subsection{CTLA-4 Inhibitors}

\subsubsection{Ipilimumab}

\section{Overview}

Table A2 summarizes current clinical data for CTLA-4 inhibitors.

\section{Melanoma}

Ipilimumab was granted its first FDA approval in 2011 for unresectable/metastatic melanoma. This came about following completion of a phase III trial evaluating ipilimumab alone versus in combination with a gp100 cancer vaccine in patients with unresectable, refractory stage III or IV melanoma [38]. Numerous studies followed, exploring the role of ipilimumab both alone and in combination with other treatment modalities in varying stages of melanoma. Details of these investigations can be found in Table A2. Ipilimumab has since had its FDA approval expanded to include adjuvant treatment following complete surgical resection in patients with stage III disease.

\section{Renal Cell Carcinoma}

The phase III CheckMate 214 trial explored the efficacy of ipilimumab plus nivolumab for renal cell carcinoma (RCC), comparing it with first-line standard of care sunitinib. Results from this trial demonstrated significantly higher 18-month overall survival (OS) with combination ipilimumab/nivolumab compared with sunitinib (hazard ratio $(\mathrm{HR})=0.63, p<0.001$ ), as well as higher objective response rate (ORR) $(p<0.001)$ in the combination group [39]. Consequently, in April 2018, the FDA approved the use of combination ipilimumab/nivolumab for previously untreated, intermediateto poor-risk advanced RCC. Long-term follow up data (median follow up 32.4 months) published recently showed that in intermediate-risk or poor-risk patients, combination ipilimumab/nivolumab continued to be superior to sunitinib in terms of OS [40].

\section{Non-Small Cell Lung Cancer}

Encouraged by early clinical trials showing ipilimumab's activity against a variety of tumor types, researchers began investigating its use in patients with non-small cell lung cancer (NSCLC). However, ipilimumab alone or as part of combination therapy has not yielded meaningful clinical benefit and is not approved for lung cancer treatment [41,42].

\subsubsection{Tremelimumab}

While tremelimumab received orphan drug status for treatment of mesothelioma in 2015, it has yet to be FDA-approved for this indication. The DETERMINE study found no significant life prolongation 
in patients with previously treated malignant mesothelioma who were given tremelimumab, compared to those given placebo, with a median OS of 7.7 months in the treatment group and 7.3 months in the placebo group $(\mathrm{HR}=0.92, p=0.41)$ [43].

\subsection{PD-1 Inhibitors}

\subsubsection{Nivolumab}

\section{Overview}

Table A3 summarizes current clinical data for nivolumab.

\section{Bladder Cancer}

In 2017, nivolumab received FDA approval as second-line monotherapy for metastatic or surgically unresectable urothelial carcinoma that had progressed or recurred despite prior treatment with at least one platinum-based chemotherapy regimen. This was granted in response to a phase II clinical trial in which Sharma et al. treated 270 patients with metastatic urothelial carcinoma using a regimen of nivolumab $3 \mathrm{mg} / \mathrm{kg}$ IV every two weeks. ORR was 19.6\% across all PD-L1 expression subgroups, with $2 \%$ experiencing complete response (CR), and median progression-free survival (PFS) of 2.0 months. Grade 3 or 4 AEs occurred in $18 \%$ of patients treated, consisting mostly of grade 3 diarrhea and fatigue [44].

\section{Colorectal Cancer}

In 2017, Overman et al. published results from a phase II trial exploring the use of nivolumab monotherapy in patients with metastatic DNA mismatch repair-deficient (dMMR)/microsatellite instability-high (MSI-H) colorectal cancer (CRC). Patients included in the study had disease progression on, or after, at least one previous line of treatment, including a fluoropyrimidine and oxaliplatin or irinotecan. ORR was $31.1 \%$, with a median PFS of 14.3 months and 12-month PFS rate of $50 \%$. Grade 3 or 4 AEs were noted in $20 \%$ of patients [45]. These findings resulted in accelerated FDA approval in July 2017 for treatment of metastatic dMMR/MSI-H CRC that had progressed after treatment with the above chemotherapies.

\section{Head and Neck Squamous Cell Carcinoma}

In a randomized, open-label, phase III trial, Ferris et al. explored the use of nivolumab monotherapy in patients with recurrent or metastatic head and neck squamous cell carcinoma (HNSCC) that had progressed within six months after platinum-based chemotherapy. Patients were treated with either nivolumab or the investigator's choice of standard, single-agent systemic therapy (methotrexate, docetaxel or cetuximab). ORR was $13.3 \%$ in the nivolumab-treated patients $(2.50 \%$ complete response rate (CRR)), versus $5.8 \%$ in the patients treated with standard therapy $(0.83 \%$ CRR). Median OS was 7.5 months and 5.1 months in the nivolumab-treated versus standard treatment groups, respectively $(\mathrm{HR}=0.70, p=0.01)$. Fewer grade 3 or 4 adverse events $(\mathrm{AE})$ were reported in the nivolumab-treated group than in the standard chemotherapy group [46]. As a result of this study, nivolumab received FDA approval in November 2016 for recurrent HNSCC that had progressed on or after the above chemotherapies.

\section{Hepatocellular Carcinoma}

In a phase II open-label study, El-Khoueiry et al. explored the safety and efficacy of nivolumab monotherapy in the treatment of advanced hepatocellular carcinoma (HCC) with or without chronic viral hepatitis. Overall, ORR was $20 \%$ and median PFS was 4.0 months. The ORR was comparable regardless of previous sorafenib treatment. Grade 3 or 4 AEs were noted in 19\% of patients with no treatment-related deaths. Of note, PD-L1 expression did not appear to have a significant effect on 
response rates [47]. Based on these findings, nivolumab was granted FDA approval for treatment of HCC in patients who had failed vascular endothelial growth factor (VEGF) inhibition previously.

\section{Hodgkin Lymphoma}

Reed-Sternberg cells are known to utilize PD-L1 and PD-L2 to evade immune surveillance [48]. In response to promising results from a 2014 phase I study, a follow up phase II study was conducted that assessed the clinical benefit and safety of nivolumab in patients with Hodgkin lymphoma (HL) who had failed both autologous stem-cell transplantation (ASCT) and brentuximab vedotin [49]. ORR was $66.3 \%$ (9\% CRR) and median PFS was 10.0 months; grade 3 or 4 drug-related AEs occurred in $25 \%$ of patients, most commonly consisting of increased lipase and neutropenia [50]. These two studies were the bases of accelerated FDA approval of nivolumab in treatment of classical HL (cHL) with progression following ASCT and post-transplant brentuximab.

\section{Melanoma}

Based on the results of the phase III CheckMate 037 trial, nivolumab received its first FDA-approved indication in December 2014 for the treatment of unresectable/metastatic melanoma after failure of ipilimumab and, if BRAF V600 mutation positive, a BRAF inhibitor [51]. Like ipilimumab before it, several studies followed to investigate an expanded role for nivolumab in the management of melanoma. Current indications include combination therapy with ipilimumab for BRAF V600 wild-type, unresectable/metastatic melanoma and adjuvant therapy following complete surgical resection for patients with stage III melanoma. Details for each study can be found in Table A3.

\section{Non-Small Cell Lung Cancer}

The CheckMate 017 and CheckMate 057 phase III trials assessed nivolumab's role in treatment of advanced squamous NSCLC (sqNSCLC) and non-squamous NSCLC (NsqNSCLC), respectively [52,53]. In each, nivolumab monotherapy was compared to docetaxel following disease progression after one line of platinum-based chemotherapy. Median OS and ORR were higher in the nivolumab group in both trials, along with longer PFS for patients with advanced sqNSCLC. These studies were the bases of two FDA approvals in 2015 for the use of nivolumab in treatment of metastatic sqNSCLC and NsqNSCLC that had progressed following after platinum-based chemotherapy. Three year follow up data published in 2018 showed a continued, significant OS benefit compared to docetaxel in advanced NSCLC with $(\mathrm{HR}=0.68)$ or without $(\mathrm{HR}=0.70)$ liver metastases [54].

\section{Renal Cell Carcinoma}

In 2015, Motzer et al. reported the results of a randomized, open-label, phase III study comparing the effectiveness and safety of nivolumab monotherapy compared to everolimus in patients with RCC who had received previous treatment with one or two regimens of anti-angiogenic therapy. Between the nivolumab-treated and everolimus-treated groups, the median OS was 25.0 months versus 19.6 months, respectively ( $\mathrm{HR}=0.73, p=0.0018$ ). The ORR was $25 \%$ in the nivolumab group and $5 \%$ in the everolimus group $(p<0.001)$. Grade 3 or 4 AEs occurred in $19 \%$ of the patients treated with nivolumab and in $37 \%$ of patients treated with everolimus [55].

This study was followed by the CheckMate 214 trial, which compared combination ipilimumab/nivolumab to sunitinib for advanced RCC. These findings were discussed in the CTLA-4 section.

\section{Small Cell Lung Cancer}

In 2018, the FDA granted nivolumab accelerated approval for third-line treatment of metastatic small cell lung cancer (SCLC) based on the results of the CheckMate 032 trial, which compared nivolumab monotherapy to combination nivolumab and ipilimumab. For nivolumab monotherapy, 
an ORR of $10 \%$ was observed; median OS was 4.4 months. Grade 3 or 4 AEs occurred in $13 \%$ of patients. The three combination groups, each with varying doses, had ORR ranges from 19-33\%, with grade 3 or 4 toxicity rates of $19-30 \%$ [56].

\subsubsection{Pembrolizumab}

\section{Overview}

Table A4 summarizes current clinical data for pembrolizumab.

\section{Cervical Cancer}

Pembrolizumab received approval as a second-line treatment for cervical squamous cell carcinoma (CSCC) on the basis of KEYNOTE-158, a Phase $\mathrm{Ib}$ trial that enrolled 98 patients who had exhausted first-line therapy. Overall ORR was $12.2 \%$; all responses occurred among patients with PD-L1 positive tumors. In this subset of patients, ORR was $14.6 \%$. Median OS was 9.4 months in the total population and 11.0 months in those with PD-L1 positive tumors [57].

\section{Gastric Cancer}

On the basis of KEYNOTE-059, pembrolizumab received approval for recurrent, advanced gastric or gastroesophageal junction (GEJ) adenocarcinoma with progression on multiple prior therapies and known tumor PD-L1 expression. This phase II trial investigated the use of pembrolizumab in patients with gastric/GEJ adenocarcinoma previously treated with two or more systemic therapies including fluoropyrimidine and a platinum-based therapy, and a HER2/neu therapy if applicable. ORR was 15.5\% in the PD-L1 positive group, and $6.4 \%$ in the PD-L1 negative group. The median duration of response was 16.3 months in the PD-L1 positive group and 6.9 months in the PD-L1 negative group [58].

\section{Head and Neck Squamous Cell Carcinoma}

The KEYNOTE-012, KEYNOTE-040, and KEYNOTE-048 trials extensively evaluated pembrolizumab in the treatment of HNSCC, and several favorable outcomes were reported [59-61]. Current FDA-approved indications include first-line treatment for metastatic/unresectable recurrent HNSCC, both alone for tumors with known PD-L1 expression and in combination with platinum-based chemotherapy and fluorouracil for all patients. More information can be found in Table A4.

\section{Hepatocellular Carcinoma}

The KEYNOTE-224 phase II trial evaluated safety and efficacy of pembrolizumab in patients with HCC who had progressed or been intolerant to sorafenib. Results were published in June 2018 and showed an ORR of $17 \%$ with $44 \%$ of patients having stable disease, and a six-month OS rate of $77.9 \%$. Safety profile was found to be similar to that seen in previous studies of pembrolizumab [62]. This resulted in a new FDA-approved indication for treatment of HCC after failure of sorafenib.

\section{Hodgkin Lymphoma}

The known overexpression of PD-L1 and PD-L2 in HL was the basis of KEYNOTE-087, a phase II trial that evaluated pembrolizumab in three different cohorts stratified by prior treatment history [63]. Overall ORR was $69 \%$, with relatively equal distribution among cohorts. CRR was $22.4 \%$, and 31 patients had durable responses lasting six months or greater. This led to FDA approval of pembrolizumab for refractory or relapsed cHL after three or more treatments.

\section{Lung Cancer}

Pembrolizumab has been extensively studied in NSCLC and, more recently, SCLC in refractory and front-line settings. NSCLC represents one of the first FDA-approved indications granted to 
pembrolizumab, as it received accelerated approval in October 2015 for patients with metastatic NSCLC with known tumoral PD-L1 expression that had progressed on or after platinum-based chemotherapy [64]. It currently has indications as part of combination first-line treatment for metastatic NsqNSCLC and sqNSCLC, as well as first-line treatment for stage III disease in patients who are not candidates for surgical resection or definitive chemoradiation, whose tumors have no epidermal growth factor receptor (EGFR) or anaplastic lymphoma kinase (ALK) mutations, and whose tumors are known to express PD-L1. For SCLC, FDA approval was granted in June 2019 for patients with metastatic SCLC that had progressed on or after platinum-based chemotherapy and at least one other line of treatment. Please see Table A4 for further details of each study.

\section{Melanoma}

KEYNOTE-001, KEYNOTE-002, KEYNOTE-006, and KEYNOTE-054 assessed the role of pembrolizumab in management of melanoma in various settings [65-68]. On the basis of early results from KEYNOTE-001, pembrolizumab was granted its first ever FDA approval in September 2014. Indications have expanded since; they include metastatic melanoma with disease progression on ipilimumab and, if BRAF V600 mutation positive, a BRAF inhibitor, as well as adjuvant treatment following resection for stage III disease. Table A4 summarizes these studies.

\section{Merkel Cell Carcinoma}

The efficacy and safety of pembrolizumab in patients with systemic chemotherapy naïve advanced Merkel cell carcinoma (MCC) was assessed in the phase II KEYNOTE-017/Cancer Immunotherapy Trials Network-09 trial, which published final results earlier this year. Subjects received pembrolizumab monotherapy for up to two years, with an ORR of $56 \%$, CRR of $24 \%$ and partial response rate (PRR) of $32 \%$. Median PFS was 16.8 months, and two-year OS rate was $68.7 \%$. An association was noted between PD-L1 positive tumors and improved PFS and OS [69]. This study led to the approval of pembrolizumab as a first-line treatment in adult and pediatric locally advanced or metastatic disease.

\section{MSI-H or dMMR Tumors (Tissue-Agnostic)}

In 2017, the FDA approved pembrolizumab as the first tissue-agnostic cancer therapy for unresectable or metastatic solid cancers expressing MSI-H or dMMR, marking the first FDA approval based on biomarker expression rather than on specific disease. The approval was based off of the data from two main studies, KEYNOTE-016 and KEYNOTE-164, and post hoc analyses of three studies from which MSI-H or dMMR patients were identified: KEYNOTE-012, KEYNOTE-028, and KEYNOTE-158. The data consisted of 135 prospective patients and 14 retrospective patients whose MSI-H and dMMR status was identified using either polymerase chain reaction or immunohistochemistry. There were 90 CRC patients and 59 patients with one of 14 other solid tumor types who were treated with either pembrolizumab $200 \mathrm{mg}$ every three weeks or pembrolizumab $10 \mathrm{mg} / \mathrm{kg}$ every two weeks. The results showed an ORR of $39.6 \%$, with $78 \%$ of those patients showing a response duration greater than 6 months [70]. The approval currently exists for solid tumors that have progressed after treatment with no other current treatment options, or CRC that has progressed after being treated with fluoropyrimidine, oxaliplatin, and irinotecan.

\section{Primary Mediastinal B-Cell Lymphoma}

The phase I KEYNOTE-013 study [71] and follow-up phase II KEYNOTE-170 trial [71] evaluated pembrolizumab in patients with relapsed/refractory primary mediastinal B-cell lymphoma (rrPMBCL) who had failed, were ineligible for, or refused ASCT. Safety and efficacy data were promising in the phase I component, with similar response in the phase II follow up for patients with rrPMBCL that had relapsed after two or more lines of therapy. Phase II data demonstrated an ORR of $45 \%$ and CRR of $13 \%$; at the data cutoff, none of patients showing CR had relapsed. Median PFS was 5.5 months, median OS was not reached, and 12-month OS was 58\%. Neither trial was associated with unexpected 
or unacceptable toxicities [71]. These two studies resulted in FDA approval of pembrolizumab in treatment of $\operatorname{rrPMBCL}$ that had relapsed following two lines of chemotherapy.

\section{Renal Cell Carcinoma}

Following the success of a phase $\mathrm{Ib}$ investigation evaluating safety and efficacy of combination pembrolizumab/axitinib [72], the phase III KEYNOTE-426 compared combination pembrolizumab/axitinib to first-line sunitinib in patients with treatment-naïve advanced RCC. Results published earlier this year showed significantly longer OS in the pembrolizumab/axitinib group (HR $=0.53, p<0.0001$ ), as well as longer PFS (15.1 months vs. 11.1 months; HR $=0.69$, $p<0.001)$. ORR was also significantly higher in the combination group $(59.3 \%$ vs. $39.7 \% ; p<0.001)$ [73]. These results formed the basis of the recent FDA approval of pembrolizumab as first-line treatment in patients with advanced disease.

\subsection{PD-L1 Inhibitors}

\subsubsection{Atezolizumab}

\section{Overview}

Table A5 summarizes current clinical data for PD-L1 inhibitors.

\section{Bladder Cancer}

The IMvigor210 [74] and subsequent IMvigor211 [75] trials were the bases for initial FDA approval and expanded indications, respectively, of atezolizumab in treatment of urothelial cancer. IMvigor210 investigated atezolizumab monotherapy in patients with platinum-resistant, locally advanced or metastatic urothelial carcinoma; the follow up phase III IMvigor211 trial compared atezolizumab to various chemotherapies (investigator's choice of vinflunine, paclitaxel, or docetaxel) in a similar patient population. Phase II data from IMvigor210 showed superior ORR compared to historical chemotherapy ORR ( $15 \%$ vs. $10 \%$; stratified response rates to atezolizumab were as high as $26 \%$ in tumors with $>5 \%$ PD-L1-positive cells) [74], though this significant improvement in ORR was not reproduced on direct comparison of chemotherapy to atezolizumab in IMvigor211. Tolerability data in the phase III study strongly favored atezolizumab, however, as grade 3-4 AE rate in the atezolizumab group was $20 \%$ versus $43 \%$ in chemotherapy groups [75]. The current FDA-approved indications are for treatment of patients with locally advanced or metastatic disease who are (A) ineligible for cisplatin-based therapy and have biochemical evidence of $>5 \%$ PD-L1 expression within the tumor, or (B) ineligible for any platinum-based therapy.

\section{Breast Cancer (Triple-Negative)}

In 2018 Schmid et al. published findings from a phase III trial in which patients with therapy-naïve triple-negative breast cancer (TNBC) were treated with either combination atezolizumab/nab-paclitaxel or nab-paclitaxel plus placebo. Median PFS was found to be significantly longer in the atezolizumab plus nab-paclitaxel group compared with the placebo plus nab-paclitaxel group (7.2 months vs. 5.5 months, $\mathrm{HR}=0.80, p=0.002$ ); increased benefit was seen in subgroup analysis of those with baseline increased PD-L1 expression [76]. Thus, this combination was approved in March 2019 for patients with $>1 \%$ PD-L1 expression.

\section{Non-small Cell Lung Cancer}

In response to the POPLAR phase II trial, atezolizumab received FDA approval for treatment of metastatic NSCLC after progression on platinum-based chemotherapy. In this study, atezolizumab was compared to docetaxel in patients with previously treated advanced or metastatic NSCLC. Both OS $(\mathrm{HR}=0.73)$ and $\mathrm{AE}$ profiles were superior in the atezolizumab group [77]. 
A second indication for atezolizumab was granted in December 2018 as part of first-line combination therapy with bevacizumab, paclitaxel, and carboplatin for metastatic NsqNSCLC. This was in response to the phase III IMpower150 trial, in which bevacizumab plus carboplatin and paclitaxel (BCP) was compared to atezolizumab plus bevacizumab, carboplatin, and paclitaxel (ABCP) for patients with previously untreated metastatic NsqNSCLC. Median PFS and OS were significantly longer in the $\mathrm{ABCP}$ group compared to the $\mathrm{BCP}$ group (PFS: 8.3 months vs. 6.8 months; $\mathrm{HR}=0.62$, $p<0.001$; OS: 19.2 months vs. 14.7 months; $\mathrm{HR}=0.78, p=0.02$ ), and $\mathrm{ABCP}$ had comparable tolerability to that of each independent agent [78].

\section{Small Cell Lung Cancer}

In 2018, Horn et al. published results of the IMpower133 phase III trial comparing atezolizumab plus carboplatin and etoposide (ACE) to placebo plus carboplatin and etoposide (CE) in patients with extensive SCLC and no previous systemic therapy. Compared to CE, ACE had significantly longer OS (12.3 months versus 10.3 months; $\mathrm{HR}=0.70, p=0.0069$ ) and PFS (5.2 months vs. 4.3 months; $\mathrm{HR}=0.77$, $p=0.0170$ ), with a well-tolerated AE profile [79]. This led to an FDA approval in March 2019 as part of first-line combination treatment (ACE) in extensive-stage SCLC.

\subsubsection{Avelumab}

\section{Bladder Cancer}

Results from a phase Ib trial investigating the safety and efficacy of avelumab in patients with refractory metastatic urothelial carcinoma were published in 2017, demonstrating an ORR of $18.2 \%$, with a CRR of $11.2 \%$ and 12 -month OS rate of $54.3 \%$ [80]. This data supported avelumab's excellent efficacy in advanced bladder cancer, leading to accelerated FDA approval in May 2017 for treatment of metastatic urothelial carcinoma refractory to 12 months of platinum-based chemotherapy.

\section{Merkel Cell Carcinoma}

Avelumab was granted its first FDA approval in March 2017 for the treatment of metastatic MCC in patients 12 years and older. This was in response to the findings from the JAVELIN Merkel 200 phase II trial evaluating avelumab monotherapy in patients with stage IV, chemotherapy-resistant, MCC. In this study, avelumab was associated with an ORR of $31.8 \%$ and a $9 \%$ CRR, as well as a favorable safety profile [81].

\section{Renal Cell Carcinoma}

In a recent phase III trial, avelumab in combination with axitinib was compared to sunitinib monotherapy for first-line treatment of RCC. A clinically significant increase in PFS (HR $=0.61$, $p<0.001$ ) and ORR was demonstrated in the combination avelumab/axitinib group compared to the sunitinib group. In response to these results, this combination was approved for first-line treatment in patients with advanced RCC in May 2019 [82].

\subsubsection{Durvalumab}

\section{Bladder Cancer}

Durvalumab was granted accelerated FDA approval in May 2017 for the treatment of patients with locally advanced or metastatic urothelial carcinoma that had progressed during or following platinum-based chemotherapy and/or within 12 months of neoadjuvant or adjuvant treatment with platinum-based chemotherapy. This approval followed the results from a phase I/II study published by Massard et al. which demonstrated an ORR of 31\% in patients with metastatic bladder cancer who had progressed on, been ineligible for, or refused any number of prior therapies [83]. 
Non-Small Cell Lung Cancer

In 2017, Antonia et al. published results from the PACIFIC phase III trial comparing durvalumab to placebo as consolidation therapy in patients with stage III NSCLC that did not progress after two or more cycles of platinum-based chemoradiotherapy. Median PFS was significantly longer in the durvalumab group compared to placebo (16.8 months vs. 5.6 months; $\mathrm{HR}=0.52, p<0.001$ ), and AE profile was acceptable [84]. This led to FDA approval in February 2018 for consolidation therapy in patients with unresectable stage III NSCLC without progression during concurrent platinum-based chemotherapy.

\subsection{LAG-3 Inhibitors}

\subsubsection{Relatlimab}

In 2017, Ascierto et al. published preliminary phase I/IIa data from patients treated with combination relatlimab/nivolumab for melanoma that had progressed on prior anti-PD-1/anti-PD-L1 therapy. ORR for all patients was $11.5 \%$ ( $1 \mathrm{CR}, 6 \mathrm{PR})$, with subgroup analyses demonstrating an ORR of $18 \%$ in patients with tumoral LAG-3 expression $\geq 1 \%$ [24]. Early data from a separate phase I study (NCT02658981) investigating relatlimab monotherapy and combination nivolumab/relatlimab for recurrent glioblastoma multiforme (GBM) showed excellent tolerability of relatlimab alone, with no dose-limiting toxicities (DLT) reported at the maximum planned dose [85].

\subsubsection{Ongoing Studies}

Several clinical trials are underway investigating the role of anti-LAG-3 therapies in various malignancies. These are presented in Table A6.

\subsection{TIM-3 Inhibitors}

As of September 2019, no clinical data has been published for TIM-3 inhibitors. Ongoing studies are summarized in Table A6.

\subsection{CD40 Agonists}

\subsubsection{APX0050M}

\section{Melanoma}

APX005M in combination with nivolumab is under current exploration in the treatment of metastatic melanoma. New clinical data was recently published from a phase $\mathrm{Ib}$ dose-escalation/phase II dose-expansion trial investigating this combination in patients with metastatic melanoma that had progressed on anti-PD-1 monotherapy. A good safety profile was reported; phase II data showed partial response in two of 12 subjects, along with stable disease in three subjects [86].

\section{Pancreatic Cancer}

Early results from an ongoing phase Ib study investigating APX005M in combination with chemotherapy (gemcitabine and nab-paclitaxel) and nivolumab for previously untreated metastatic pancreatic ductal adenocarcinoma were published in July 2019, with evidence of tolerable AE profiles and favorable anti-tumor activity of APX005M at varying doses and combinations [87]. A randomized phase II is in the works to expand upon the groundwork laid by this study.

\subsubsection{CP-870,893}

\section{Metastatic Melanoma}

In 2015, Bajor et al. published the results of a phase I dose escalation study investigating combination CP-870,893/tremelimumab in patients with metastatic melanoma. A total of 24 patients 
were enrolled; four different dose combinations were employed. Three DLTs were reported. The maximum tolerated doses (MTD) were found to be $0.2 \mathrm{mg} / \mathrm{kg}$ of CP-870,893 and $10 \mathrm{mg} / \mathrm{kg}$ of tremelimumab. ORR was $27.3 \%$, CRR was $9.1 \%$, and PRR was $18.2 \%$. Median PFS was 22 months and median OS was 26.1 months [88].

\section{Pancreatic Cancer}

In November 2013, Beatty et al. published results of a phase I study, which evaluated the MTD, safety profile, and efficacy of combination CP-870,893/gemcitabine for the treatment of advanced pancreatic ductal carcinoma. This combination was tolerated well, with only one DLT. Four patients achieved partial response to treatment [89].

\subsubsection{Ongoing Studies}

Other ongoing clinical studies evaluating CD40 agonists are summarized in Table A7.

\subsection{OX40 Agonists}

\subsection{1. $9 \mathrm{~B} 12$}

Advanced Solid Malignancies

Safety and efficacy data for 9B12 was published for patients with various metastatic solid malignancies refractory to conventional chemotherapy [90-92]. The safety profile was acceptable, and regression of at least one tumor nodule was seen in 12 of 30 patients $(40 \%)$.

\subsubsection{Ongoing Studies}

Current investigations in OX40 agonist therapies are highlighted in Table A7.

\subsection{Combination Therapies}

As the research, development, and approval of checkpoint modulators has expanded, combination therapy, both with other checkpoint inhibitors as well as with traditional chemotherapy and radiotherapy, has garnered much therapeutic interest. Many of these combinations have been discussed in the sections above. As of September 2019, there are a total of 2250 active trials investigating the use of anti-PD-1/PD-L1 mAbs in various diseases, and of those, 1716 are testing combination therapies with other antineoplastic agents [93].

Combination therapies are studied in first-line settings with the intention of improving response rates to existing PD-1/PD-L1 monotherapy and to prevent activation of resistance pathways, ensuring durability of responses. The first combination therapy to be approved by the FDA was that of ipilimumab and nivolumab in 2015 for BRAF V600 wild type melanoma. This combination also gained approval for use in RCC and MSI-H CRC. The second combination to receive FDA approval was that of pembrolizumab and conventional chemotherapy in May 2018 for use in NsqNSCLC. Soon after, in October 2018, this combination gained approval in treating sqNSCLC and then again in June 2019 for treatment of patients with HNSCC. Additionally, pembrolizumab and avelumab have been approved in combination with the tyrosine kinase inhibitor axitinib for first-line treatment of advanced RCC. Most recently, the combination of pembrolizumab and the angiogenesis inhibitor lenvatinib (Lenvima, Eisai Co.) was granted approval in September 2019 for use in advanced endometrial carcinoma that has progressed on prior systemic therapy, is not MSI-H or dMMR, and is not amenable to curative surgery or radiation [94].

Combination therapies are also being evaluated in the PD-1/PD-L1-refractory setting as illustrated above, with a few combinations (PD-1 mAb + CD40 agonist, PD-1 mAb + LAG-3 inhibitor) showing promising initial data in terms of ORR and disease control rates [24,86,88]. Further long-term data from these studies would be extremely helpful, as PD-1/PD-L1 mAb resistance is a major therapeutic 
concern now. Some of these combinations (PD-1 antibody + LAG-3 inhibitor) are even being evaluated in the first-line setting based on the preliminary data noted in refractory setting (Trial NCT03743766).

\section{Immunotherapy Resistance and Its Implications}

Despite the success of checkpoint immunomodulation, not all populations benefit. Resistance is well-documented, acting as a barrier to a durable (and/or early) tumor response [95-98].

Resistance is categorized into two classifications: primary and secondary. Primary resistance is defined by absent tumor response to initial therapy. This is in contrast to secondary resistance, in which originally susceptible tumor cells adapt over time to immunotherapy. Mechanisms of resistance are numerous; both tumor-cell-intrinsic and tumor-cell-extrinsic sources have been described. Similar methods are demonstrated in both primary and secondary resistance. Examples of tumor-cell-intrinsic mechanisms include lack of neoantigen development, impaired antigen processing and presentation, altered intracellular signaling pathways, and upregulated or constitutive expression of inhibitory ligands (Figure 6) [49,99-104]. Tumor-cell-extrinsic mechanisms include increased recruitment and activity of inhibitory immune cells within the TME and upregulation of LAG-3, TIM-3, and other inhibitory ligands (Figure 7) [27,30,97,105-107].

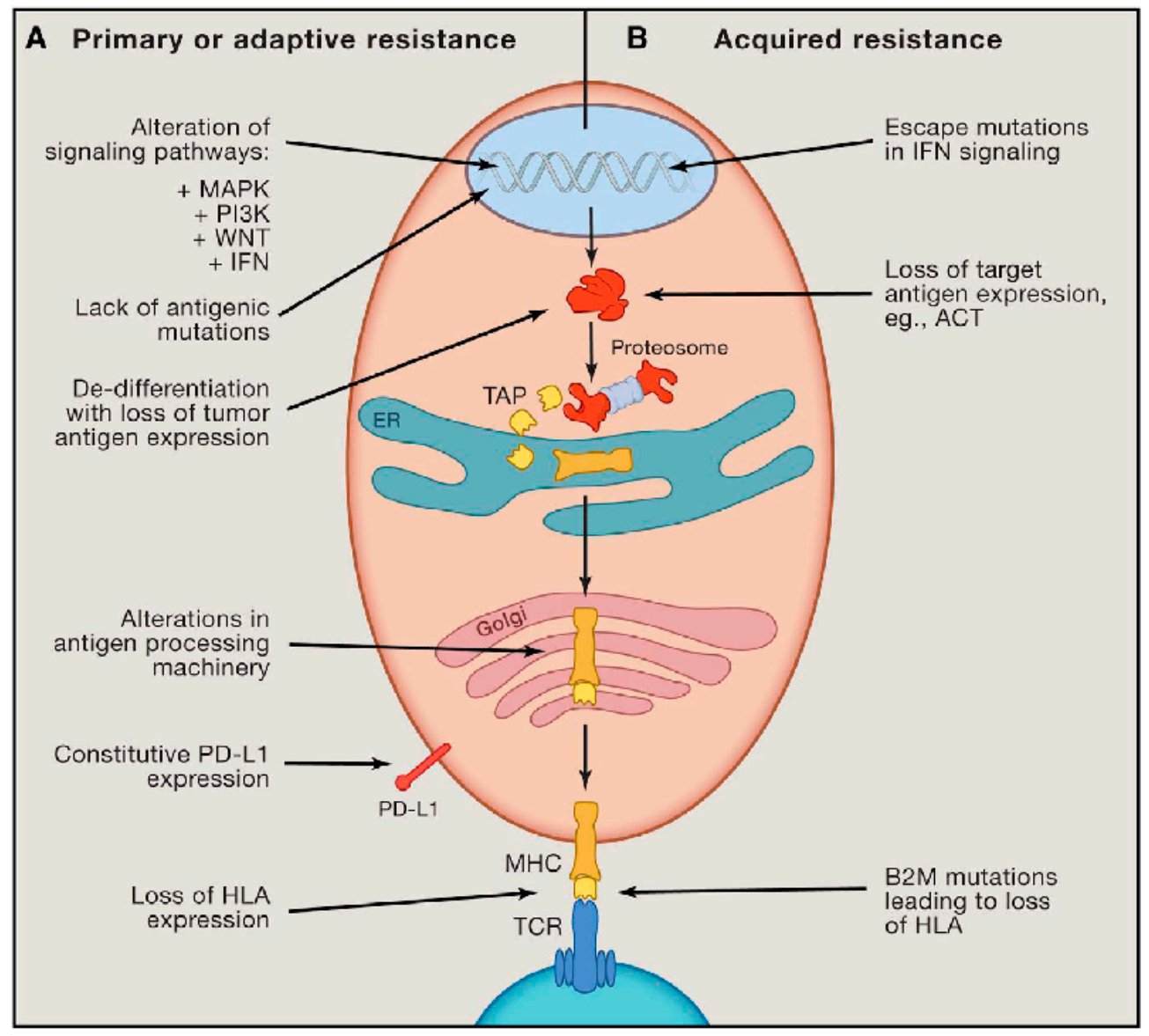

Figure 6. Intrinsic mechanisms of resistance to immunotherapy. Description: (A) Examples of intrinsic mechanisms of adaptive resistance, including altered signaling pathways, limited mutational burden, de-differentiation of tumor resulting in a loss of neoantigen expression, defective antigen processing, constitutive PD-L1 expression, and loss of HLA expression. (B) Examples of intrinsic mechanisms of acquired resistance, including loss of antigenic target, loss of HLA expression, and escape mutations in IFN signaling [97]. 


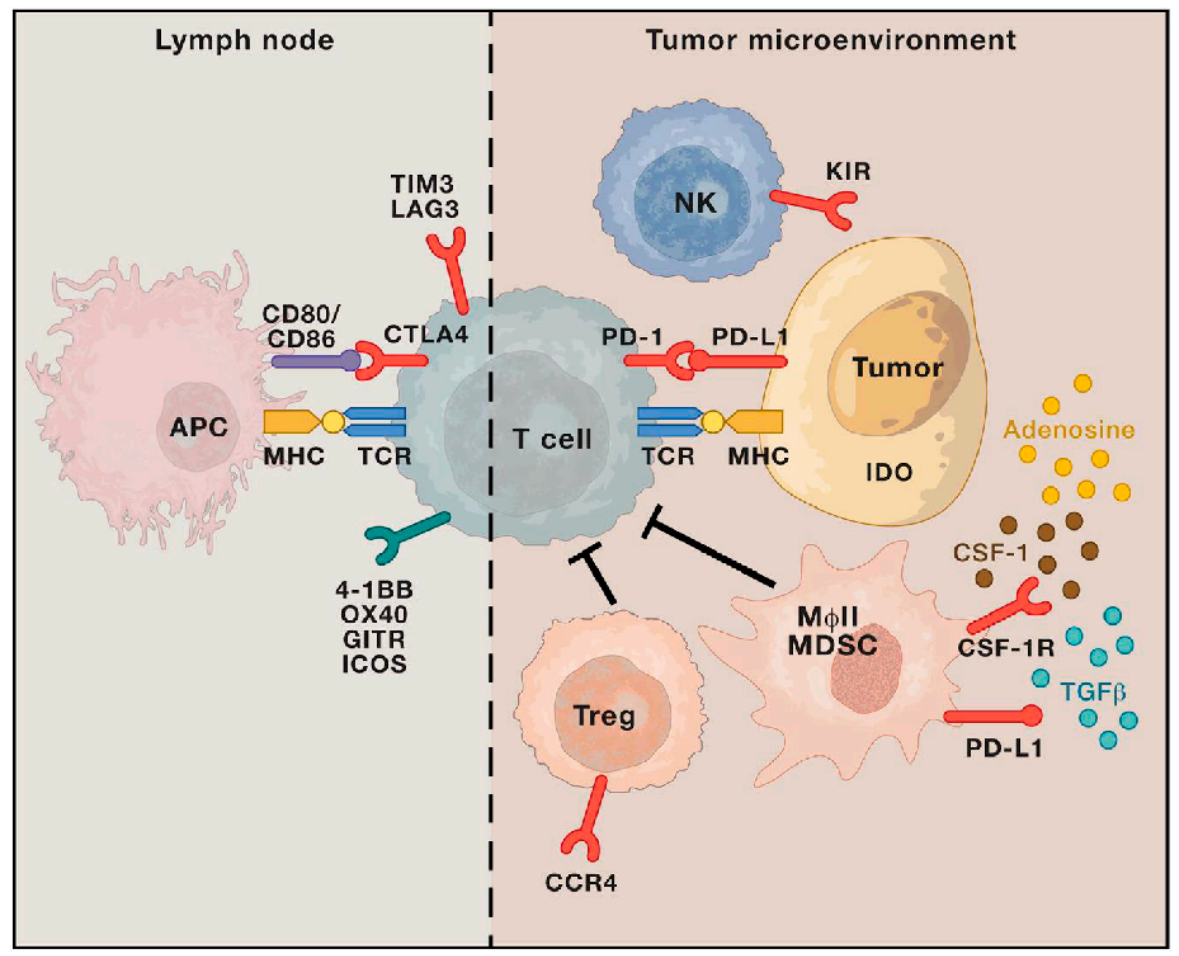

Figure 7. Extrinsic mechanisms of resistance to immunotherapy. Description: Examples of extrinsic mechanisms of resistance, including upregulated or constitutive immune checkpoint expression, immunosuppressive cytokine release (CSF-1, TGF $\beta$, adenosine) within the tumor microenvironment, $\mathrm{T}$ cell exhaustion and phenotypic alteration, and increased immunosuppressive cell populations (Treg, MDSC, MфII) [97].

Several strategies are currently being researched to combat resistance to immunotherapy, including combination therapy with other checkpoint inhibitors, chemotherapy and/or radiation. This has been discussed above in Section 3.9. Generally, a single immune checkpoint inhibitor-most frequently an anti-PD-1 mAb—is employed with one or more additional therapies [97,98]. This framework is evident in many of the studies discussed previously, with a number of FDA approvals granted in response to the superior tumoral response and/or survival rates. Investigatory studies have shown promise for combination anti-PD-1 + anti-LAG-3 and anti-PD-1 + CD40 agonist therapies in patients with known PD-1/PD-L1-refractory disease states. In general, response rates tend to improve with combination therapy, but tolerability and AE profile worsens. Thus, a careful assessment of clinical benefit versus toxicity is necessary when evaluating the utility of combination therapies in different populations.

The use of oncolytic viruses in conjunction with immunotherapy is an additional emerging strategy to fight resistance. Oncolytic viruses are genetically engineered viral strains designed to invade and lyse malignant cells without harming normal cells [108]. Furthermore, when injected locally, oncolytic viruses can change immune-secluded "cold" tumors to immune-rich "hot" tumors, which helps immunotherapy work more effectively. The first FDA-approved oncolytic virus, talimogene laherparepvec, was shown to be of benefit in the treatment of recurrent, unresectable stage IIIB-IVM1a melanoma [109]. Combination with immune checkpoint inhibitors has subsequently been explored. Thus far, early clinical data in advanced melanoma patients appear promising [110]. Investigations in treatment of other malignancies are ongoing.

Furthermore, targeting specific components of the TME is another topic undergoing extensive investigation. Stromal cells within the TME have been implicated in multiple pro-neoplastic processes, including physical support, selective promotion of tumorigenesis, angiogenesis, tissue remodeling, and suppression of anti-tumor immunity [111-113]. Examples include myeloid-derived cell populations and cancer-associated fibroblasts (CAF). 
Somewhat paradoxically, infiltration of the TME by various myeloid lineages has been associated with inhibited anti-tumor immunity, pro-tumor effects, and poorer overall prognoses [111]. Most commonly implicated cells include tumor-associated macrophages, myeloid-derived suppressor cells, tumor-associated dendritic cells, and tumor-associated neutrophils. The pro-malignancy effects are thought to be partially due to the plastic nature of the myeloid lineages; in response to various elements of the TME, including hypoxia, endoplasmic reticulum stress, and local immunosuppressive cytokines, these immune cells become polarized into immunotolerant phenotypes (M2, N2, et cetera) [114-117]. This, in turn, upregulates the expression of pro-angiogenic genes (VEGF), increases immunosuppressive and tissue remodeling cytokines (IL-10, transforming growth factor-beta (TGF- $\beta$ )), suppresses pre-activated T cell proliferation, enhances recruitment and proliferation of Tregs and other immunotolerant myeloid lineages (macrophage colony-stimulating factor (M-CSF), CCL2), promotes Th2 differentiation while suppressing Th1 responses (PGE2), and decreases cancer neo-antigen expression by APCs [118-123]. Understandably, the prospect of limiting these effects is appealing in cancer therapy, and depletion of myeloid cells has been shown in mice to correlate to decreased tumor growth [113]. Broadly, two strategies have been investigated in pre-clinical models: depletion of myeloid cell numbers (via blockade of CCL2/CCR2, M-CSF/M-CSF receptor, and VEGF/VEGF receptor) and manipulation of myeloid cell function/plasticity (introduction of anti-cancer cytokines to TME, inhibition of STAT3 signaling, triggering toll-like receptors 3 and 9, and inhibiting CD36, inducible nitric oxide synthase, arginase- 1 , and indoleamine 2,3 deoxygenase) [113]. Results thus far have shown therapeutic benefit with both approaches; however, more research is necessary at this time to determine optimal targets. Addressing the problem of myeloid cells may prove to be helpful in reducing checkpoint modulator resistance as these potential therapies transition from the pre-clinical to clinical environment in the coming years.

Similarly, CAFs represent other troublesome inhabitants of the TME; they are also associated with significant immunosuppressive effects. A fairly heterogenous group with varying traits, CAFs typically have high metabolic activity, owing to their increased synthetic capacity compared to the traditionally indolent "normal" fibroblasts [112]. Many types of CAFs produce multiple growth factors and cytokines, including TGF- $\beta$, VEGF, and IL-6, which assist in immune evasion [124]. Some CAFs are also known for their extensive production of extracellular matrix, which blocks access of immune cells to the tumor. Therapies directed at CAFs thus far are primarily in pre-clinical stages, though some early clinical studies are ongoing. Mechanisms explored include inhibition of CAF function (TGF- $\beta$ inhibitors, Hedgehog inhibitors, CXCR4 inhibitors), reprogramming CAFs to normal fibroblasts (vitamin A, D), and depletion of stromal extracellular matrix (anti-tenascin inhibitor). For certain CAF subtypes (fibroblast activation protein+), direct elimination via transgenic techniques and oncolytic viruses is also under investigation [112]. Early findings are promising, but additional research is necessary to further the understanding of these problematic TME inhabitants as a means to better select the most appropriate targets in this context.

\section{Conclusions}

Immune checkpoint modulators have garnered significant attention in the management of solid malignancies over the past several years mainly due to fewer side effects than chemotherapy, as well as their ability to result in durable responses in certain patients. The advent of these therapies has significantly changed treatment paradigms and prognoses of several cancers. Given these many advances, more and more immunomodulatory therapies are being explored, and those with pre-established indications are undergoing further investigations to expand their footprint within the oncologist's armamentarium. Nonetheless, many patients do not respond to immunotherapy, and resistance proves challenging. As we explore strategies to combat resistance to immunotherapies, continued research to identify biomarkers to predict response and side effects of immunotherapy is crucial. 
Author Contributions: N.G.: reviewed publications, contributed to first manuscript, revised manuscripts, co-authored final manuscript. K.G.-G.: reviewed publications, contributed to first manuscript, revised manuscripts, co-authored final manuscript. P.M.: reviewed publications, contributed to first manuscript, reviewed final manuscript. Y.G.: reviewed publications, contributed to first manuscript, reviewed final manuscript. M.B.: reviewed publications, contributed to first manuscript, reviewed final manuscript. C.M.: reviewed publications, contributed to first manuscript, reviewed final manuscript. S.S.: conceived of review, reviewed publications, co-authored final manuscript.

Funding: No funding sources outside of our institution were utilized.

Conflicts of Interest: The authors declare no conflict of interest.

\section{Abbreviations}

\begin{tabular}{|c|c|}
\hline Abbreviation & Interpretation \\
\hline $\mathrm{AE}$ & adverse event \\
\hline ALK & anaplastic lymphoma kinase \\
\hline $\mathrm{APC}$ & antigen-presenting cell \\
\hline ASCT & autologous stem-cell transplantation \\
\hline B2M & beta-2-microglobulin \\
\hline BTLA & B and T lymphocyte attenuator \\
\hline CAF & cancer-associated fibroblast \\
\hline CCR4 & C-C chemokine receptor type 4 \\
\hline CD & cluster of differentiation \\
\hline CD40L & CD40 ligand \\
\hline cHL & classical Hodgkin lymphoma \\
\hline $\mathrm{CR}$ & complete response \\
\hline CRC & colorectal cancer \\
\hline CRR & complete response rate \\
\hline $\mathrm{CSCC}$ & cervical squamous cell carcinoma \\
\hline CSF-1 & colony stimulating factor 1 \\
\hline CTLA-4 & cytotoxic T-lymphocyte-associated protein 4 \\
\hline DLT & dose-limiting toxicity \\
\hline dMMR & DNA mismatch repair-deficient \\
\hline EGFR & epidermal growth factor receptor \\
\hline ER & endoplasmic reticulum \\
\hline FDA & Food and Drug Administration \\
\hline GAL9 & galectin-9 \\
\hline GBM & glioblastoma multiforme \\
\hline GEJ & gastroesophageal junction \\
\hline GITR & glucocorticoid-induced tumor necrosis factor receptor \\
\hline $\mathrm{HCC}$ & hepatocellular carcinoma \\
\hline $\mathrm{HL}$ & Hodgkin lymphoma \\
\hline HLA & human leukocyte antigen \\
\hline HR & hazard ratio \\
\hline HVEM & tumor necrosis factor receptor superfamily member 14 \\
\hline ICOS & inducible T-cell co-stimulator \\
\hline IDO & indoleamine 2,3-dioxygenase \\
\hline IFN & interferon \\
\hline IL & interleukin \\
\hline KIR & killer cell immunoglobulin-like receptors \\
\hline LAG-3 & lymphocyte-activation gene-3 \\
\hline $\mathrm{mAb}$ & monoclonal antibody \\
\hline
\end{tabular}




$\begin{array}{ll}\text { Abbreviation } & \text { Interpretation } \\ \text { MCC } & \text { Merkel cell carcinoma } \\ \text { M-CSF } & \text { macrophage colony-stimulating factor } \\ \text { MDSC } & \text { myeloid-derived suppressor cell } \\ \text { MHC } & \text { major histocompatibility complex } \\ \text { MSI-H } & \text { microsatellite instability-high } \\ \text { MTD } & \text { maximum tolerated dose } \\ \text { M } \phi I I & \text { type II macrophage } \\ \text { NK } & \text { natural killer } \\ \text { NSCLC } & \text { non-small cell lung cancer } \\ \text { NsqNSCLC } & \text { non-squamous non-small cell lung cancer } \\ \text { ORR } & \text { objective response rate } \\ \text { OS } & \text { overall survival } \\ \text { OX40L } & \text { OX40 ligand } \\ \text { PD-1 } & \text { programmed cell death protein-1 } \\ \text { PD-L1 } & \text { programmed death-ligand 1 } \\ \text { PD-L2 } & \text { programmed death-ligand 2 } \\ \text { PFS } & \text { progression-free survival } \\ \text { PI3K } & \text { phosphatidyl-inositol 3-kinase } \\ \text { PRR } & \text { partial response rate } \\ \text { RCC } & \text { renal cell carcinoma } \\ \text { rrPMBCL } & \text { relapsed/refractory primary mediastinal B-cell lymphoma } \\ \text { SCLC } & \text { small cell lung cancer } \\ \text { sqNSCLC } & \text { squamous non-small cell lung cancer } \\ \text { TAP } & \text { transporter associated with antigen processing } \\ \text { TCR } & \text { T cell receptor } \\ \text { TGF- } \beta & \text { transforming growth factor-beta } \\ \text { TIM-3 } & \text { T cell immunoglobulin and mucin-domain containing-3 } \\ \text { TME } & \text { tumor microenvironment } \\ \text { TNBC } & \text { triple-negative breast cancer } \\ \text { TNF } & \text { tumor necrosis factor } \\ \text { Treg } & \text { regulatory T cell } \\ \text { VEGF } & \text { vascular endothelial growth factor } \\ & \end{array}$




\section{Appendix A}

Table A1. Summary of immune checkpoint modulator classes and indications.

\begin{tabular}{|c|c|c|c|c|c|c|c|c|}
\hline Target & mAb Function & Drug Examples & IgG Class & Epitope & $\begin{array}{l}\text { Affinity } \\
\text { (Human } \\
\text { Targets) }\end{array}$ & $\begin{array}{l}\text { Year of First } \\
\text { FDA Approval }\end{array}$ & $\begin{array}{l}\text { FDA-Approved } \\
\text { Indications }\end{array}$ & $\begin{array}{c}\text { Examples of Indications } \\
\text { with Clinical Data but } \\
\text { no FDA Approval }\end{array}$ \\
\hline CTLA-4 & Antagonist & $\begin{array}{c}\text { Ipilimumab } \\
\text { (Bristol-Myers Squibb), } \\
\text { tremelimumab } \\
\text { (MedImmune/AstraZeneca) }\end{array}$ & $\begin{array}{l}\text { I: IgG1 } \\
\text { T: IgG2 }\end{array}$ & $\begin{array}{l}\text { I: CTLA-4 F, G } \\
\text { strands; TBS } \\
1709 \AA^{2}[4] \\
\text { T: CTLA-4 F, G } \\
\text { stands; TBS 1802 } \\
\AA^{2}[4,21]\end{array}$ & $\begin{array}{l}\text { I: } \mathrm{K}_{\mathrm{d}}=18.2 \mathrm{nM} \\
(\mathrm{CTLA}-4)[4,21] \\
\text { T: } \mathrm{K}_{\mathrm{d}}=5.9 \mathrm{nM} \\
(\text { CTLA-4) }[4,21]\end{array}$ & 2011 & $\begin{array}{c}\text { I: Melanoma, RCC, CRC } \\
\text { T: }-\end{array}$ & $\begin{array}{l}\text { I: NSCLC, Prostate } \\
\text { cancer, SCLC } \\
\text { T: Melanoma, } \\
\text { mesothelioma, NSCLC }\end{array}$ \\
\hline PD-1 & Antagonist & $\begin{array}{c}\text { Nivolumab } \\
\text { (Bristol-Myers Squibb), } \\
\text { pembrolizumab } \\
\text { (Merck \& Co) }\end{array}$ & $\begin{array}{l}\text { N: IgG4 } \\
\text { P: IgG4 }\end{array}$ & $\begin{array}{l}\text { N: PD-1 N-loop; } \\
\text { TBS 1487 } \AA^{2}[21] \\
\text { P: PD-1 CD loop; } \\
\text { TBS 2126 } \AA^{2}[21]\end{array}$ & $\begin{array}{c}\mathrm{N}: \mathrm{K}_{\mathrm{d}}=2.6-3.1 \\
\mathrm{nM} \\
(\mathrm{PD}-1)[125,126] \\
\mathrm{P}: \mathrm{K}_{\mathrm{d}}=29.0 \mathrm{pM} \\
(\mathrm{PD}-1)[125,126]\end{array}$ & 2014 & $\begin{array}{c}\text { N: Bladder cancer, CRC, } \\
\text { HCC, HL, HNSCC, } \\
\text { melanoma, NSCLC, RCC, } \\
\text { SCLC } \\
\text { P: Bladder cancer, CSCC, } \\
\text { ESCC, gastric or GEJ } \\
\text { adenocarcinoma, HCC, } \\
\text { HL, HNSCC, MCC, } \\
\text { melanoma, } \\
\text { MSI-H/dMMR tumors } \\
\text { (tissue-agnostic), NSCLC, } \\
\text { PMBCL, RCC, SCLC, } \\
\text { endometrial carcinoma }\end{array}$ & $\begin{array}{l}\text { N: Anal cancer, GBM, } \\
\text { Gastric/GEJ cancer, } \\
\text { mesothelioma } \\
\text { P: Nasopharyngeal } \\
\text { carcinoma }\end{array}$ \\
\hline PD-L1 & Antagonist & $\begin{array}{c}\text { Atezolizumab } \\
\text { (Roche-Genentech), } \\
\text { avelumab (Merck/Pfizer), } \\
\text { durvalumab } \\
\text { MedImmune/AstraZeneca }\end{array}$ & $\begin{array}{l}\text { At: IgG1 } \\
\text { Av: IgG1 } \\
\text { D: IgG1 }\end{array}$ & $\begin{array}{c}\text { At: PD-L1 } \\
\text { CC'FG } \beta \text {-sheet; } \\
\text { TBS } 1970 \AA^{2}[23] \\
\text { Av: PD-L1 } \\
\text { CC'FG } \beta \text {-sheet } \\
\text { and N-terminus; } \\
\text { TBS } 1865 \AA^{2}[23] \\
\text { D: PD-L1 } \\
\text { CC'FG } \beta \text {-sheet } \\
\text { and N-terminus; } \\
\text { TBS } 2106 \AA^{2}[23]\end{array}$ & $\begin{array}{l}\text { At: } \mathrm{K}_{\mathrm{d}}=0.4 \mathrm{nM} \\
\quad(\mathrm{PD}-\mathrm{L} 1)[23] \\
\text { Av: } \mathrm{K}_{\mathrm{d}}=42.1 \\
\text { pM (PD-L1) [23] } \\
\text { D: } \mathrm{K}_{\mathrm{d}}=22.0 \mathrm{pM} \\
(\mathrm{PD}-\mathrm{L} 1)[23]\end{array}$ & 2016 & $\begin{array}{c}\text { At: Bladder cancer, } \\
\text { NSCLC, SCLC, TNBC } \\
\text { Av: Bladder cancer, MCC, } \\
\text { RCC } \\
\text { D: Bladder cancer, } \\
\text { NSCLC }\end{array}$ & $\begin{array}{l}\text { At: Melanoma } \\
\text { Av: NSCLC } \\
\text { D: Mesothelioma }\end{array}$ \\
\hline
\end{tabular}


Table A2. Clinical data for CTLA-4 inhibitors.

\begin{tabular}{|c|c|c|c|c|c|c|c|c|c|c|c|c|}
\hline Therapy & Cancer & Study & Phase & Stage of Disease & Treatment Regimen & $\mathbf{N}$ & $\begin{array}{l}\text { Median OS } \\
\text { (Months) }\end{array}$ & $\begin{array}{l}\text { Median PFS } \\
\text { (Months) }\end{array}$ & ORR (\%) & CRR (\%) & $\begin{array}{c}\text { Grade } \\
\text { III/IV } \\
\text { AEs (\%) }\end{array}$ & $\begin{array}{l}\text { Resultant FDA-Approved } \\
\text { Indication }\end{array}$ \\
\hline \multirow[t]{5}{*}{ Ipilimumab } & CRC & $\begin{array}{l}\text { Overman et al. } \\
\text { (2018) [127] } \\
\text { CheckMate } 142\end{array}$ & II & $\begin{array}{l}\text { Recurrent of } \\
\text { metastatic } \\
\text { dMMR/MSI-H } \\
\text { disease }\end{array}$ & $\begin{array}{c}\text { Nivolumab } 3 \mathrm{mg} / \mathrm{kg}+ \\
\text { ipilimumab } 1 \mathrm{mg} / \mathrm{kg} \\
\text { Q3wks x4 followed by } \\
\text { nivolumab } 3 \mathrm{mg} / \mathrm{kg} \\
\text { Q2wks }\end{array}$ & 119 & NR & NR & $\begin{array}{l}49 \text { (95\% CI: } \\
39.5-58.1)\end{array}$ & 4 & 32.0 & $\begin{array}{l}\text { Approved in combination } \\
\text { with nivolumab for } \\
\text { previously treated } \\
\text { MSI-H/dMMR metastatic } \\
\text { CRC (July 2018) }\end{array}$ \\
\hline & \multirow[t]{3}{*}{ Melanoma } & $\begin{array}{l}\text { Hodi et al. } \\
\text { (2010) [38] }\end{array}$ & III & $\begin{array}{l}\text { Unresectable, stage } \\
\text { III/IV disease }\end{array}$ & $\begin{array}{l}\text { A. Ipilimumab } 3 \mathrm{mg} / \mathrm{kg}+ \\
\text { gp100 peptide vaccine } \\
\text { B. Ipilimumab } 3 \mathrm{mg} / \mathrm{kg} \\
\text { monotherapy } \\
\text { C. gp100 peptide vaccine } \\
\text { monotherapy } \\
\text { (all Q3wks.) }\end{array}$ & $\begin{aligned} 676 \\
\mathbf{A}=403 \\
\mathbf{B}=137 \\
\mathbf{C}=136\end{aligned}$ & $\begin{array}{c}\text { A. } 10.0 \text { ( } 95 \% \text { CI: } \\
8.5-11.5) \\
\text { B. } 10.1(95 \% \text { CI: } \\
8.0-13.8) \\
\text { C. } 6.4(95 \% \mathrm{CI}: \\
5.5-8.7) \\
\text { (HR }=0.68, \\
p<0.001 \text { A vs. C; } \\
\text { HR }=0.66, \\
p=0.003 \text { B vs. C) }\end{array}$ & - & $\begin{array}{c}\text { A. } 5.7(95 \% \text { CI: } \\
3.7-8.4) \\
\text { B. } 10.9(95 \% \text { CI: } \\
\text { 6.3-17.4) } \\
\text { C. } 1.5 \\
(p=0.04 \text { A vs. B; } \\
p=0.04 \text { vs. C; } \\
p=0.001 \text { B vs. C) }\end{array}$ & $\begin{array}{l}\text { A. } 0.2 \\
\text { B. } 1.5 \\
\text { C. } 0\end{array}$ & $\begin{array}{l}\text { A.+ } \\
\text { B. } 10-15 \\
\text { C. } 3.0\end{array}$ & $\begin{array}{l}\text { Unresectable or metastatic } \\
\text { melanoma (March 2011) }\end{array}$ \\
\hline & & $\begin{array}{l}\text { Postow et al. } \\
\text { (2015) [128] } \\
\text { CheckMate-069 }\end{array}$ & II & $\begin{array}{l}\text { Metastatic melanoma } \\
\text { with no prior } \\
\text { treatment }\end{array}$ & $\begin{array}{l}\text { A. Ipilimumab } 3 \mathrm{mg} / \mathrm{kg}+ \\
\text { nivolumab } 1 \mathrm{mg} / \mathrm{kg} \\
\text { Q3wks followed by } \\
\text { nivolumab } 3 \mathrm{mg} / \mathrm{kg} \\
\text { Q2wks } \\
\text { B. Ipilimumab } 3 \mathrm{mg} / \mathrm{kg}+ \\
\text { placebo Q3wks followed } \\
\text { by placebo Q2wks }\end{array}$ & $\begin{array}{l}142 \\
\text { A. } 95 \\
\text { B. } 47\end{array}$ & - & $\begin{array}{l}\text { A. NR } \\
\text { B. } 4.4 \\
H R=0.4 \\
p<0.001\end{array}$ & $\begin{array}{l}\text { In BRAF } \\
\text { wild-type group: } \\
\text { A. } 61 \\
\text { B. } 11 \\
p<0.001\end{array}$ & $\begin{array}{l}\text { In BRAF } \\
\text { wild-type } \\
\text { group: } \\
\text { A. } 16 \\
\text { B. } 0\end{array}$ & $\begin{array}{l}\text { A. } 54 \% \\
\text { B. } 20 \%\end{array}$ & $\begin{array}{l}\text { Approved in combination } \\
\text { with nivolumab for the } \\
\text { treatment of BRAF V600 } \\
\text { wild type unresectable or } \\
\text { metastatic melanoma } \\
\text { (October 2015) }\end{array}$ \\
\hline & & $\begin{array}{l}\text { Larkin et al. } \\
\text { (2015) [129] } \\
\text { CheckMate } 067\end{array}$ & III & $\begin{array}{l}\text { Previously untreated, } \\
\text { stage III } \\
\text { (unresectable) or } \\
\text { stage IV disease with } \\
\text { known BRAF V600 } \\
\text { mutation status }\end{array}$ & $\begin{array}{c}\text { A: Nivolumab } 1 \mathrm{mg} / \mathrm{kg}+ \\
\text { ipilimumab } 3 \mathrm{mg} / \mathrm{kg} \\
\text { Q3wks., followed by } \\
\text { nivolumab 3mg/kg } \\
\text { Q2wks. } \\
\text { B: Nivolumab 3mg/kg + } \\
\text { placebo Q2wks. } \\
\text { C. Ipilimumab 2mg/kg + } \\
\text { placebo Q3wks. }\end{array}$ & $\begin{array}{c}945 \\
\mathbf{A}=314 \\
\mathbf{B}=316 \\
\mathbf{C}=315\end{array}$ & - & $\begin{array}{c}\text { A. } 11.5(95 \% \text { CI: } \\
8.9-16.7) \\
\text { B. } 6.9(95 \% \mathrm{CI}: \\
4.3-9.5) \\
\text { C. } 2.9(95 \% \mathrm{CI}: \\
2.8-3.4) \\
\text { (HR }=0.42 \\
p<0.001 \text { A vs. C; } \\
\text { HR = } 0.57, \\
p<0.001 \text { B vs. C) }\end{array}$ & $\begin{array}{c}\text { A. } 57.6 \text { (95\% CI: } \\
52.0-63.2) \\
\text { B. } 43.7(95 \% \text { CI: } \\
38.1-49.3) \\
\text { C. } 19.95 \% \text { CI: } \\
14.9-23.8)\end{array}$ & $\begin{array}{l}\text { A. } 11.5 \\
\text { B. } 8.9 \\
\text { C. } 2.2\end{array}$ & $\begin{array}{l}\text { A. } 55.0 \\
\text { B. } 16.3 \\
\text { C. } 27.3\end{array}$ & $\begin{array}{l}\text { Expanded indication: in } \\
\text { combination with } \\
\text { nivolumab for unresectable } \\
\text { or metastatic melanoma } \\
\text { regardless of BRAF V600 } \\
\text { mutation status } \\
\text { (January 2016) }\end{array}$ \\
\hline & & $\begin{array}{l}\text { Eggermont et al. } \\
\text { (2015) [130] } \\
\text { EORTC 18071 }\end{array}$ & III & $\begin{array}{l}\text { Completely resected, } \\
\text { stage III disease }\end{array}$ & $\begin{array}{l}\text { A. Ipilimumab } 10 \mathrm{mg} / \mathrm{kg} \\
\text { Q3wks. for } 4 \text { doses, then } \\
\text { Q3months thereafter } \\
\text { B. Placebo }\end{array}$ & $\begin{array}{c}951 \\
\mathbf{A}=475 \\
\mathbf{B}=476\end{array}$ & - & $\begin{array}{l}\text { A. } 11.6(95 \% \mathrm{CI}: \\
\quad 8.7-15.5) \\
\text { B. } 8.4(95 \% \mathrm{CI}: \\
\quad 7.0-10.8) \\
(\mathrm{HR}=0.75 ; \\
p=0.0013)\end{array}$ & - & - & $\begin{array}{l}\text { A. } 54 \% \\
\text { B. } 25 \%\end{array}$ & $\begin{array}{l}\text { Expanded indication: } \\
\text { adjuvant treatment of } \\
\text { cutaneous melanoma with } \\
\text { pathologic involvement of } \\
\text { regional lymph nodes of } \\
\text { more than } 1 \mathrm{~mm} \text { following } \\
\text { complete resection, } \\
\text { including total } \\
\text { lymphadenectomy } \\
\text { (October 2015) }\end{array}$ \\
\hline
\end{tabular}


Table A2. Cont.

\begin{tabular}{|c|c|c|c|c|c|c|c|c|c|c|c|c|}
\hline Therapy & Cancer & Study & Phase & Stage of Disease & Treatment Regimen & $\mathbf{N}$ & $\begin{array}{c}\text { Median OS } \\
\text { (Months) }\end{array}$ & $\begin{array}{l}\text { Median PFS } \\
\text { (Months) }\end{array}$ & ORR (\%) & CRR (\%) & $\begin{array}{c}\text { Grade } \\
\text { III/IV } \\
\text { AEs (\%) }\end{array}$ & $\begin{array}{l}\text { Resultant FDA-Approved } \\
\text { Indication }\end{array}$ \\
\hline & & $\begin{array}{l}\text { Merchant et al. } \\
\text { (2016) [131] }\end{array}$ & I & $\begin{array}{l}\leq 21 \text { years of age with } \\
\text { progressive or } \\
\text { recurrent solid } \\
\text { tumors }\end{array}$ & $\begin{array}{c}\text { Dose escalation cohorts } \\
\text { given ipilimumab } 1,3,5, \\
\text { or } 10 \mathrm{mg} / \mathrm{m}^{2} \mathrm{IV} \mathrm{Q} 3 \mathrm{wks} \text { in } \\
3+3 \text { design }\end{array}$ & 33 & - & - & 0 & 0 & $27 \%$ & \\
\hline & & $\begin{array}{l}\text { Geoerger et at. } \\
\text { (2017) [132] }\end{array}$ & II & $\begin{array}{l}\text { Patients ages 12-18 } \\
\text { with unresectable } \\
\text { stage III or IV } \\
\text { malignant melanoma }\end{array}$ & $\begin{array}{c}\text { A. Ipilimumab } 3 \mathrm{mg} / \mathrm{kg} \\
\text { Q3wks } \\
\text { B. Ipilimumab } 10 \mathrm{mg} / \mathrm{kg} \\
\text { Q3wks }\end{array}$ & $\begin{array}{c}12 \\
A=4 \\
B=8\end{array}$ & $\begin{array}{l}\text { A. } 18.2(95 \% \mathrm{CI}: \\
\quad 8.9-18.2) \\
\text { B. NR (95\% CI: } \\
\quad \text { 5.2-NR) }\end{array}$ & $\begin{array}{l}\text { A. } 2.6 \text { months } \\
(95 \% \text { CI: } 2.3-8.5) \\
\text { B. } 2.9 \text { months } \\
(95 \% \text { CI: } 0.7-\mathrm{NR})\end{array}$ & $\begin{array}{l}\text { Overall: } 17 \\
\text { A: } 0 \\
\text { B: } 28.6\end{array}$ & $\begin{array}{l}\text { A: } 0 \\
\text { B: } 0\end{array}$ & $\begin{array}{l}\text { A. } 25 \\
\text { B. } 62.5\end{array}$ & $\begin{array}{c}\text { Approval expanded to } \\
\text { include pediatric patients } 12 \\
\text { years and older with } \\
\text { unresectable or metastatic } \\
\text { melanoma } \\
\end{array}$ \\
\hline & & $\begin{array}{l}\text { Long et al. } \\
\text { (2018) [133] }\end{array}$ & II & $\begin{array}{l}\text { Stage IV disease with } \\
\text { active brain } \\
\text { metastases } \\
\text { (Groups } \\
\text { A,B-asymptomatic } \\
\text { and/or CNS } \\
\text { treatment naïve; } \\
\text { Group } \\
\text { C-symptomatic } \\
\text { and/or failed prior } \\
\text { treatment) }\end{array}$ & $\begin{array}{c}\text { A. Nivolumab 1mg/kg + } \\
\text { ipilimumab 3mg/kg } \\
\text { Q3wks x4, followed by } \\
\text { nivolumab 3mg/kg } \\
\text { Q2wks. } \\
\text { B. Nivolumab 3mg } / \mathrm{kg} \\
\text { Q2wks. } \\
\text { C. Nivolumab 3mg } / \mathrm{kg} \\
\text { Q2wks. }\end{array}$ & $\begin{array}{c}79 \\
\mathbf{A}=36 \\
\mathbf{B}=27 \\
\mathbf{C}=16\end{array}$ & - & - & $\begin{array}{c}\text { A. } 46 \text { (95\% CI: } \\
29-63) \\
\text { B. } 20(95 \% \text { CI: } \\
7-41) \\
\text { C: } 6(95 \% \text { CI: } \\
0-30)\end{array}$ & $\begin{array}{l}\text { A. } 19 \\
\text { B. } 12 \\
\text { C. } 0\end{array}$ & $\begin{array}{l}\text { A. } 62 \\
\text { B. } 44 \\
\text { C. } 9\end{array}$ & 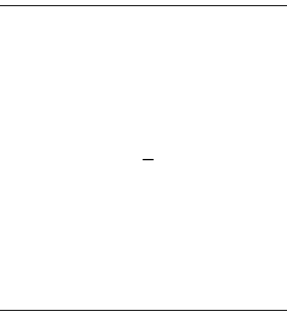 \\
\hline & \multirow[t]{2}{*}{ NSCLC } & $\begin{array}{l}\text { Lynch et al. } \\
\text { (2012) [41] }\end{array}$ & II & $\begin{array}{c}\text { Chemotherapy naïve } \\
\text { disease }\end{array}$ & $\begin{array}{c}\text { A. Paclitaxel + } \\
\text { carboplatin + placebo } \\
\text { B. Paclitaxel + } \\
\text { carboplatin + concurrent } \\
\text { ipilimumab 10mg/kg } \\
\text { Q3wks. x4, then } \\
\text { paclitaxel + carboplatin + } \\
\text { placebo Q3wks. } 2 \text { 2 } \\
\text { C. Paclitaxel + } \\
\text { carboplatin + placebo } \\
\text { Q3wks. } 2 \text { 2, then addition } \\
\text { of ipilimumab to } \\
\text { regimen at } 10 \mathrm{mg} / \mathrm{kg} \\
\text { Q3wks. x4 }\end{array}$ & $\begin{array}{c}204 \\
\mathbf{A}=66 \\
\mathbf{B}=70 \\
\mathbf{C}=68\end{array}$ & 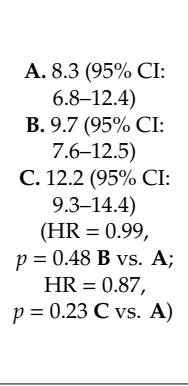 & $\begin{array}{c}\text { A. } 4.2(95 \% \text { CI: } \\
2.8-5.3) \\
\text { B. } 4.1(95 \% \text { CI: } \\
2.8-5.3) \\
\text { C. } 5.1(95 \% \text { CI: } \\
4.2-5.7) \\
(\text { HR }=0.88 \\
p=0.25 \text { B vs. } \mathbf{A} ; \\
\text { HR = } 0.69 ; \\
p=0.02 \text { C vs. A) }\end{array}$ & $\begin{array}{c}\text { A; } 14 \text { (95\% CI: } \\
6-24) \\
\text { B: } 21(95 \% \text { CI: } \\
13-33) \\
\text { C: } 32 \text { (95\% CI: } \\
22-45)\end{array}$ & $\begin{array}{l}\text { A. } 0 \\
\text { B. } 0 \\
\text { C. } 0\end{array}$ & $\begin{array}{l}\text { A. } 37 \\
\text { B. } 41 \\
\text { C. } 39\end{array}$ & - \\
\hline & & $\begin{array}{l}\text { Govindan et al. } \\
\quad(2017)[42]\end{array}$ & III & $\begin{array}{l}\text { Metastatic or } \\
\text { recurrent disease, } \\
\text { chemotherapy naïve }\end{array}$ & $\begin{array}{c}\text { A. Paclitaxel + } \\
\text { carboplatin + } \\
\text { ipilimumab 10mg/kg } \\
\text { Q3wks., then ipilimumab } \\
\text { maintenance Q12wks. } \\
\text { B. Paclitaxel + } \\
\text { carboplatin + placebo } \\
\text { Q3wks., then placebo } \\
\text { maintenance Q12wks. }\end{array}$ & $\begin{array}{c}749 \\
\mathbf{A}=388 \\
\mathbf{B}=361\end{array}$ & $\begin{array}{c}\text { A. } 13.4 \\
\text { B. } 12.4 \\
(\mathrm{HR}=0.9 \\
p=0.25)\end{array}$ & $\begin{array}{c}\text { A. } 5.6 \\
\text { B. } 5.6 \\
(\mathrm{HR}=0.87)\end{array}$ & $\begin{array}{l}\text { A. } 45.3(95 \% \text { CI: } \\
\text { 36.9-54.0) } \\
\text { B. } 26.9(95 \% \text { CI: } \\
20.2-34.4)\end{array}$ & $\begin{array}{l}\text { A. }<1 \\
\text { B. }<1\end{array}$ & $\begin{array}{l}\text { A. } 51 \\
\text { B. } 35\end{array}$ & - \\
\hline
\end{tabular}


Table A2. Cont.

\begin{tabular}{|c|c|c|c|c|c|c|c|c|c|c|c|c|}
\hline Therapy & Cancer & Study & Phase & Stage of Disease & Treatment Regimen & $\mathbf{N}$ & $\begin{array}{c}\text { Median OS } \\
\text { (Months) }\end{array}$ & $\begin{array}{c}\text { Median PFS } \\
\text { (Months) }\end{array}$ & ORR (\%) & CRR (\%) & $\begin{array}{c}\text { Grade } \\
\text { III/IV } \\
\text { AEs (\%) }\end{array}$ & $\begin{array}{l}\text { Resultant FDA-Approved } \\
\text { Indication }\end{array}$ \\
\hline & & $\begin{array}{l}\text { Hellman et al. } \\
\text { (2018) [134] } \\
\text { CheckMate 227 }\end{array}$ & III & $\begin{array}{l}\text { Metastatic or } \\
\text { recurrent disease, } \\
\text { high mutational } \\
\text { burden }\end{array}$ & $\begin{array}{c}\text { A. Nivolumab 3mg/kg } \\
\text { Q2wks. + ipilimumab } \\
\text { 1mg/kg Q6wks. } \\
\text { B. Platinum doublet } \\
\text { therapy Q3wks. } \\
\text { C. Nivolumab 240mg } \\
\text { Q2wks. }\end{array}$ & $\begin{array}{l}1739 \\
(1: 1: 1 \\
\text { ratio) }\end{array}$ & - & $\begin{array}{c}\text { A: } 7.2(95 \% \text { CI: } \\
5.5-13.2) \\
\text { B: } 5.5(95 \% \text { CI: } \\
4.4-5.8) \\
\text { C: } 4.2(95 \% \text { CI: } \\
2.7-8.3) \\
\text { (HR = 0.58, } \\
p<0.001 \text { A vs. B; } \\
\text { HR }=0.95 \\
p=0.78 \text { C vs. B) }\end{array}$ & $\begin{array}{l}\text { A. } 45.3(95 \% \mathrm{CI}: \\
36.9-54.0) \\
\text { B. } 26.9(95 \% \mathrm{CI}: \\
20.2-34.4)\end{array}$ & $\begin{array}{l}\text { A: } 3.6 \\
\text { B: } 0.6\end{array}$ & $\begin{array}{l}\text { A: } 31.2 \\
\text { B: } 36.1 \\
\text { C: } 18.9\end{array}$ & - \\
\hline & $\begin{array}{l}\text { Prostate } \\
\text { cancer }\end{array}$ & $\begin{array}{l}\text { Slovin et al. } \\
\text { (2013) [135] }\end{array}$ & I/II & $\begin{array}{l}\text { Metastatic castration } \\
\text { resistant disease }\end{array}$ & $\begin{array}{l}\text { Ipilimumab } 2 \mathrm{mg} / \mathrm{kg} \text {, } \\
5 \mathrm{mg} / \mathrm{kg}, \text { or } 10 \mathrm{mg} / \mathrm{kg} \\
\text { Q3wks. +/- radiation }\end{array}$ & 71 & $\begin{array}{l}17.4(95 \% \text { CI: } \\
11.5-24.7)\end{array}$ & - & - & 4 & 32 & - \\
\hline & RCC & $\begin{array}{c}\text { Motzer et al. } \\
\text { (2018) [39] } \\
\text { CheckMate 214 }\end{array}$ & III & $\begin{array}{l}\text { Advanced, untreated } \\
\text { disease }\end{array}$ & $\begin{array}{l}\text { A. Nivolumab 3mg } / \mathrm{kg}+ \\
\text { ipilimumab } 1 \mathrm{mg} / \mathrm{kg} \\
\text { Q2wks. } \\
\text { B. Sunitinib 50mg QD for } \\
4 \text { wks. }\end{array}$ & $\begin{array}{c}1096 \\
\mathbf{A}=550 \\
\mathbf{B}=546\end{array}$ & $\begin{array}{c}\text { A. NR }(95 \% \text { CI: } \\
28.2-\mathrm{NR}) \\
\text { B. } 26.0 \text { (95\% CI: } \\
22.1-\mathrm{NR}) \\
(\mathrm{HR}=0.63 \\
p<0.001) \\
\end{array}$ & $\begin{array}{c}\text { A. } 11.6(95 \% \mathrm{CI} \text { : } \\
8.7-15.5) \\
\text { B. } 8.4(95 \% \mathrm{CI}: \\
7.0-10.8) \\
(\mathrm{HR}=0.82 \\
p=0.03) \\
\end{array}$ & $\begin{array}{c}\text { A. } 42(95 \% \mathrm{CI}: \\
\quad 37-47) \\
\text { B. } 27(95 \% \mathrm{CI}: \\
22-31) \\
(p<0.001)\end{array}$ & $\begin{array}{l}\text { A. } 9 \\
\text { B. } 1\end{array}$ & $\begin{array}{l}\text { A: } 46 \\
\text { B: } 63\end{array}$ & $\begin{array}{l}\text { Combination } \\
\text { ipilimumab/nivolumab for } \\
\text { intermediate- or poor-risk, } \\
\text { previously untreated } \\
\text { advanced RCC (April 2018) }\end{array}$ \\
\hline & SCLC & $\begin{array}{l}\text { Reck et al. } \\
\text { (2013) [136] }\end{array}$ & II & $\begin{array}{l}\text { Chemotherapy naïve, } \\
\text { extensive disease }\end{array}$ & $\begin{array}{c}\text { A. Paclitaxel + } \\
\text { carboplatin + placebo } \\
\text { B. Paclitaxel + } \\
\text { carboplatin + concurrent } \\
\text { ipilimumab 10m//kg } \\
\text { Q3wks. x4, then } \\
\text { paclitaxel + carboplatin + } \\
\text { placebo Q3wks. } x 2 \\
\text { C. Paclitaxel }+ \\
\text { carboplatin Q3wks. x2, } \\
\text { then addition of } \\
\text { ipilimumab to regimen } \\
\text { at 10mg/kg Q3wks. x4 } \\
\end{array}$ & $\begin{array}{c}130 \\
\mathbf{A}=45 \\
\mathbf{B}=43 \\
\mathbf{C}=42\end{array}$ & $\begin{array}{c}\text { A. } 9.9 \text { (95\% CI: } \\
\text { 8.6-11.7) } \\
\text { B: } 9.1(95 \% \text { CI: } \\
\text { 6.7-13.0) } \\
\text { C: } 12.9 .9(95 \% \text { CI: } \\
7.9-16.5) \\
\text { (HR = } 0.95, \\
p=0.41 \text { B vs. A; } \\
\text { HR = 0.75, } \\
p=0.13 \text { C vs. A) }\end{array}$ & $\begin{array}{c}\text { A. } 5.3 \text { (95\% CI: } \\
4.7-5.7) \\
\text { B. } 5.7(95 \% \text { CI: } \\
5.2-6.9) \\
\text { C. } 6.4(95 \% \text { CI: } \\
5.3-7.8) \\
(\mathrm{HR}=0.75, \\
p=0.11 \text { B vs. A; } \\
\text { HR = 0.64, } \\
p=0.03 \text { C vs. A) }\end{array}$ & $\begin{array}{c}\text { A. } 49(95 \% \text { CI: } \\
34-64) \\
\text { B. } 33(95 \% \text { CI: } \\
19-49) \\
\text { C. } 57(95 \% \text { CI: } \\
41-72)\end{array}$ & $\begin{array}{l}\text { A: } 0 \\
\text { B: } 2 \\
\text { C: } 0\end{array}$ & $\begin{array}{l}\text { A: } 30 \\
\text { B: } 43 \\
\text { C. } 50\end{array}$ & - \\
\hline & & $\begin{array}{l}\text { Reck et al. } \\
\text { (2016) [137] } \\
\text { CA184-156 }\end{array}$ & III & $\begin{array}{l}\text { Newly diagnosed, } \\
\text { extensive disease }\end{array}$ & $\begin{array}{l}\text { A. Chemotherapy with } \\
\text { etoposide and either } \\
\text { cisplatin or carboplatin } \\
\text { plus ipilimumama } \\
\text { 10mg/kg Q3wks. } \\
\text { followed by ipilimumab } \\
\text { maintenance Q12wks. } \\
\text { B. Chemotherapy with } \\
\text { etoposide and either } \\
\text { cisplatin or carboplatin } \\
\text { plus placebo Q3wks. } \\
\text { followed by placebo } \\
\text { maintenance Q12wks. }\end{array}$ & $\begin{array}{c}954 \\
\mathbf{A}=478 \\
\mathbf{B}=476\end{array}$ & $\begin{array}{c}\text { A. } 11.0(95 \% \text { CI: } \\
10.5-11.3) \\
\text { B. } 10.9(95 \% \text { CI: } \\
10.0-11.5) \\
(\mathrm{HR}=0.94 \\
p=0.38)\end{array}$ & $\begin{array}{c}\text { A. } 4.6 \text { ( } 95 \% \text { CI: } \\
4.5-5.0) \\
\text { B. } 4.4(95 \% \text { CI: } \\
4.4-4.6) \\
(\mathrm{HR}=0.85 ; \\
p=0.02)\end{array}$ & $\begin{array}{l}\text { A. } 62(95 \% \text { CI: } \\
\quad 58-67) \\
\text { B: } 62(95 \% \text { CI: } \\
58-67)\end{array}$ & $\begin{array}{l}\text { A: }<1 \\
\text { B: } 0\end{array}$ & $\begin{array}{l}\text { A: } 48 \\
\text { B: } 44\end{array}$ & - \\
\hline
\end{tabular}


Table A2. Cont.

\begin{tabular}{|c|c|c|c|c|c|c|c|c|c|c|c|c|}
\hline Therapy & Cancer & Study & Phase & Stage of Disease & Treatment Regimen & $\mathbf{N}$ & $\begin{array}{l}\text { Median OS } \\
\text { (Months) }\end{array}$ & $\begin{array}{l}\text { Median PFS } \\
\text { (Months) }\end{array}$ & ORR (\%) & CRR (\%) & $\begin{array}{c}\text { Grade } \\
\text { III/IV } \\
\text { AEs (\%) }\end{array}$ & $\begin{array}{l}\text { Resultant FDA-Approved } \\
\text { Indication }\end{array}$ \\
\hline \multirow[t]{4}{*}{$\underline{\text { Tremelimumab }}$} & Melanoma & $\begin{array}{l}\text { Ribas et al. } \\
\text { (2013) [138] }\end{array}$ & III & $\begin{array}{l}\text { Treatment naive } \\
\text { unresectable stage } \\
\text { IIIc/IV disease }\end{array}$ & $\begin{array}{l}\text { A. Tremelimumab } \\
15 \mathrm{mg} / \mathrm{kg} \text { Q90days } \\
\text { B. Physician's choice of } \\
\text { standard of care } \\
\text { chemotherapy }\end{array}$ & $\begin{array}{c}655 \mathbf{A}=328 \\
\mathbf{B}=327\end{array}$ & $\begin{array}{c}\text { A. } 12.58 \\
\text { B. } 10.71 \\
\text { HR }=0.88 \\
p=0.127\end{array}$ & - & $\begin{array}{c}\text { A. } 10.7(95 \% \text { CI: } \\
\quad 7.8-14.9) \\
\text { B. } 9.8(-15 \% \text { CI: } \\
\quad 6.8-13.5)\end{array}$ & $\begin{array}{l}\text { A. } 3 \\
\text { B. } 2\end{array}$ & $\begin{array}{l}\text { A. } 52 \\
\text { B. } 37\end{array}$ & \\
\hline & Mesothelioma & $\begin{array}{l}\text { Calabro et al. } \\
\text { (2015) [139] }\end{array}$ & II & $\begin{array}{l}\text { Chemotherapy-resistant, } \\
\text { stage III disease }\end{array}$ & $\begin{array}{l}\text { Tremelimumab } 10 \mathrm{mg} / \mathrm{kg} \\
\text { Q4wks. x6, then Q12wks. }\end{array}$ & 29 & $\begin{array}{l}11.3(95 \% \mathrm{CI}: \\
3.4-19.2)\end{array}$ & $\begin{array}{l}6.2(95 \% \text { CI: } \\
5.7-6.7)\end{array}$ & - & 0 & 7 & $\begin{array}{l}\text { Orphan Drug Designation } \\
\text { for treatment of malignant } \\
\text { mesothelioma (April 2015) }\end{array}$ \\
\hline & & $\begin{array}{l}\text { Maio et al. } \\
\text { (2017) [43] }\end{array}$ & $\mathrm{IIb}$ & $\begin{array}{l}\text { Unresectable pleural } \\
\text { or peritoneal disease }\end{array}$ & $\begin{array}{c}\text { A. Tremelimumab } \\
\text { 10mg/kg Q4wks. x4, then } \\
\text { Q12wks. } \\
\text { B. Placebo }\end{array}$ & $\begin{array}{c}568 \mathbf{A}=382 \\
\mathbf{B}=189\end{array}$ & $\begin{array}{c}\text { A. } 7.7(95 \% \text { CI: } \\
\quad 6.8-8.9) \\
\text { B. } 7.3(95 \% \text { CI: } \\
\quad 6.8-8.9) \\
(\mathrm{HR}=0.92 \\
p=0.41)\end{array}$ & $\begin{array}{c}\text { A. } 2.8(95 \% \text { CI: } \\
2.8-2.8) \\
\text { B. } 2.7(95 \% \text { CI: } \\
2.7-2.8) \\
(\mathrm{HR}=0.81 \\
p=0.03)\end{array}$ & $\begin{array}{l}\text { A. } 4.5(95 \% \mathrm{CI}: \\
2.6-7.0) \\
\text { B. } 1.1(95 \% \mathrm{CI}: \\
0.1-3.8)\end{array}$ & $\begin{array}{l}\text { A. } 0 \\
\text { B. } 0\end{array}$ & $\begin{array}{l}\text { A. } 65 \\
\text { B. } 48\end{array}$ & - \\
\hline & NSCLC & $\begin{array}{l}\text { Rizvi et al. } \\
\text { (2018) [140] }\end{array}$ & III & $\begin{array}{c}\text { Chemotherapy naïve } \\
\text { disease }\end{array}$ & $\begin{array}{l}\text { A. Durvalumab 20mg/kg } \\
\text { Q4wks } \\
\text { B. Durvalumab 20mg/kg } \\
\text { + tremelimumab } 1 \mathrm{mg} / \mathrm{kg} \\
\text { Q4wks. } \\
\begin{array}{c}\text { C. Chemotherapy x6 } \\
\text { cycles }\end{array}\end{array}$ & $\begin{array}{l}488(1: 1: 1 \\
\text { ratio })\end{array}$ & $\begin{array}{c}\text { A. } 16.3(97.5 \% \text { CI: } \\
0.56-1.02) \\
\text { B. } 11.9(98.8 \% \text { CI: } \\
0.61-1.17) \\
\text { C. } 12.9 \\
(\mathrm{HR}=0.76 \\
p=0.036 \text { A vs. C; } \\
\text { HR }=0.85 \\
p=0.202 \text { B vs. C) }\end{array}$ & $\begin{array}{c}\text { A. }- \\
\text { B. } 3.9(99.5 \% \text { CI: } \\
0.72-1.53) \\
\text { C. } 5.4 \\
\text { (HR = } 1.05 ; \\
p=0.705 \text { B vs. C) }\end{array}$ & - & - & $\begin{array}{l}\text { A: } 14.6 \\
\text { B: } 22.1 \\
\text { C: } 33.8\end{array}$ & - \\
\hline
\end{tabular}

$\mathrm{CI}=$ confidence interval, $\mathrm{CRC}=$ colorectal cancer, $\mathrm{HR}=$ hazard ratio, $\mathrm{NR}=$ not reached, $\mathrm{NSCLC}=$ non-small cell lung cancer, $\mathrm{RCC}=$ renal cell carcinoma, $\mathrm{SCLC}=$ small cell lung cancer. 
Table A3. Select clinical data for nivolumab.

\begin{tabular}{|c|c|c|c|c|c|c|c|c|c|c|c|}
\hline Cancer & Study & Phase & Stage of Disease & Treatment Regimen & $\mathbf{N}$ & $\begin{array}{l}\text { Median OS } \\
\text { (Months) }\end{array}$ & $\begin{array}{l}\text { Median PFS } \\
\text { (Months) }\end{array}$ & ORR (\%) & CRR (\%) & $\begin{array}{l}\text { Grade } \\
\text { III/IV AEs } \\
(\%)\end{array}$ & $\begin{array}{l}\text { Resultant FDA-Approved } \\
\text { Indication }\end{array}$ \\
\hline Anal SCC & $\begin{array}{l}\text { Morris et al. } \\
\text { (2017) [141] }\end{array}$ & II & $\begin{array}{c}\text { Treatment refractory } \\
\text { metastatic squamous } \\
\text { cell cancer of the anal } \\
\text { canal }\end{array}$ & $\begin{array}{c}\text { Nivolumab } 3 \mathrm{mg} / \mathrm{kg} \\
\text { Q2wks }\end{array}$ & 37 & $\begin{array}{l}11.5 \text { (95\% CI: } \\
\text { 7.1-NE) }\end{array}$ & $\begin{array}{l}4.1 \text { ( } 95 \% \text { CI: } \\
\text { 3.0-7.9) }\end{array}$ & $\begin{array}{l}24 \text { (95\% CI: } \\
15-33)\end{array}$ & 5.4 & 13.5 & - \\
\hline Bladder cancer & $\begin{array}{l}\text { Sharma et al. } \\
\text { (2017) [44] } \\
\text { CheckMate } \\
275\end{array}$ & II & $\begin{array}{l}\text { Metastatic urothelial } \\
\text { carcinoma, history of } \geq 1 \\
\text { treatment of platinum } \\
\text { therapy }\end{array}$ & $\begin{array}{c}\text { Nivolumab 3mg/kg } \\
\text { Q2wks. }\end{array}$ & 270 & $\begin{array}{l}\text { All. } 8.7 \text { (95\% CI: } \\
\text { 6.1-NE) } \\
\text { PD-L1+. 11.30 } \\
\text { (95\% CI 8.7-NE) } \\
\text { PD-L1- . . } \\
\text { (4.3-8.1) }\end{array}$ & $\begin{array}{l}\text { All. } 2 \text { months }(95 \% \\
\text { CI: } 1.9-2.6)\end{array}$ & $\begin{array}{c}\text { All. } 19.6 \\
\text { (95\% CI: } \\
15.0-24.9)\end{array}$ & All. $2 \%$ & All. $18.0 \%$ & $\begin{array}{l}\text { Locally advanced or } \\
\text { metastatic urothelial } \\
\text { carcinoma with disease } \\
\text { progression during or } \\
\text { following platinum } \\
\text { chemotherapy or disease } \\
\text { progression within } 12 \text { months } \\
\text { of neoadjuvant or adjuvant } \\
\text { platinum chemotherapy } \\
\text { (February 2017) }\end{array}$ \\
\hline \multirow[t]{2}{*}{ CRC } & $\begin{array}{l}\text { Overman et al. } \\
\text { (2017) [45] }\end{array}$ & II & $\begin{array}{l}\text { Recurrent or metastatic } \\
\text { dMMR/MSI-H disease }\end{array}$ & $\begin{array}{c}\text { Nivolumab 3mg/kg } \\
\text { Q2wks. }\end{array}$ & 74 & NR & $\begin{array}{c}14.3(95 \% \mathrm{CI}: \\
4.3-\mathrm{NE})\end{array}$ & $\begin{array}{l}31 \text { (95\% CI: } \\
20.8-42.9)\end{array}$ & 0 & 21.0 & $\begin{array}{l}\text { Patients } 12 \text { years and older } \\
\text { with dMMR/MSI-H metastatic } \\
\text { CRC that has progressed } \\
\text { following treatment with a } \\
\text { fluoropyrimidine, oxaliplatin, } \\
\text { and irinotecan (August 2017) }\end{array}$ \\
\hline & $\begin{array}{l}\text { Overmanet al. } \\
\text { (2018) [127] } \\
\text { CheckMate } \\
\quad 142\end{array}$ & II & $\begin{array}{l}\text { Recurrent of metastatic } \\
\text { dMMR/MSI-H disease }\end{array}$ & $\begin{array}{c}\text { Nivolumab } 3 \mathrm{mg} / \mathrm{kg}+ \\
\text { ipilimumab } 1 \mathrm{mg} / \mathrm{kg} \\
\text { Q3wks } 4 \text { followed by } \\
\text { nivolumab } 3 \mathrm{mg} / \mathrm{kg} \\
\text { Q2wks } \\
\end{array}$ & 119 & NR & NR & $\begin{array}{l}49 \text { (95\% CI: } \\
39.5-58.1)\end{array}$ & 4 & 32.0 & $\begin{array}{l}\text { Approved in combination } \\
\text { with ipilimumab for } \\
\text { previously treated } \\
\text { MSI-H/dMMR metastatic } \\
\text { CRC (July 2018) }\end{array}$ \\
\hline $\begin{array}{l}\text { Gastric or GEJ } \\
\text { cancer }\end{array}$ & $\begin{array}{l}\text { Kang et al. } \\
\text { (2017) [142] }\end{array}$ & III & $\begin{array}{l}\text { Unresectable advanced } \\
\text { or recurrent disease } \\
\text { refractory to, or } \\
\text { intolerant of, standard } \\
\text { therapy }\end{array}$ & $\begin{array}{c}\text { A. Nivolumab } 3 \mathrm{mg} / \mathrm{kg} \\
\text { Q2wks } \\
\text { B. Placebo }\end{array}$ & $\begin{array}{c}493 \\
\mathbf{A}=330 \\
\mathbf{B}=163\end{array}$ & $\begin{array}{l}\text { A. } 5.26(95 \% \text { CI: } \\
4.60-6.37) \\
\text { B. } 4.14(95 \% \text { CI: } \\
3.42-4.86) \\
\text { HR }=0.63 \\
p<0.0001 \\
\end{array}$ & $\begin{array}{l}\text { A. } 1.61(95 \% \text { CI: } \\
1.54-2.30) \\
\text { B. } 1.45(95 \% \text { CI: } \\
1.45-1.54) \\
\text { HR }=0.60 \\
p<0.0001\end{array}$ & $\begin{array}{l}\text { A. } 11.2 \\
(95 \% \text { CI: } \\
7.7-15.6) \\
\text { B. } 0(95 \% \\
\text { CI: } 0-2.8)\end{array}$ & $\begin{array}{l}\text { A. } 0 \\
\text { B. } 0\end{array}$ & $\begin{array}{l}\text { A. } 10 \\
\text { B. } 4\end{array}$ & - \\
\hline \multirow[t]{3}{*}{ GBM } & $\begin{array}{l}\text { Reardon etal. } \\
\text { (2017) [143] } \\
\text { Checkmate } \\
\quad 143\end{array}$ & III & $\begin{array}{l}\text { Patients with first } \\
\text { recurrence of GBM }\end{array}$ & $\begin{array}{c}\text { A. Nivolumab } 3 \mathrm{mg} / \mathrm{kg} \\
\text { Q2wks } \\
\text { B. Bevacizumab } 10 \mathrm{mg} / \mathrm{kg} \\
\text { Q2wks }\end{array}$ & $\begin{array}{c}369 \\
\mathbf{A}=184 \\
\mathbf{B}=185\end{array}$ & $\begin{array}{c}\text { A. } 9.8(95 \% \mathrm{CI}: \\
\quad 8.2-11.8) \\
\text { B. } 10.0(95 \% \mathrm{CI}: \\
\quad 9.0-11.8) \\
\mathrm{HR}=1.04, p=0.76\end{array}$ & $\begin{array}{l}\text { A. } 1.5(95 \% \text { CI: } \\
1.5-1.5) \\
\text { B. } 3.5(95 \% \text { CI: } \\
2.9-4.6) \\
\mathrm{HR}=1.97 \\
p<0.0001\end{array}$ & $\begin{array}{l}\text { A. } 8 \\
\text { B. } 23\end{array}$ & - & $\begin{array}{l}\text { A. } 18 \\
\text { B. } 15\end{array}$ & - \\
\hline & $\begin{array}{l}\text { CheckMate } \\
498 \\
\text { (2019) [144] }\end{array}$ & III & $\begin{array}{l}\text { Centrally confirmed } \\
\text { treatment naive } \\
\text { MGMT-unmethylated } \\
\text { disease }\end{array}$ & $\begin{array}{l}\text { A. Nivolumab + radiation } \\
\text { B. Temozolomide + } \\
\text { radiation }\end{array}$ & $\sim 550$ & $\begin{array}{l}\text { Primary endpoint } \\
\text { of OS not met }\end{array}$ & - & - & - & - & - \\
\hline & $\begin{array}{c}\text { Checkmate } \\
548 \\
\text { (2019) [145] }\end{array}$ & III & $\begin{array}{l}\text { Newly diagnosed } \\
\text { MGMT-methylated } \\
\text { disease }\end{array}$ & $\begin{array}{l}\text { A. Nivolumab + radiation } \\
+ \text { temozolomide } \\
\text { B. Radiation }+ \\
\text { temozolomide (standard } \\
\text { of care) }\end{array}$ & & - & $\begin{array}{l}\text { Primary endpoint } \\
\text { of PFS not met }\end{array}$ & - & - & - & - \\
\hline
\end{tabular}


Table A3. Cont.

\begin{tabular}{|c|c|c|c|c|c|c|c|c|c|c|c|}
\hline Cancer & Study & Phase & Stage of Disease & Treatment Regimen & $\mathbf{N}$ & $\begin{array}{l}\text { Median OS } \\
\text { (Months) }\end{array}$ & $\begin{array}{l}\text { Median PFS } \\
\text { (Months) }\end{array}$ & ORR (\%) & CRR (\%) & $\begin{array}{l}\text { Grade } \\
\text { III/IV AEs } \\
(\%)\end{array}$ & $\begin{array}{l}\text { Resultant FDA-Approved } \\
\text { Indication }\end{array}$ \\
\hline HCC & $\begin{array}{l}\text { El-Khoueiry et al. } \\
\text { (2017) [47] } \\
\text { CheckMate } \\
040\end{array}$ & $\mathrm{I} / \mathrm{II}$ & Advanced disease & $\begin{array}{c}\text { Nivolumab 3mg/kg } \\
\text { Q2wks. }\end{array}$ & 214 & - & $\begin{array}{l}4.0 \text { (95\% CI: } \\
2.9-5.4)\end{array}$ & $\begin{array}{l}20 \text { (95\% CI: } \\
\quad 15-26)\end{array}$ & 1.0 & 19.0 & $\begin{array}{l}\text { Advanced HCC in patients } \\
\text { who have been previously } \\
\text { treated with sorafenib } \\
\text { (September 2017) }\end{array}$ \\
\hline \multirow[t]{2}{*}{ HL } & $\begin{array}{l}\text { Ansell et al. } \\
(2015)[49] \\
\text { CheckMate } \\
039\end{array}$ & I & $\begin{array}{l}\text { Relapsed or refractory } \\
\text { disease }\end{array}$ & $\begin{array}{l}\text { Nivolumab 3mg/kg on } \\
\text { week } 1 \text {, week 4, then } \\
\text { Q2wks. thereafter }\end{array}$ & 23 & - & - & $\begin{array}{c}87(95 \% \text { CI: } \\
\quad 66-97)\end{array}$ & 17 & 52.0 & - \\
\hline & $\begin{array}{l}\text { Younes et al. } \\
\text { (2016) [50] } \\
\text { CheckMate } \\
205\end{array}$ & II & $\begin{array}{l}\text { Relapsed refractory } \\
\text { classical HL following } \\
\text { ASCT and BV }\end{array}$ & $\begin{array}{c}\text { Nivolumab 3mg/kg } \\
\text { Q2wks. }\end{array}$ & 80 & - & $\begin{array}{l}10.0 \text { (95\% CI: } \\
\text { 8.4-NE) }\end{array}$ & $\begin{array}{l}66.3(95 \% \\
\text { CI: } \\
54.8-76.4)\end{array}$ & 9.0 & 25.0 & $\begin{array}{l}\text { cHL that has relapsed or } \\
\text { progressed after ASCT and } \\
\text { post-transplant BV (May } \\
\text { 2016) }\end{array}$ \\
\hline HNSCC & $\begin{array}{l}\text { Ferris et al. } \\
\text { (2016) [46] } \\
\text { CheckMate } \\
141\end{array}$ & III & $\begin{array}{l}\text { Recurrent or metastatic } \\
\text { disease }\end{array}$ & $\begin{array}{c}\text { A: Nivolumab } 3 \mathrm{mg} / \mathrm{kg} \\
\text { Q2wks. } \\
\text { B: Standard single agent } \\
\text { chemotherapy (cetuximab, } \\
\text { MTX, docetaxel) }\end{array}$ & $\begin{array}{c}361 \\
\mathbf{A}=240 \\
\mathbf{B}=121\end{array}$ & $\begin{array}{c}\text { A. } 7.5 \text { (95\% CI: } \\
5.5-9.1) \\
\text { B. } 5.1(95 \% \text { CI: } \\
4.0-6.0)(\mathrm{HR}=0.70 \\
p=0.01)\end{array}$ & $\begin{array}{c}\text { A. } 2.0(95 \% \text { CI: } \\
1.9-2.1) \\
\text { B: } 2.3(95 \% \text { CI: } \\
1.9-3.1) \\
(\mathrm{HR}=0.89 \\
p=0.32) \\
\end{array}$ & $\begin{array}{l}\text { A. } 13.3 \\
(95 \% \text { CI: } \\
9.3-18.3) \\
\text { B. } 5.8 \\
(2.4-11.6)\end{array}$ & $\begin{array}{l}\text { A. } 2.5 \\
\text { B. } 0.8\end{array}$ & $\begin{array}{l}\text { A. } 13.1 \\
\text { B. } 35.1\end{array}$ & $\begin{array}{l}\text { Recurrent or metastatic } \\
\text { HNSCC with disease } \\
\text { progression on or after a } \\
\text { platinum-based therapy } \\
\text { (November 2016) }\end{array}$ \\
\hline \multirow[t]{3}{*}{ Melanoma } & $\begin{array}{l}\text { Robert et al. } \\
\text { (2014) [65] } \\
\text { CheckMate } \\
066\end{array}$ & III & $\begin{array}{l}\text { Metastatic disease w/o } \\
\text { BRAF mutation }\end{array}$ & $\begin{array}{l}\text { A. Nivolumab 3mg/kg } \\
\text { Q2wks. } \\
\text { B. Dacarbazine } 1000 \\
\mathrm{mg} / \mathrm{m}^{2} \text { Q3wks. }\end{array}$ & $\begin{array}{c}418 \\
\mathbf{A}=210 \\
\mathbf{B}=208\end{array}$ & $\begin{array}{l}\text { A. NR } \\
\text { B. } 10.8(95 \% \text { CI: } \\
9.3-12.1\end{array}$ & $\begin{array}{c}\text { A. } 5.1(95 \% \mathrm{CI}: \\
3.5-10.8) \\
\text { B. } 2.2(95 \% \mathrm{CI}: \\
2.1-2.4) \\
(\mathrm{HR}=0.43 \\
p<0.001)\end{array}$ & $\begin{array}{c}\text { A. } 40.0 \\
(95 \% \text { CI: } \\
33.3-47.0) \\
\text { B. } 13.9 \\
(9.5-19.4)\end{array}$ & $\begin{array}{l}\text { A. } 7.6 \\
\text { B. } 1.0\end{array}$ & $\begin{array}{l}\text { A. } 11.7 \\
\text { B. } 17.6\end{array}$ & - \\
\hline & $\begin{array}{l}\text { Postow et al. } \\
\text { (2015) [128] } \\
\text { CheckMate } \\
069\end{array}$ & II & $\begin{array}{l}\text { Metastatic melanoma } \\
\text { with no prior treatment }\end{array}$ & $\begin{array}{l}\text { A. Ipilimumab } 3 \mathrm{mg} / \mathrm{kg}+ \\
\text { nivolumab } 1 \mathrm{mg} / \mathrm{kg} \\
\text { Q3wks followed by } \\
\text { nivolumab 3 mg/kg } \\
\text { Q2wks } \\
\text { B. Ipilimumab } 3 \mathrm{mg} / \mathrm{kg}+ \\
\text { placebo Q3wks followed } \\
\text { by placebo Q2wks }\end{array}$ & $\begin{array}{l}142 \\
\text { A. } 95 \\
\text { B. } 47\end{array}$ & - & $\begin{array}{c}\text { A. Not reached } \\
\text { B. } 4.4 \\
\mathrm{HR}=0.4, p<0.001\end{array}$ & $\begin{array}{l}\text { In BRAF } \\
\text { wild-type } \\
\text { group: } \\
\text { A. } 61 \\
\text { B. } 11 \\
p<0.001\end{array}$ & $\begin{array}{l}\text { In BRAF } \\
\text { wild-type } \\
\text { group: } \\
\text { A. } 16 \\
\text { B. } 0\end{array}$ & $\begin{array}{l}\text { A. } 54 \% \\
\text { B. } 20 \%\end{array}$ & $\begin{array}{l}\text { Approved in combination } \\
\text { with ipilimumab for the } \\
\text { treatment of BRAF V600 wild } \\
\text { type unresectable or } \\
\text { metastatic melanoma } \\
\text { (October 2015) }\end{array}$ \\
\hline & $\begin{array}{l}\text { Weber et al. } \\
\text { (2015) [51] } \\
\text { CheckMate } \\
037\end{array}$ & III & Metastatic disease & $\begin{array}{l}\text { A. Nivolumab 3mg/kg } \\
\text { B. Investigator's choice of } \\
\text { chemotherapy }\end{array}$ & $\begin{array}{c}405 \mathbf{A}=272 \\
\mathbf{B}=133\end{array}$ & - & $\begin{array}{l}\text { A. } 4.7(95 \% \text { CI: } \\
\quad 2.3-6.5) \\
\text { B. } 4.2(95 \% \text { CI: } \\
2.1-6.3) \\
(\mathrm{HR}=0.82)\end{array}$ & $\begin{array}{c}\text { A. } 31.7 \\
(95 \% \text { CI: } \\
23.5-40.8) \\
\text { B. } 10.6 \\
(95 \% \text { CI: } \\
3.5-23.1)\end{array}$ & $\begin{array}{l}\text { A. } 3.3 \\
\text { B. } 0.0\end{array}$ & $\begin{array}{l}\text { A. } 9.0 \\
\text { B. } 31.0\end{array}$ & $\begin{array}{c}\text { Unresectable or metastatic } \\
\text { melanoma and disease } \\
\text { progression following } \\
\text { ipilimumab and, if BRAF } \\
\text { V600 mutation positive, a } \\
\text { BRAF inhibitor (December } \\
\text { 2014, based on interim } \\
\text { analysis). }\end{array}$ \\
\hline
\end{tabular}


Table A3. Cont.

\begin{tabular}{|c|c|c|c|c|c|c|c|c|c|c|c|}
\hline Cancer & Study & Phase & Stage of Disease & Treatment Regimen & $\mathbf{N}$ & $\begin{array}{c}\text { Median OS } \\
\text { (Months) }\end{array}$ & $\begin{array}{l}\text { Median PFS } \\
\text { (Months) }\end{array}$ & ORR (\%) & CRR (\%) & $\begin{array}{c}\text { Grade } \\
\text { III/IV AEs } \\
(\%) \\
\end{array}$ & $\begin{array}{l}\text { Resultant FDA-Approved } \\
\text { Indication }\end{array}$ \\
\hline & $\begin{array}{c}\text { Larkin et al. } \\
\text { (2015) [129] } \\
\text { CheckMate } \\
067\end{array}$ & III & $\begin{array}{l}\text { Previously untreated, } \\
\text { stage III (unresectable) } \\
\text { or stage IV disease with } \\
\text { known BRAF V600 } \\
\text { mutation status }\end{array}$ & $\begin{array}{c}\text { A: Nivolumab } 1 \mathrm{mg} / \mathrm{kg}+ \\
\text { ipilimumab 3mg/kg } \\
\text { Q3wks., followed by } \\
\text { nivolumab 3mg/kg } \\
\text { Q2wks. } \\
\text { B: Nivolumab 3mg/kg + } \\
\text { placebo Q2wks.C. } \\
\text { Ipilimumab 2mg/kg + } \\
\text { placebo Q3wks. }\end{array}$ & $\begin{array}{c}945 \\
\mathbf{A}=314 \\
\mathbf{B}=316 \\
\mathbf{C}=315\end{array}$ & - & $\begin{array}{c}\text { A. } 11.5(95 \% \mathrm{CI}: \\
8.9-16.7) \\
\text { B. } 6.9(95 \% \mathrm{CI}: \\
4.3-9.5) \\
\text { C. } 2.9(95 \% \mathrm{CI}: \\
2.8-3.4) \\
\text { (HR= } 0.42 \\
p<0.001 \text { A vs. C; } \\
\text { HR = } 0.57 \\
p<0.001 \text { B vs. C) }\end{array}$ & $\begin{array}{c}\text { A. } 57.6 \\
(95 \% \text { CI } \\
52.0-63.2) \\
\text { B. } 43.7 \\
(95 \% \text { CI: } \\
38.1-49.3) \\
\text { C. } 19(95 \%) \\
\text { CI: } \\
14.9-23.8)\end{array}$ & $\begin{array}{l}\text { A. } 11.5 \\
\text { B. } 8.9 \\
\text { C. } 2.2\end{array}$ & $\begin{array}{l}\text { A. } 55.0 \\
\text { B. } 16.3 \\
\text { C. } 27.3\end{array}$ & $\begin{array}{l}\text { Expanded indication: } \\
\text { unresectable or metastatic } \\
\text { melanoma regardless of } \\
\text { BRAF V600 mutation status } \\
\text { (January 2016) }\end{array}$ \\
\hline & $\begin{array}{l}\text { Weber et al. } \\
(2017)[146] \\
\text { CheckMate } \\
\quad 238\end{array}$ & III & $\begin{array}{l}\text { Completely resected, } \\
\text { advanced disease }\end{array}$ & $\begin{array}{l}\text { A. Nivolumab 3mg/kg } \\
\text { Q2wks. } \\
\text { B. Ipilimumab } 10 \mathrm{mg} / \mathrm{kg} \\
\text { Q3wks. }\end{array}$ & $\begin{array}{c}906 \\
\mathbf{A}=453 \\
\mathbf{B}=453\end{array}$ & - & $\begin{array}{c}\text { 12-month PFS rate: } \\
\text { A. } 70.5 \%(95 \% \mathrm{CI}: \\
66.1-74.5) \\
\text { B. } 60.8 \% \text { (95\% CI: } \\
56.0-65.2) \\
\quad(\mathrm{HR}=0.65 \\
p<0.001) \\
\end{array}$ & - & - & $\begin{array}{l}\text { A. } 14.4 \\
\text { B. } 45.9\end{array}$ & $\begin{array}{l}\text { Expanded indication: } \\
\text { adjuvant treatment for } \\
\text { melanoma with involvement } \\
\text { of lymph nodes or in patients } \\
\text { with metastatic disease who } \\
\text { have undergone complete } \\
\text { resection (December 2017) }\end{array}$ \\
\hline Mesothelioma & $\begin{array}{l}\text { Scherpereel et al. } \\
(2019)[147] \\
\text { IFCT-1501 } \\
\text { MAPS2 }\end{array}$ & II & $\begin{array}{l}\text { Malignant pleural } \\
\text { mesothelioma with } \\
\text { progression after first- or } \\
\text { second-line treatments }\end{array}$ & $\begin{array}{l}\text { A. Nivolumab } 3 \mathrm{mg} / \mathrm{kg} \\
\text { Q2 } 2 \mathrm{wks} \\
\text { B. Nivolumab } 3 \mathrm{mg} / \mathrm{kg} \\
\text { Q2wks + ipilimumab } \\
1 \text { mg } / \mathrm{kg} \text { Q6wks } \\
\end{array}$ & $\begin{array}{c}125 \\
\mathbf{A}=63 \\
\mathbf{B}=62\end{array}$ & $\begin{array}{c}\text { A. } 11.0(95 \% \text { CI: } \\
6.7-17.7) \\
\text { B. } 15.9 \text { (95\% CI: } \\
10.7-\mathrm{NR})\end{array}$ & $\begin{array}{c}\text { A. } 4.0 \text { ( }(95 \% \text { CI: } \\
\text { 2.8-5.7) } \\
\text { B. } 5.6(95 \% \text { CI: } \\
3.1-8.3)\end{array}$ & $\begin{array}{l}\text { A. } 19(95 \%) \\
\text { CI: 8-29) } \\
\text { B. . (28 (95\%) } \\
\text { CI: } 16-49)\end{array}$ & - & $\begin{array}{l}\text { A. } 14 \\
\text { B. } 26\end{array}$ & - \\
\hline \multirow[t]{4}{*}{ NSCLC } & $\begin{array}{c}\text { Rizvi et al. } \\
\text { (2015) [148] } \\
\text { CheckMate } \\
063\end{array}$ & II & Advanced sqNSCLC & $\begin{array}{l}\text { Nivolumab 3mg/kg } \\
\text { Q2wks. }\end{array}$ & 117 & $\begin{array}{l}8.2(95 \% \text { CI: } \\
6.1-10.9)\end{array}$ & $\begin{array}{l}1.9(95 \% \mathrm{CI}: \\
1.8-3.2)\end{array}$ & $\begin{array}{l}14.5(95 \% \\
\text { CI: } \\
8.7-22.2)\end{array}$ & 1.0 & 17.0 & - \\
\hline & $\begin{array}{l}\text { Brahmer et al. } \\
\text { (2015) [52] } \\
\text { CheckMate } \\
017\end{array}$ & III & Advanced sqNSCLC & $\begin{array}{l}\text { A. Nivolumab 3mg/kg } \\
\text { Q2wks. } \\
\text { B. Docetaxel 75mg/m² } \\
\text { Q3wks. }\end{array}$ & $\begin{array}{c}272 \\
\mathbf{A}=135 \\
\mathbf{B}=137\end{array}$ & $\begin{array}{c}\text { A. } 9.2(95 \% \mathrm{CI}: \\
7.3-13.3) \\
\text { B. } 6.0(95 \% \mathrm{CI}: \\
5.1-7.3) \\
(\mathrm{HR}=0.59 \\
p<0.001)\end{array}$ & $\begin{array}{c}\text { A. } 3.5(95 \% \text { CI: } \\
2.1-4.9) \\
\text { B. } 2.8(95 \% \text { CI: } \\
2.1-3.5) \\
(\mathrm{HR}=0.62 \\
p<0.001)\end{array}$ & $\begin{array}{l}\text { A. } 20(95 \%) \\
\text { CI: } 14-28) \\
\text { B. } 9(95 \%) \\
\text { CI } 5-15) \\
(p=0.008)\end{array}$ & $\begin{array}{l}\text { A. } 1.0 \\
\text { B. } 0.0\end{array}$ & $\begin{array}{l}\text { A. } 7.0 \\
\text { B. } 55.0\end{array}$ & $\begin{array}{l}\text { Metastatic sqNSCLC with } \\
\text { progression on or after } \\
\text { platinum-based } \\
\text { chemotherapy (March 2015) }\end{array}$ \\
\hline & $\begin{array}{l}\text { Borghaei et al. } \\
\text { (2015) [53] } \\
\text { CheckMate } \\
057\end{array}$ & III & $\begin{array}{l}\text { Stage IIIB, IV, or } \\
\text { recurrent NsqNSCLC }\end{array}$ & $\begin{array}{l}\text { A. Nivolumab 3mg/kg } \\
\text { Q2wks. } \\
\text { B. Docetaxel 75mg/m² } \\
\text { Q3wks. }\end{array}$ & $\begin{array}{c}582 \\
\mathbf{A}=292 \\
\mathbf{B}=290\end{array}$ & $\begin{array}{c}\text { A. } 12.2(95 \% \mathrm{CI}: \\
\quad 9.7-15.0) \\
\text { B. } 9.4(95 \% \mathrm{CI}: \\
8.1-10.7) \\
(\mathrm{HR}=0.73 \\
p=0.002)\end{array}$ & $\begin{array}{l}\text { A. } 2.3(95 \% \text { CI: } \\
2.2-3.3) \\
\text { B. } 4.2(95 \% \text { CI: } \\
\quad 3.5-4.9)\end{array}$ & $\begin{array}{l}\text { A. } 19(95 \% \\
\text { CI: } 15-24) \\
\text { B. } 12(95 \%) \\
\text { CI: } 9-17) \\
(p=0.02)\end{array}$ & $\begin{array}{l}\text { A. } 1.0 \\
\text { B. }<1.0\end{array}$ & $\begin{array}{l}\text { A. } 10.0 \\
\text { B. } 54.0\end{array}$ & $\begin{array}{l}\text { Expanded indication: } \\
\text { Metastatic NSCLC (squamous } \\
\text { or non-squamous) with } \\
\text { progression on or after } \\
\text { platinum-based therapy } \\
\text { (October 2015) }\end{array}$ \\
\hline & $\begin{array}{l}\text { Carbone etal. } \\
\text { (2017) [149] } \\
\text { CheckMate } \\
026\end{array}$ & III & $\begin{array}{l}\text { Untreated stage IV or } \\
\text { recurrent disease with } \\
\text { PD-L1 expression } \geq 1 \%\end{array}$ & $\begin{array}{l}\text { A. Nivolumab 3mg } / \mathrm{kg} \\
\text { Q2wks. } \\
\text { B. Investigator's choice of } \\
\text { platinum doublet } \\
\text { chemotherapy }\end{array}$ & $\begin{array}{c}541 \\
\mathbf{A}=271 \\
\mathbf{B}=270\end{array}$ & $\begin{array}{l}\text { A. } 14.4(95 \% \mathrm{CI}: \\
11.7-17.4) \\
\text { B. } 13.2(95 \% \mathrm{CI} \\
10.7-17.1) \\
(\mathrm{HR}=1.02)\end{array}$ & $\begin{array}{c}\text { A. } 4.2(95 \% \text { CI: } \\
\text { 3.0-5.6) } \\
\text { B. } 5.9(95 \% \text { CI: } \\
5.4-6.9) \\
(\mathrm{HR}=1.15 \\
p=0.25)\end{array}$ & $\begin{array}{l}\text { A. } 26(95 \% \\
\text { CI: } 20-33) \\
\text { B. } 33(95 \% \\
\text { CI: } 27-40)\end{array}$ & $\begin{array}{l}\text { A. } 2.0 \\
\text { B. }<1.0\end{array}$ & $\begin{array}{l}\text { A. } 18 \\
\text { B. } 51\end{array}$ & - \\
\hline
\end{tabular}


Table A3. Cont.

\begin{tabular}{|c|c|c|c|c|c|c|c|c|c|c|c|}
\hline Cancer & Study & Phase & Stage of Disease & Treatment Regimen & $\mathbf{N}$ & $\begin{array}{l}\text { Median OS } \\
\text { (Months) }\end{array}$ & $\begin{array}{c}\text { Median PFS } \\
\text { (Months) }\end{array}$ & ORR (\%) & CRR (\%) & $\begin{array}{c}\text { Grade } \\
\text { III/IV AEs } \\
(\%)\end{array}$ & $\begin{array}{l}\text { Resultant FDA-Approved } \\
\text { Indication }\end{array}$ \\
\hline & $\begin{array}{l}\text { Vokes et al. } \\
(2018)[54] \\
\text { CheckMate017, } \\
\text { CheckMate } \\
\quad 057\end{array}$ & III & $\begin{array}{l}\text { Advanced NSCLC } \\
\text { (pooled analyses of } \\
\text { CheckMate 017 and } \\
\text { CheckMate 57) }\end{array}$ & $\begin{array}{l}\text { A. Nivolumab 3mg } / \mathrm{kg} \\
\text { Q2wks. } \\
\text { B. Docetaxel 75mg/m² } \\
\text { Q3wks. }\end{array}$ & $\begin{array}{c}\text { Pooled } \\
854 \\
\mathbf{A}=427 \\
\mathbf{B}=427 \\
\text { Liver mets } \\
\mathbf{A}=99 \\
\mathbf{B}=94\end{array}$ & $\begin{array}{c}\text { Pooled analysis:A. } \\
11.1(95 \% \mathrm{CI}: \\
9.2-13.1) \\
\text { B. } 8.1(95 \% \mathrm{CI}: \\
7.2-9.2) \\
(\mathrm{HR}=0.70) \\
\text { Liver mets: } \\
\text { A. } 6.8(95 \% \mathrm{CI}: \\
4.9-10.4) \\
\text { B. } 5.9(95 \% \mathrm{CI}: \\
4.7-7.3) \\
(\mathrm{HR}=0.68)\end{array}$ & $\begin{array}{c}\text { Pooled analysis: } \\
\text { A. } 2.56(95 \% \mathrm{CI}: \\
2.20-3.48) \\
\text { B. } 3.52(95 \% \mathrm{CI}: \\
3.15-4.21) \\
\text { (HR }=0.80) \\
\frac{\text { Liver mets: }}{-}\end{array}$ & $\begin{array}{l}\frac{\text { Ongoing }}{\text { responses }} \\
\frac{\text { at minimum }}{3 \text { yrs. follow up: }} \\
\text { A. } 24 \\
\text { B. } 0\end{array}$ & $\begin{array}{r}\text { A. } 4.0 \\
\text { p: B. } 0.0 \\
\end{array}$ & $\begin{array}{l}\text { Pooled analysis: } \\
\text { A. } 44 \\
\text { B. - } \\
\text { Liver mets: } \\
\text { A. } 8 \\
\text { B. - }\end{array}$ & s: \\
\hline \multirow[t]{3}{*}{ RCC } & $\begin{array}{l}\text { Motzer etal. } \\
\text { (2015) [150] }\end{array}$ & II & Metastatic disease & $\begin{array}{c}\text { A. Nivolumab 0.3mg } / \mathrm{kg} \\
\text { Q3wks. } \\
\text { B. Nivolumab 2mg } / \mathrm{kg} \\
\text { Q3wks. } \\
\text { C. Nivolumab 10mg } / \mathrm{kg} \\
\text { Q3wks. }\end{array}$ & 168 & $\begin{array}{c}\text { A. } 18.2(80 \% \mathrm{CI}: \\
16.2-24.0) \\
\text { B. } 25.0(80 \% \mathrm{CI} \text { : } \\
19.8-28.8) \\
\text { C. } 24.7(95 \% \mathrm{CI} \text { : } \\
15.3-26.0) \\
(\mathrm{HR}=0.8 \text { for B vs. } \\
\text { A; HR }=0.9 \text { for C } \\
\text { vs. A) }\end{array}$ & $\begin{array}{c}\text { A. } 2.7(80 \% \mathrm{CI} \\
1.9-3.0) \\
\text { B. } 4.0(80 \% \mathrm{CI}: \\
2.8-4.2) \\
\text { C. } 4.2(80 \% \mathrm{CI} \text { : } \\
2.8-5.5)\end{array}$ & $\begin{array}{l}\text { A. } 20(80 \% \\
\text { CI } \\
13.4-28.2) \\
\text { B. } 22(80 \% \\
\text { CI: } \\
15.0-31.3) \\
\text { C. } 20(80 \% \\
\text { CI: } \\
13.4-29.1) \\
(p=1.0)\end{array}$ & $\begin{array}{l}\text { A. } 2.0 \\
\text { B. } 2.0 \\
\text { C. } 0.0\end{array}$ & $\begin{array}{l}\text { A. } 5 \\
\text { B. } 17 \\
\text { C. } 13\end{array}$ & - \\
\hline & $\begin{array}{l}\text { Motzer etal. } \\
\text { (2015) [55] } \\
\text { CheckMate } \\
025\end{array}$ & III & Advanced disease & $\begin{array}{l}\text { A. Nivolumab 3mg/kg } \\
\text { Q2wks. } \\
\text { B. Everolimus 10mg QD }\end{array}$ & $\begin{array}{c}821 \\
\mathbf{A}=410 \\
\mathbf{B}=411\end{array}$ & $\begin{array}{l}\text { A. } 25.0 \text { (95\% CI: } \\
\text { 21.7-NE) } \\
\text { B. } 19.6(95 \% \mathrm{CI}: \\
17.6-23.1) \\
(\mathrm{HR}=0.73 \\
p=0.0018) \\
\end{array}$ & $\begin{array}{c}\text { A. } 4.6(95 \% \mathrm{CI}: \\
3.7-5.4) \\
\text { B. } 4.4(95 \% \mathrm{CI} \text { : } \\
3.7-5.5) \\
(\mathrm{HR}=0.88 \\
p=0.11)\end{array}$ & $\begin{array}{c}\text { A. } 25 \\
\text { B. } 5 \\
(p<0.001)\end{array}$ & $\begin{array}{l}\text { A. } 1.0 \\
\text { B. }<1.0\end{array}$ & $\begin{array}{l}\text { A. } 19.0 \\
\text { B. } 37.0\end{array}$ & $\begin{array}{l}\text { Advanced RCC with history } \\
\text { of prior anti-angiogenic } \\
\text { therapy (November 2015) }\end{array}$ \\
\hline & $\begin{array}{l}\text { Motzer et al. } \\
\text { (2018) [39] } \\
\text { CheckMate } \\
214\end{array}$ & III & $\begin{array}{l}\text { Advanced, untreated } \\
\text { disease }\end{array}$ & $\begin{array}{l}\text { A. Nivolumab 3mg/kg + } \\
\text { ipilimumab } 1 \mathrm{mg} / \mathrm{kg} \\
\text { Q2wks. } \\
\text { B. Sunitinib 50mg QD for } \\
4 \text { wks. }\end{array}$ & $\begin{array}{c}1096 \\
\mathbf{A}=550 \\
\mathbf{B}=546\end{array}$ & $\begin{array}{l}\text { A. NR }(95 \% \mathrm{CI} \\
28.2-\mathrm{NE}) \\
\text { B. } 26.0(95 \% \mathrm{CI} \\
22.1-\mathrm{NE}) \\
(\mathrm{HR}=0.63 \\
p \leq 0.001) \\
\end{array}$ & $\begin{array}{c}\text { A. } 11.6(95 \% \mathrm{CI}: \\
\quad 8-7-15.5) \\
\text { B. } 8.4(95 \% \mathrm{CI} \text { : } \\
\quad 7.0-10.8) \\
(\mathrm{HR}=0.82 \\
\quad p=0.03) \\
\end{array}$ & $\begin{array}{l}\text { A. } 42(95 \% \\
\text { CI: } 37-47) \\
\text { B. } 27(95 \% \\
\text { CI: } 22-31) \\
(p<0.001)\end{array}$ & $\begin{array}{l}\text { A. } 9 \\
\text { B. } 1\end{array}$ & $\begin{array}{l}\text { A: } 46 \\
\text { B: } 63\end{array}$ & $\begin{array}{c}\text { Expanded indication: } \\
\text { Combination } \\
\text { nivolumab/ipilimumab for } \\
\text { intermediate- or poor-risk, } \\
\text { previously untreated } \\
\text { advanced RCC (April 2018) }\end{array}$ \\
\hline SCLC & $\begin{array}{l}\text { Antonia et al. } \\
\text { (2016) [56] } \\
\text { CheckMate } \\
032\end{array}$ & $\mathrm{I} / \mathrm{II}$ & $\begin{array}{c}\text { Limited-stage or } \\
\text { extensive stage disease, } \\
\text { after failing } \\
\text { platinum-based } \\
\text { chemotherapy and one } \\
\text { other line of treatment }\end{array}$ & 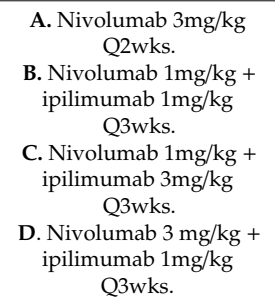 & $\begin{array}{c}216 \\
\mathbf{A}=98 \\
\mathbf{B}=3 \\
\mathbf{C}=61 \\
\mathbf{D}=54\end{array}$ & $\begin{array}{c}\text { A. } 4.4 \text { ( } 95 \% \text { CI: } \\
\text { 3.0-9.3) } \\
\text { B. - } \\
\text { C. } 4.4(95 \% \text { CI: } \\
\text { 3.6-18.0) } \\
\text { D. } 6.0(95 \% \text { CI: } \\
\quad 3.6-11.0)\end{array}$ & $\begin{array}{l}\text { A. } 1.4(95 \% \text { CI: } \\
\text { 1.4-1.9) } \\
\text { B. }- \\
\text { C. } 2.6(95 \% \text { CI: } \\
\text { 1.4-4.1) } \\
\text { D. } 1.4(1.3-2.2)\end{array}$ & $\begin{array}{l}\text { A. } 10(95 \% \\
\text { CI: } 5-18) \\
\text { B. } 33(95 \% \\
\text { CI: } \\
0.9-91.0) \\
\text { C. } 23(95 \% \\
\text { CI: } 13-36) \\
\text { D. } 19(95 \% \\
\text { CI: } 9-31)\end{array}$ & $\begin{array}{l}\text { A. } 0.0 \\
\text { B. }- \\
\text { C. } 2.0 \\
\text { D. } 0.0\end{array}$ & $\begin{array}{l}\text { A. } 13.0 \\
\text { B. } 0.0 \\
\text { C. } 30.0 \\
\text { D. } 19.0\end{array}$ & $\begin{array}{l}\text { Metastatic SCLC with } \\
\text { proggession after } \\
\text { platinum-based } \\
\text { chemotherapy and at least } \\
\text { one other line of therapy } \\
\text { (August 2018) }\end{array}$ \\
\hline
\end{tabular}

ASCT $=$ autologous stem cell transplant, $\mathrm{BV}=$ brentuximab vedotin, $\mathrm{CI}=$ confidence interval, $\mathrm{CRC}=$ colorectal cancer, $\mathrm{dMMR}=\mathrm{DNA}$ mismatch repair-deficient, $\mathrm{GBM}=$ glioblastoma multiforme, GEJ = gastroesophageal junction, HCC = hepatocellular carcinoma, HL = Hodgkin lymphoma, HNSCC = head and neck squamous cell carcinoma, HR = hazard ratio, MSI-H = microsatellite instability-high, MTX = methotrexate, NE = not estimable, NR = not reached, NSCLC = non-small cell lung cancer, NsqNSCLC $=$ non-squamous non-small cell lung cancer, RCC = renal cell carcinoma, SCC $=$ squamous cell carcinoma, sqNSCLC $=$ squamous non-small cell lung cancer. 
Table A4. Select clinical data for pembrolizumab.

\begin{tabular}{|c|c|c|c|c|c|c|c|c|c|c|c|}
\hline Cancer & Study & Phase & Stage of Disease & Treatment Regimen & $\mathbf{N}$ & $\begin{array}{l}\text { Median OS } \\
\text { (Months) }\end{array}$ & $\begin{array}{l}\text { Median PFS } \\
\text { (Months) }\end{array}$ & ORR (\%) & CRR (\%) & $\begin{array}{c}\text { Grade } \\
\text { III/IV AEs } \\
(\%)\end{array}$ & $\begin{array}{l}\text { Resultant FDA-Approved } \\
\text { Indication }\end{array}$ \\
\hline \multirow[t]{2}{*}{ Bladder cancer } & $\begin{array}{l}\text { Bellmunt et al. } \\
\text { (2017) [151] } \\
\text { KEYNOTE-045 }\end{array}$ & III & $\begin{array}{l}\text { Metastatic urothelial } \\
\text { carcinoma that } \\
\text { recurred or } \\
\text { progressed after } \\
\text { platinum } \\
\text { chemotherapy }\end{array}$ & $\begin{array}{l}\text { A. Pembrolizumab } \\
\text { 200mg Q3wks. } \\
\text { B. Investigator's } \\
\text { choice of paclitaxel, } \\
\text { docetaxel, or } \\
\text { vinflunine Q3wks. }\end{array}$ & $\begin{array}{l}542 \\
\text { A. } 270 \\
\text { B. } 272\end{array}$ & $\begin{array}{c}\text { A. } 10.3 \\
\quad \text { B. } 7.4 \\
(\mathrm{HR}=0.73 \\
p=0.002)\end{array}$ & $\begin{array}{c}\text { A. } 2.1 \\
\text { B. } 3.3 \\
(\mathrm{HR}=0.98, p=0.42)\end{array}$ & $\begin{array}{l}\text { A. } 21 \\
\text { B. } 11\end{array}$ & - & $\begin{array}{l}\text { A. } 15.0 \\
\text { B. } 49.4\end{array}$ & $\begin{array}{l}\text { Locally advanced or } \\
\text { metastatic urothelial } \\
\text { carcinoma with progression } \\
\text { during or following } \\
\text { platinum-containing } \\
\text { chemotherapy or within } 12 \\
\text { months of neoadjuvant or } \\
\text { adjuvant treatment with } \\
\text { platinum-containing } \\
\text { chemotherapy (May 2017) }\end{array}$ \\
\hline & $\begin{array}{c}\text { Balar et al. } \\
\text { (2017) [152] } \\
\text { KEYNOTE-052, } \\
\text { with update } \\
\text { from } \\
\text { O'Donnell et al. } \\
\text { (2019) [153] }\end{array}$ & II & $\begin{array}{l}\text { Advanced urothelial } \\
\text { carcinoma ineligible } \\
\text { for platin }\end{array}$ & $\begin{array}{l}\text { Pembrolizumab } \\
\text { 200mg Q3wks. }\end{array}$ & $\begin{array}{l}\quad 370 \\
\text { CPS }<10.251 \\
\text { CPS } \geq 10.110\end{array}$ & $\begin{array}{l}\text { Combined: } 11.3 \\
\text { CPS }<10: 9.7 \\
\underline{\operatorname{CPS} \geq 10: 18.5}\end{array}$ & $\frac{\text { Combined: } 2}{\text { months }}$ & $\begin{array}{l}\text { Combined: } 28.4 \\
\text { CPS }<10: 20.0 \\
\text { CPS } \geq 10: 10.0\end{array}$ & $\frac{\text { Combined: }}{9.0}$ & 21 & $\begin{array}{l}\text { Expanded indication: locally } \\
\text { advanced or metastatic } \\
\text { urothelial carcinoma who are } \\
\text { not eligible for } \\
\text { cisplatin-containing therapy } \\
\text { and whose tumors express } \\
\text { PD-L1 (CPS } \geq 10 \text { ), or in } \\
\text { patients who are not eligible } \\
\text { for any platinum-containing } \\
\text { chemotherapy regardless of } \\
\text { PD-L1 status (June 2018) }\end{array}$ \\
\hline CSCC & $\begin{array}{c}\text { Chung et al. } \\
\text { (2019) [57] } \\
\text { KEYNOTE-158 }\end{array}$ & II & $\begin{array}{c}\text { Recurrent or } \\
\text { metastatic advanced } \\
\text { disease that has } \\
\text { failed one or more } \\
\text { lines of } \\
\text { chemotherapy }\end{array}$ & $\begin{array}{l}\text { Pembrolizumab } \\
\text { 200mg Q3wks. }\end{array}$ & $\begin{array}{c}98 \\
\text { CPS }<1.16 \\
\text { CPS } \geq 1.82\end{array}$ & $\begin{array}{l}\text { Combined: } 9.4 \\
\underline{\text { CPS } \geq 1: 11}\end{array}$ & $\begin{array}{l}\text { Combined: } 2.1 \\
\underline{\underline{\text { CPS } \geq 1: 2.1}}\end{array}$ & $\begin{array}{l}\text { Combined: } 12.2 \\
\underline{\text { CPS }<1: 1.0} \\
\underline{\text { PS } \geq 1: 14.6}\end{array}$ & $\frac{\text { Combined: }}{3}$ & 12.2 & $\begin{array}{l}\text { Second-line for recurrent or } \\
\text { metastatic cervical cancer } \\
\text { with CPS } \geq 1 \text { (June 2018) }\end{array}$ \\
\hline $\begin{array}{l}\text { Endometrial } \\
\text { Carcinoma }\end{array}$ & $\begin{array}{c}\text { Makker et al. } \\
(2019)[94] \\
\text { KEYNOTE-146 }\end{array}$ & II & $\begin{array}{c}\text { Metastatic } \\
\text { endometrial cancer } \\
\text { that had progressed } \\
\text { following at least } \\
\text { one prior systemic } \\
\text { therapy }\end{array}$ & $\begin{array}{c}\text { Pembrolizumab } \\
\text { 200mg IV Q3wks + } \\
\text { lenvatinib 20mg PO } \\
\text { QD }\end{array}$ & $\begin{array}{l}108,94 \text { of which } \\
\text { were not MSI-H } \\
\text { or dMMR }\end{array}$ & - & - & 38.3 & 10.6 & 52 & $\begin{array}{l}\text { Approved in combination } \\
\text { with lenvatinib for the } \\
\text { treatment of patients with } \\
\text { advanced endometrial } \\
\text { carcinoma that is not MSI-H } \\
\text { or dMMR, or who have } \\
\text { disease progression following } \\
\text { prior systemic therapy and } \\
\text { are not candidates for } \\
\text { curative surgery or radiation } \\
\text { (September 2019) }\end{array}$ \\
\hline $\begin{array}{l}\text { Esophageal } \\
\text { cancer }\end{array}$ & $\begin{array}{l}\text { Shah et al. } \\
\text { (2019) [154] } \\
\text { KEYNOTE-180 }\end{array}$ & II & $\begin{array}{c}\text { Advanced, } \\
\text { metastatic } \\
\text { esophageal cancer } \\
\text { that progressed after } \\
2 \text { or more lines of } \\
\text { therapy }\end{array}$ & $\begin{array}{l}\text { Pembrolizumab } \\
\text { 200mg Q3wks. }\end{array}$ & $\begin{array}{l}121 \\
\text { ESCC. } 63 \\
\text { EAC. } 58\end{array}$ & $\begin{array}{c}\text { ESCC: } 6.8 \\
\text { EAC: } 3.9 \\
\text { CPS < 10: } 5.4 \\
\text { CPS } \geq 10: 6.4 \\
(\mathrm{HR}=0.64)\end{array}$ & $\begin{array}{l}\text { ESCC: } 2.1 \\
\text { EAC: } 1.9 \\
\text { CPS < 10: } 2.0 \\
\text { CPS } \geq 10: 2.0 \\
(\mathrm{HR}=0.66)\end{array}$ & $\begin{array}{c}\text { ESCC: } 14.3 \\
\text { EAC: } 5.2 \\
\text { CPS }<10: 6.3 \\
\text { CPS } \geq 10: 13.8\end{array}$ & $\begin{array}{l}\text { ESCC: } 0.0 \\
\text { EAC: } 0.0\end{array}$ & $\frac{\text { Combined: }}{11.60}$ & $\begin{array}{l}\text { Recurrent locally advanced or } \\
\text { metastatic squamous cell } \\
\text { carcinoma of the esophagus } \\
\text { whose tumors express PD-L1 } \\
\text { (CPS } \geq 10 \text { ) (July 2019) }\end{array}$ \\
\hline
\end{tabular}


Table A4. Cont.

\begin{tabular}{|c|c|c|c|c|c|c|c|c|c|c|c|}
\hline Cancer & Study & Phase & Stage of Disease & Treatment Regimen & $\mathbf{N}$ & $\begin{array}{l}\text { Median OS } \\
\text { (Months) }\end{array}$ & $\begin{array}{c}\text { Median PFS } \\
\text { (Months) }\end{array}$ & ORR (\%) & CRR (\%) & $\begin{array}{c}\text { Grade } \\
\text { III/IV AEs } \\
(\%)\end{array}$ & $\begin{array}{l}\text { Resultant FDA-Approved } \\
\text { Indication }\end{array}$ \\
\hline & $\begin{array}{l}\text { Kojima et al. } \\
(2019)[155] \\
\text { KEYNOTE-181 }\end{array}$ & III & $\begin{array}{l}\text { Locally advanced or } \\
\text { metastatic disease }\end{array}$ & $\begin{array}{l}\text { A. Pembrolizumab } \\
\text { 200mg Q3wks, } \\
\text { B. Investigator's } \\
\text { choice of paclitaxel, } \\
\text { docetaxel, or } \\
\text { irinotecan }\end{array}$ & $\begin{array}{c}628 \\
\text { ESCC. } 401 \\
\text { CPS } \geq 10.222\end{array}$ & $\begin{array}{c}\text { ESCC: } \\
\text { A. } 8.2 \\
\text { B. } 7.1 \\
(H R=0.78, \\
p=0.0095) \\
\text { CPS } \geq 10: \\
\text { A. } 9.3 \\
\text { B. } 6.7 \\
(H R=0.69, \\
p=0.0074)\end{array}$ & - & - & - & $\begin{array}{l}\text { A. } 18 \\
\text { B. } 41\end{array}$ & $\begin{array}{l}\text { As above, with Shah et al. } \\
\text { (2019) }\end{array}$ \\
\hline $\begin{array}{l}\text { Gastric or GEJ } \\
\text { adenocarcinoma }\end{array}$ & $\begin{array}{c}\text { Fuchs et al. } \\
\text { (2018) [58] } \\
\text { KEYNOTE-059 }\end{array}$ & II & $\begin{array}{c}\text { Gastric/GEJ } \\
\text { adenocarcinoma } \\
\text { previously treated } \\
\text { with } 2 \text { or more } \\
\text { systemic therapies } \\
\text { including } \\
\text { fluoropyrimidine } \\
\text { and platinum, } \\
\text { and HER2/neu if } \\
\text { indicated }\end{array}$ & $\begin{array}{l}\text { Pembrolizumab } \\
\text { 200mg Q3wks. }\end{array}$ & 259 & 5.6 & 2.0 & $\begin{array}{c}\text { Overall: } 11.6 \\
\text { PD-L1+: } 15.5 \\
\text { PD-L1-: } 6.4\end{array}$ & 2.4 & 17.8 & $\begin{array}{l}\text { Second-line therapy for } \\
\text { patients with recurrent, } \\
\text { locally advanced, or } \\
\text { metastatic esophageal } \\
\text { squamous cell carcinoma } \\
\text { (September 2017) }\end{array}$ \\
\hline HCC & $\begin{array}{c}\text { Zhu et al. } \\
\text { (2018) [62] } \\
\text { KEYNOTE-224 }\end{array}$ & II & $\begin{array}{l}\text { HCC with prior } \\
\text { treatment with } \\
\text { sorafenib }\end{array}$ & $\begin{array}{l}\text { Pembrolizumab } \\
\text { 200mg Q3wks. }\end{array}$ & 104 & 12.9 & 4.8 & 17 & 1 & 24 & $\begin{array}{l}\text { Monotherapy for HCC that } \\
\text { had been previously treated } \\
\text { with sorafenib (November } \\
\text { 2018) }\end{array}$ \\
\hline HL & $\begin{array}{l}\text { Chen et al. } \\
\text { (2017) [63] } \\
\text { KEYNOTE-087 }\end{array}$ & & $\begin{array}{l}\text { Recurrent or } \\
\text { relapsed cHL }\end{array}$ & $\begin{array}{c}\text { Pembrolizumab } 200 \\
\text { mg Q3wks. in } 3 \\
\text { cohorts: } \\
\text { A. After ASCT and } \\
\text { BV } \\
\text { B. After salvage } \\
\text { chemotherapy and } \\
\text { BV } \\
\text { C. After ASCT only }\end{array}$ & $\begin{array}{l}210 \\
\text { A. } 69 \\
\text { B. } 81 \\
\text { C. } 60\end{array}$ & $\begin{array}{c}\text { NR } \\
\text { 24-month OS rate: } \\
\text { A. } 92.5 \\
\text { B. } 90.6 \\
\text { C. } 89.4\end{array}$ & $\begin{array}{l}\text { Overall: } 13.7 \\
\text { A. } 16.4 \\
\text { B. } 11.1 \\
\text { C. } 19.4\end{array}$ & $\begin{array}{l}\text { Overall: } 71.9 \\
\text { A. } 76.8 \\
\text { B. } 66.7 \\
\text { C. } 73.3\end{array}$ & $\begin{array}{l}\text { A. } 26.1 \\
\text { B. } 25.9 \\
\text { C. } 31.7\end{array}$ & 11.9 & $\begin{array}{l}\text { Refractory or relapsed cHL } \\
\text { after three or more lines of } \\
\text { prior therapy (March 2017) }\end{array}$ \\
\hline HNSCC & $\begin{array}{l}\text { Seiwert et al. } \\
(2016)[59] \\
\text { KEYNOTE-012 }\end{array}$ & $\mathrm{Ib}$ & $\begin{array}{l}\text { Recurrent or } \\
\text { metastatic disease } \\
\text { with PD-L1 positive } \\
\text { status }\end{array}$ & $\begin{array}{l}\text { Pembrolizumab } \\
10 \mathrm{mg} / \mathrm{kg} \text { Q2wks. }\end{array}$ & 60 & 13 & 2 & 18 & 2 & 17 & $\begin{array}{c}\text { Metastatic or recurrent } \\
\text { HNSCC with disease } \\
\text { progression on or after } \\
\text { platinum therapy (August } \\
\text { 2016) }\end{array}$ \\
\hline & $\begin{array}{l}\text { Burtness et al. } \\
(2018) \text { [61] } \\
\text { KEYNOTE-048 }\end{array}$ & III & $\begin{array}{l}\text { Locally incurable } \\
\text { recurrent or } \\
\text { metastatic disease } \\
\text { and no prior } \\
\text { systemic therapy }\end{array}$ & $\begin{array}{l}\text { A. Pembrolizumab } \\
\text { 200mg Q3wks. } \\
\text { B. Pembrolizumab } \\
\text { 200mg Q3wks. }+6 \\
\text { cycles of platinum } \\
\text { therapy } \\
\text { C. Cetuximab + } 6 \\
\text { cycles of platinum + } \\
\text { FU }\end{array}$ & $\begin{array}{l}882 \\
\text { A. } 301 \\
\text { B. } 281 \\
\text { C. } 300\end{array}$ & $\begin{array}{c}\text { Overall: } \\
\text { B. } 11.5 \\
\text { C. } 10.7 \\
\text { (HR }=0.83, \\
p=0.0199) \\
\text { CPS } 220: \\
\text { B. } 14.7 \\
\text { C. } 11 \\
(H R=0.60, \\
p=0.0004) \\
\text { CPS } \geq 1: \\
\text { B. } 13.6 \\
\text { C. } 10.4 \\
(H R=0.65, \\
p<0.0001)\end{array}$ & $\begin{array}{c}\text { NR } \\
\text { Overall: } \\
\text { HR }=1.29 \\
\text { CPS } \geq 20: \\
\text { HR }=0.76, p=0.5 \\
\begin{array}{c}\text { No further PFS } \\
\text { analysis done }\end{array}\end{array}$ & $\begin{array}{c}\text { Overall: } \\
\text { B. } 16.9 \\
\text { C. } 36.0 \\
\text { CPS } 220: \\
\text { B. } 42.9 \\
\text { C. } 38.2 \\
\text { CPS } \geq 1: \\
\text { B. } 36.4 \\
\text { C. } 35.7\end{array}$ & - & $\begin{array}{l}\text { A. } 54.7 \\
\text { B. } 85.1 \\
\text { C. } 83.3\end{array}$ & - \\
\hline
\end{tabular}


Table A4. Cont.

\begin{tabular}{|c|c|c|c|c|c|c|c|c|c|c|c|}
\hline Cancer & Study & Phase & Stage of Disease & Treatment Regimen & $\mathbf{N}$ & $\begin{array}{c}\text { Median OS } \\
\text { (Months) }\end{array}$ & $\begin{array}{l}\text { Median PFS } \\
\text { (Months) }\end{array}$ & ORR (\%) & CRR $(\%)$ & $\begin{array}{c}\text { Grade } \\
\text { III/IV AEs } \\
(\%)\end{array}$ & $\begin{array}{l}\text { Resultant FDA-Approved } \\
\text { Indication }\end{array}$ \\
\hline & $\begin{array}{l}\text { Rischin et al. } \\
2019[156] \\
\text { (KEYNOTE-048 } \\
\text { final analysis) }\end{array}$ & III & $\begin{array}{l}\text { Locally incurable } \\
\text { recurrent or } \\
\text { metastatic disease } \\
\text { and no prior } \\
\text { systemic therapy }\end{array}$ & $\begin{array}{l}\text { A. Pembrolizumab } \\
\text { 200mg Q3wks. } \\
\text { B. Pembrolizumab } \\
\text { 200mg Q3wks. }+6 \\
\text { cycles of platinum } \\
\text { therapy } \\
\text { C. Cetuximab + } 6 \\
\text { cycles of platinum + } \\
\text { FU }\end{array}$ & $\begin{array}{l}882 \\
\text { A. } 301 \\
\text { B. } 281 \\
\text { C. } 300\end{array}$ & $\begin{array}{c}\text { CPS } \geq 20: \\
\text { A. } 14.9 \\
\text { C. } 10.7 \\
(\mathrm{HR}=0.61 \\
p=0.0015) \\
\mathrm{CPS} \geq 1: \\
\text { A. } 12.3 \\
\text { C. } 10.3 \\
(\mathrm{HR}=0.65, \\
p<0.0001) \\
\text { Overall } \\
\text { B. } 13.0 \\
\text { C. } 10.7 \\
(\mathrm{HR}=0.77, \\
p=0.0067)\end{array}$ & $\begin{array}{c}\mathrm{CPS} \geq 20: \\
\text { A. 3.4 } \\
\text { C. } 5.0 \\
(\mathrm{HR}=0.99) \\
\text { CPS } \geq 1: \\
\text { A. } 3.2 \\
\text { C. } 5.0 \\
(\text { HR }=1.15) \\
\text { Overall } \\
\text { B. } 4.9 \\
\text { C. } 5.1 \\
(\mathrm{HR}=0.92 \\
p=0.3394)\end{array}$ & $\begin{array}{l}\frac{\mathrm{CPS} \geq 20:}{\mathrm{A} .23} \\
\text { C. } 36 \\
\text { CPS } \geq 1 \text { : } \\
\text { A. } 19 \\
\text { C. } 35 \\
\text { Overall } \\
\text { B. } 36 \\
\text { C. } 36\end{array}$ & $\begin{array}{l}\frac{\text { CPS } \geq 20:}{\text { A. } 8} \\
\text { C. } 3 \\
\text { CPS } \geq 1 \text { : } \\
\text { A. } 5 \\
\text { C. } 13 \\
\text { Overall } \\
\text { B. } 6 \\
\text { C. } 3\end{array}$ & $\begin{array}{l}\text { A. } 54.7 \\
\text { B. } 85.1 \\
\text { C. } 83.3\end{array}$ & $\begin{array}{l}\text { First line treatment of patients } \\
\text { with metastatic or } \\
\text { unresectable recurrent } \\
\text { HNSCC, as monotherapy in } \\
\text { patients whose tumors } \\
\text { express PD-L1 or in } \\
\text { combination with platinum } \\
\text { and fluorouracil (June 2019) }\end{array}$ \\
\hline & $\begin{array}{c}\text { Cohen et al. } \\
\text { (2019) [60] } \\
\text { KEYNOTE-040 }\end{array}$ & III & $\begin{array}{c}\text { Recurrent or } \\
\text { metastatic disease }\end{array}$ & $\begin{array}{l}\text { A. Pembrolizumab } \\
\text { 10mg/kg Q3wks. } \\
\text { B. Investigator's } \\
\text { choice of MTX, } \\
\text { docetaxel, cetuximab }\end{array}$ & $\begin{array}{c}495 \\
\text { A. } 247 \\
\text { B. } 248\end{array}$ & $\begin{array}{l}\frac{\mathrm{CPS} \geq 1:}{\text { A. } 8.7} \\
\text { B. } 7.1\end{array}$ & $\begin{array}{c}\text { A. } 2.1 \\
\text { B. } 2.3 \\
(\mathrm{HR}=0.95 \\
p=0.030)\end{array}$ & $\begin{array}{l}\text { A. } 14.6 \\
\text { B. } 10.1\end{array}$ & - & $\begin{array}{l}\text { A. } 13 \\
\text { B. } 36\end{array}$ & $\begin{array}{l}\text { Expanded indication: } \\
\text { combination with platinum } \\
\text { and fluorouracil (FU) as } \\
\text { first-line treatment of } \\
\text { metastatic or unresectable, } \\
\text { recurrent HNSCC, and as } \\
\text { single agent for patients } \\
\text { whose tumors express PD-L1 } \\
\text { and CPS } \geq 1 \text { (June 2019) }\end{array}$ \\
\hline MCC & $\begin{array}{l}\text { Nghiem et al. } \\
\text { (2019) [69] } \\
\text { KEYNOTE-017 }\end{array}$ & II & $\begin{array}{c}\text { Recurrent locally } \\
\text { advanced Merkel } \\
\text { Cell Carcinoma or } \\
\text { metastatic MCC } \\
\text { with no prior } \\
\text { therapy }\end{array}$ & $\begin{array}{l}\text { Pembrolizumab } \\
2 \mathrm{mg} / \mathrm{kg} \text { Q3wks. }\end{array}$ & 50 & NR & 16.8 & 56 & - & 28 & $\begin{array}{l}\text { Treatment of adult and } \\
\text { pediatric patients with } \\
\text { recurrent locally advanced or } \\
\text { metastatic MCC (December } \\
\text { 2018) }\end{array}$ \\
\hline Melanoma & $\begin{array}{l}\text { Robert et al. } \\
(2014) \text { [65] } \\
\text { KEYNOTE-001 }\end{array}$ & I & $\begin{array}{c}\text { Unresectable or } \\
\text { metastatic disease } \\
\text { with progression } \\
\text { following } \\
\text { ipilimumab and, if } \\
\text { BRAF V600 } \\
\text { mutation positive, } \\
\text { BRAF inhibitor }\end{array}$ & $\begin{array}{l}\text { A. Pembrolizumab } \\
2 \mathrm{mg} / \mathrm{kg} \text { Q3wks. } \\
\text { B. Pembrolizumab } \\
\text { 10mg/kg Q3wks. }\end{array}$ & $\begin{array}{c}173 \\
\text { A. } 89 \\
\text { B. } 84\end{array}$ & $\begin{array}{c}\text { 12-month survival rate: } \\
\text { A. } 58 \\
\text { B. } 63 \\
(\mathrm{HR}=1.09)\end{array}$ & $\begin{array}{c}\text { A. } 5.1 \\
\text { B. } 3.2 \\
\text { (HR }=0.84)\end{array}$ & $\begin{array}{l}\text { Combined: } 26 \\
\text { A. } 26 \\
\text { B. } 26 \\
(p=0.96)\end{array}$ & $\begin{array}{l}\text { A. } 1 \\
\text { B. } 1\end{array}$ & $\begin{array}{l}\text { Combined: } \\
12 \\
\text { A. } 15 \\
\text { B. } 8\end{array}$ & $\begin{array}{l}\text { Unresectable or metastatic } \\
\text { melanoma with disease } \\
\text { progression following } \\
\text { ipilimumab and, if BRAF } \\
\text { V600 mutation positive, BRAF } \\
\text { inhibitor (September 2014) }\end{array}$ \\
\hline & $\begin{array}{c}\text { Ribas et al. } \\
\text { (2015) [66] } \\
\text { KEYNOTE-002 }\end{array}$ & II & $\begin{array}{c}\text { Advanced } \\
\text { melanoma following } \\
\text { ipilimumab and, if } \\
\text { BRAF V600 positive, } \\
\text { BRAF inhibitor }\end{array}$ & $\begin{array}{l}\text { A. Pembrolizumab } \\
\text { 2mg/kg Q3wks.B. } \\
\text { Pembrolizumab } \\
\text { 10m/kg Q3wks.B. } \\
\text { Investigator's choice } \\
\text { of paclitaxel + } \\
\text { carboplatin, } \\
\text { paclitaxel, } \\
\text { carboplatin, } \\
\text { dacarbazine, or } \\
\text { temozolomide }\end{array}$ & $\begin{array}{l}540 \\
\text { A. } 180 \\
\text { B. } 181 \\
\text { C. } 179\end{array}$ & - & $\begin{array}{c}\text { A. } 2.9 \\
\text { B. } 2.9 \\
\text { C. } 2.7 \\
\text { (HR }=0.57 \\
p<0.0001 \text { A vs. C; } \\
\mathrm{HR}=0.50, p<0.0001 \\
\text { B vs. C) }\end{array}$ & $\begin{array}{l}\text { A. } 38 \\
\text { B. } 46 \\
\text { C. } 8\end{array}$ & $\begin{array}{l}\text { A. } 2 \\
\text { B. } 3 \\
\text { C. } 0\end{array}$ & $\begin{array}{l}\text { A. } 11 \\
\text { B. } 14 \\
\text { C. } 26\end{array}$ & - \\
\hline
\end{tabular}


Table A4. Cont.

\begin{tabular}{|c|c|c|c|c|c|c|c|c|c|c|c|}
\hline Cancer & Study & Phase & Stage of Disease & Treatment Regimen & $\mathbf{N}$ & $\begin{array}{l}\text { Median OS } \\
\text { (Months) }\end{array}$ & $\begin{array}{l}\text { Median PFS } \\
\text { (Months) }\end{array}$ & ORR (\%) & CRR (\%) & $\begin{array}{l}\text { Grade } \\
\text { III/IV AEs } \\
(\%)\end{array}$ & $\begin{array}{l}\text { Resultant FDA-Approved } \\
\text { Indication }\end{array}$ \\
\hline & $\begin{array}{c}\text { Robert et al. } \\
(2015)[67] \\
\text { KEYNOTE-006 }\end{array}$ & III & $\begin{array}{l}\text { Stage III or IV } \\
\text { melanoma with no } \\
\text { more than } 1 \text { prior } \\
\text { treatment }\end{array}$ & $\begin{array}{c}\text { A. Pembrolizumab } \\
10 \mathrm{mg} / \mathrm{kg} \text { Q2wks. } \\
\text { B. Pembrolizumab } \\
\text { 10mg/kg Q3wks.C. } \\
\text { Ipilimumab 3mg } / \mathrm{kg} \\
\text { Q3wks. }\end{array}$ & $\begin{array}{l}834 \\
\text { A. } 279 \\
\text { B. } 277 \\
\text { C. } 278\end{array}$ & $\begin{array}{c}\text { NR for all groups } \\
\text { 12-month OS rate: } \\
\text { A. } 74.1 \\
\text { B. } 68.1 \\
\text { C. } 58.2 \\
\text { (HR }=0.63 \\
p<0.0005 \text { A vs. C; } \\
\text { HR }=0.69 \\
p=0.0036 \text { B vs. C) }\end{array}$ & $\begin{array}{c}\text { A. } 5.5 \\
\text { B. } 4.1 \\
\text { C. } 2.8 \\
(\mathrm{HR}=0.58, p<0.001 \\
\text { A vs. C; } \mathrm{HR}=0.58 \\
p<0.001 \text { B } \mathrm{\text {vs. }} \text { C) }\end{array}$ & $\begin{array}{c}\text { A. } 33.7 \\
\text { B. } 32.9 \\
\text { C. } 11.9 \\
(p<0.001 \text { A vs. C; } \\
p<0.001 \text { B vs. C) }\end{array}$ & $\begin{array}{l}\text { A. } 5.0 \\
\text { B. } 6.1 \\
\text { C. } 1.4\end{array}$ & $\begin{array}{l}\text { A. } 13.3 \\
\text { B. } 10.1 \\
\text { C. } 19.9\end{array}$ & $\begin{array}{l}\text { Expanded indication: } \\
\text { first-line treatment of } \\
\text { unresectable or metastatic } \\
\text { melanoma (December 2015) }\end{array}$ \\
\hline & $\begin{array}{l}\text { Eggermont et al. } \\
\text { (2018) [68] } \\
\text { KEYNOTE-054 }\end{array}$ & III & $\begin{array}{l}\text { Completely resected } \\
\text { stage III disease }\end{array}$ & $\begin{array}{l}\text { A. Pembrolizumab } \\
\text { 200mg Q3wks. } \\
\text { B. Placebo }\end{array}$ & $\begin{array}{l}1019 \\
\text { A. } 514 \\
\text { B. } 505\end{array}$ & - & $\begin{array}{c}\frac{\text { Median recurrence- }}{\text { free survival }} \\
\text { A. NR } \\
\text { B. } 20.4 \\
(\text { HR }=0.57 \\
p<0.001) \\
\end{array}$ & $\begin{array}{l}\frac{\text { Recurrence rate: }}{\text { A. } 26} \\
\text { B. } 43\end{array}$ & - & $\begin{array}{l}\text { A. } 14.7 \\
\text { B. } 3.4\end{array}$ & $\begin{array}{l}\text { Expanded indication: } \\
\text { adjuvant treatment of } \\
\text { melanoma following } \\
\text { complete resection (February } \\
\text { 2019) }\end{array}$ \\
\hline \multirow[t]{2}{*}{$\begin{array}{l}\text { MSI-H or } \\
\text { dMMR }\end{array}$} & $\begin{array}{c}\text { Le et al. } \\
\text { (2018) [157] } \\
\text { KEYNOTE-164 }\end{array}$ & II & $\begin{array}{c}\text { Metastatic CRC with } \\
>2 \text { prior treatments } \\
\text { including FU, } \\
\text { oxaliplatin, } \\
\text { and irinotecan }+/- \\
\text { anti-VEGF/EGFR } \\
\text { mAb }\end{array}$ & $\begin{array}{l}\text { Pembrolizumab } \\
\text { 200mg Q3wks. }\end{array}$ & 61 & NR & 4.1 & 32 & 3.1 & 11 & $\begin{array}{c}\text { Adult and pediatric patients } \\
\text { with MSI-H or dMMR solid } \\
\text { tumors that have progressed } \\
\text { with no other treatment } \\
\text { alternatives or colorectal } \\
\text { cancer that has progressed } \\
\text { after fluoropyrimidine, } \\
\text { oxaliplatin, and irinotecan } \\
\text { (May 2017) }\end{array}$ \\
\hline & $\begin{array}{l}\text { KEYNOTE-016, } \\
018,028,158 \text { [70] }\end{array}$ & & $\begin{array}{l}\text { MSI-H/dMMR CRC, } \\
\text { gastric, bladder, } \\
\text { breast, biliary, } \\
\text { endometrial, } \\
\text { esophageal cancer } \\
\end{array}$ & $\begin{array}{c}\text { Pembrolizumab at } \\
\text { varying doses }\end{array}$ & $\begin{array}{c}149 \\
\text { CRC }=90 \\
\text { Other types }=59\end{array}$ & - & - & $\begin{array}{c}\text { Combined: } 39.6 \\
\text { CRC: } 36 \\
\underline{\text { Other: } 46}\end{array}$ & 7.4 & - & - \\
\hline NPC & $\begin{array}{c}\text { Hsu et al. } \\
\text { (2017) [158] } \\
\text { KEYNOTE-028 }\end{array}$ & $\mathrm{Ib}$ & $\begin{array}{l}\text { Unresectable or } \\
\text { metastatic disease, } \\
\text { failure on standard } \\
\text { therapy, PD-L1 } \\
\text { expression in } 1 \% \text { or } \\
\text { more of tumor cells }\end{array}$ & $\begin{array}{l}\text { Pembrolizumab } \\
10 \mathrm{mg} / \mathrm{kg} \text { Q2wks }\end{array}$ & 27 & $\begin{array}{l}16.5 \text { (95\% CI: } \\
10.1-\mathrm{NR})\end{array}$ & 3.7 (95\% CI: 2.1-13.4) & $\begin{array}{l}25.9 \text { (95\% CI: } \\
11.1-46.3)\end{array}$ & 0 & 29.6 & - \\
\hline NSCLC & $\begin{array}{c}\text { Garon et al. } \\
\text { (2015) [64] } \\
\text { KEYNOTE-001 }\end{array}$ & I & Advanced disease & $\begin{array}{l}\text { A. Pembrolizumab } \\
2 \mathrm{mg} / \mathrm{kg} \text { Q3wks. } \\
\text { B. Pembrolizumab } \\
\text { 10mg/kg Q3wks. } \\
\text { C. Pembrolizumab } \\
10 \mathrm{mg} / \mathrm{kg} \text { Q2wks. }\end{array}$ & $\begin{array}{c}495 \\
\text { Prior } \\
\text { treatment }=394 \\
\text { No prior } \\
\text { treatment }=101\end{array}$ & $\begin{array}{c}\text { Combined: } 12.0 \\
\text { Prior treatment: } 9.3 \\
\text { No prior treatment: } \\
16.2\end{array}$ & $\begin{array}{c}\text { Combined: } 3.7 \\
\frac{\text { Prior treatment: }}{3.0} \\
\text { No prior treatment: } \\
6.0\end{array}$ & $\begin{array}{l}\text { Combined: } 19.4 \\
\frac{\text { Prior treatment: }}{18.0} \\
\frac{\text { No prior treatment: }}{24.8}\end{array}$ & - & Combined: 9.5 & $\begin{array}{l}\text { Metastatic NSCLC with } \\
\text { PD-L1 expression and disease } \\
\text { progression on or after } \\
\text { platinum therapy; those with } \\
\text { EGFR or ALK tumor } \\
\text { mutations should have } \\
\text { disease progression on } \\
\text { FDA-approved therapy for } \\
\text { these mutations prior to } \\
\text { pembrolizumab (October } \\
\text { 2015) }\end{array}$ \\
\hline
\end{tabular}


Table A4. Cont.

\begin{tabular}{|c|c|c|c|c|c|c|c|c|c|c|c|}
\hline \multirow[t]{6}{*}{ Cancer } & Study & Phase & Stage of Disease & Treatment Regimen & $\mathbf{N}$ & $\begin{array}{l}\text { Median OS } \\
\text { (Months) }\end{array}$ & $\begin{array}{l}\text { Median PFS } \\
\text { (Months) }\end{array}$ & ORR (\%) & CRR (\%) & $\begin{array}{l}\text { Grade } \\
\text { III/IV AEs } \\
(\%)\end{array}$ & $\begin{array}{l}\text { Resultant FDA-Approved } \\
\text { Indication }\end{array}$ \\
\hline & $\begin{array}{l}\text { Reck et al. } \\
\text { (2016) [159] } \\
\text { KEYNOTE-024 } \\
\text { interim analysis }\end{array}$ & III & $\begin{array}{l}\text { Previously untreated } \\
\text { NSCLC with } \\
\text { TPS } \geq 50 \% \text { and no } \\
\text { EGFR or ALK } \\
\text { mutations }\end{array}$ & $\begin{array}{l}\text { A. Pembrolizumab } \\
\text { 200mg Q3wks } \\
\text { B. Investigator's } \\
\text { choice platinum } \\
\text { therapy }\end{array}$ & $\begin{array}{c}305 \\
\mathbf{A}=154 \\
\mathbf{B}=151\end{array}$ & $\begin{array}{c}\text { MOS not reached, } \\
\text { overall survival } \\
\text { greater in group A } \\
\text { with } \mathrm{HR}=0.6 \text {, } \\
p=0.005)\end{array}$ & $\begin{array}{c}\text { A. } 10.3 \\
\text { B. } 6.0 \\
(\mathrm{HR}=0.5, p<0.001)\end{array}$ & $\begin{array}{l}\text { A. } 44.8 \\
\text { B. } 27.8\end{array}$ & - & $\begin{array}{l}\text { A. } 26.6 \\
\text { B. } 53.3\end{array}$ & $\begin{array}{l}\text { First line therapy metastatic } \\
\text { NSCLC with TPS }>50 \% \text { and } \\
\text { no EGFR, ALK mutations } \\
\text { (October 2016) }\end{array}$ \\
\hline & $\begin{array}{l}\text { Herbst et al. } \\
\text { (2016) [160] } \\
\text { KEYNOTE-010 }\end{array}$ & $\mathrm{II} / \mathrm{III}$ & $\begin{array}{l}\text { Previously treated } \\
\text { metastatic NSCLC } \\
\quad \text { with TPS }>1 \%\end{array}$ & $\begin{array}{c}\text { A. Pembrolizumab } \\
\text { 2mg/kg Q3wks.B. } \\
\text { Pembrolizumab } \\
\text { 10mg/kg Q3wks.C. } \\
\text { Docetaxel 75mg/m² } \\
\text { Q3wks. }\end{array}$ & $\begin{array}{c}1034 \\
\mathbf{A}=345 \\
\mathbf{B}=346 \\
\mathbf{C}=343\end{array}$ & $\begin{array}{c}\text { A. } 10.4 \\
\text { B. } 12.7 \\
\text { C. } 8.5(\mathrm{HR}=0.71 \\
p \leq 0.001 \text { A vs. C; } \\
\mathrm{HR}=0.61, \mathrm{p} \leq 0.001 \\
\text { B vs. C) }\end{array}$ & $\begin{array}{c}\text { A. } 3.9 \\
\text { B. } 4.0 \\
\text { C. } 4.0 \\
(\mathrm{HR}=0.88, p=0.068 \\
\text { A vs. C; } \mathrm{HR}=0.79, \\
p=0.005 \text { B vs. C) }\end{array}$ & $\begin{array}{l}\text { A. } 18 \\
\text { B. } 19 \\
\text { C. } 9\end{array}$ & - & $\begin{array}{l}\text { A. } 13 \\
\text { B. } 16 \\
\text { C. } 35\end{array}$ & $\begin{array}{l}\text { Expanded indication: } \\
\text { second-line therapy for } \\
\text { metastatic NSCLC with TPS } \\
>1 \% \text { following disease } \\
\text { progression on or after } \\
\text { platinum chemotherapy } \\
\text { (October 2016) }\end{array}$ \\
\hline & $\begin{array}{c}\text { Gandhi et al. } \\
\text { (2018) [161] } \\
\text { KEYNOTE-189 }\end{array}$ & III & $\begin{array}{l}\text { Previously untreated } \\
\text { metastatic } \\
\text { NsqNSCLC without } \\
\text { ALK or EGFR } \\
\text { mutations }\end{array}$ & $\begin{array}{c}\text { A. Pemetrexed and } \\
\text { platinum-based } \\
\text { therapy }+ \\
\text { pembrolizumab } \\
\text { Q3wks.B. Placebo } \\
\text { Q2wks. } 4 \text { cycles, } \\
\text { then } \\
\text { pembrolizumab/placebo } \\
\text { for up to } 35 \text { cycles + } \\
\text { pemetrexed } \\
\end{array}$ & $\begin{array}{c}616 \\
\mathbf{A}=405 \\
\mathbf{B}=202\end{array}$ & $\begin{array}{c}\text { A. NR } \\
\text { B. } 11.3 \\
(\mathrm{HR}=0.49 \\
p \leq 0.0001)\end{array}$ & $\begin{array}{c}\text { A. } 8.8 \\
\text { B. } 4.8 \\
(\mathrm{HR}=0.52 \\
p \leq 0.0001)\end{array}$ & $\begin{array}{l}\text { A. } 47.6 \\
\text { B. } 18.9\end{array}$ & $\begin{array}{l}\text { A. } 0.5 \\
\text { B. } 0.5\end{array}$ & $\begin{array}{l}\text { A. } 67.2 \\
\text { B. } 65.8\end{array}$ & $\begin{array}{l}\text { Expanded indication: } \\
\text { first-line therapy in } \\
\text { combination with } \\
\text { platinum-based } \\
\text { chemotherapy and } \\
\text { pemetrexed for metastatic } \\
\text { NsqNSCLC without EGFR or } \\
\text { ALK genomic aberrations } \\
\text { (May 2018) }\end{array}$ \\
\hline & $\begin{array}{c}\text { Paz-Ares et al. } \\
\text { (2018) [162] } \\
\text { KEYNOTE-407 }\end{array}$ & III & $\begin{array}{l}\text { Untreated metastatic } \\
\text { squamous disease }\end{array}$ & $\begin{array}{c}\text { A. Pembrolizumab } \\
\text { 200mg + carboplatin } \\
+ \\
\text { paclitaxel/nab-paclitaxel } \\
\text { for first } 4 \text { cyclesB. } \\
\text { saline placebo }+ \\
\text { carboplatin and } \\
\text { paclitaxel or } \\
\text { nab-paclitaxel for } \\
\text { first } 4 \text { cycles }\end{array}$ & $\begin{array}{c}559 \\
\mathbf{A}=278 \\
\mathbf{B}=281\end{array}$ & $\begin{array}{c}\text { A. } 15.9 \\
\text { B. } 11.3 \\
(\mathrm{HR}=0.64 \\
p=0.0017)\end{array}$ & $\begin{array}{c}\text { A. } 6.4 \\
\text { B. } 4.8 \\
(\mathrm{HR}=0.56, \\
p \leq 0.0001)\end{array}$ & $\begin{array}{l}\text { A. } 58 \\
\text { B. } 35\end{array}$ & - & $\begin{array}{l}\text { A. } 69.8 \\
\text { B. } 68.2\end{array}$ & $\begin{array}{l}\text { Expanded indication: } \\
\text { first-line therapy in } \\
\text { combination with carboplatin } \\
\text { and paclitaxel/nab-paclitaxel } \\
\text { for metastatic sqNSCLC } \\
\text { (October 2018) }\end{array}$ \\
\hline & $\begin{array}{c}\text { Reck et al. } \\
\text { (2019) [163] } \\
\text { KEYNOTE-024 }\end{array}$ & III & $\begin{array}{l}\text { Metastatic disease } \\
\text { with TPS }>50 \% \\
\text { without ALK or } \\
\text { EGFR mutations }\end{array}$ & $\begin{array}{l}\text { A. Pembrolizumab } \\
\text { 200mg Q3wks. } \\
\text { B. Investigator's } \\
\text { choice of } \\
\text { platinum-based } \\
\text { chemo } \\
\text { (platinum-based } \\
\text { therapy + } \\
\text { paclitaxel/pemetrexed/gen }\end{array}$ & $\begin{array}{l}\quad 305 \\
\mathbf{A}=154 \\
\mathbf{B}=151 \\
\end{array}$ & $\begin{array}{c}\text { A. } 30 \\
\text { B. } 14.2 \\
(\mathrm{HR}=0.60 \\
p=0.005)\end{array}$ & $\begin{array}{c}\text { A. } 10.3 \\
\text { B. } 6.0 \\
(\mathrm{HR}=0.50 \\
p \leq 0.001)\end{array}$ & $\begin{array}{l}\text { A. } 44.8 \\
\text { B. } 27.8\end{array}$ & $\begin{array}{l}\text { A. } 4 \\
\text { B. } 1\end{array}$ & $\begin{array}{l}\text { A. } 26.6 \\
\text { B. } 53.3\end{array}$ & $\begin{array}{l}\text { Expanded indication: } \\
\text { first-line treatment for stage } \\
\text { III/IV NSCLC that is not } \\
\text { amenable to surgical resection } \\
\text { or definitive chemoradiation } \\
\text { with TPS } \geq 1 \% \text { and without } \\
\text { EGFR or ALK mutations } \\
\text { (April 2019) }\end{array}$ \\
\hline PMBCL & $\begin{array}{c}\text { Armand et al. } \\
(2018) \text { [71] } \\
\text { KEYNOTE-170 }\end{array}$ & II & $\begin{array}{l}\text { Refractory or } \\
\text { relapsed disease } \\
\text { after or who were } \\
\text { ineligible for ASCT } \\
\mathrm{w} / \geq 2 \text { lines of } \\
\text { therapy }\end{array}$ & $\begin{array}{l}\text { Pembrolizumab } \\
\text { 200mg Q3wks. }\end{array}$ & 53 & NR & 5.5 & 45 & 11 & 26 & $\begin{array}{l}\text { Approved as monotherapy in } \\
\text { refractory or relapsed PMBCL } \\
\text { after or who were ineligible } \\
\text { for ASCT } w / \geq 2 \text { lines of } \\
\text { therapy (June 2018) }\end{array}$ \\
\hline
\end{tabular}


Table A4. Cont.

\begin{tabular}{|c|c|c|c|c|c|c|c|c|c|c|c|}
\hline Cancer & Study & Phase & Stage of Disease & Treatment Regimen & $\mathrm{N}$ & $\begin{array}{l}\text { Median OS } \\
\text { (Months) }\end{array}$ & $\begin{array}{l}\text { Median PFS } \\
\text { (Months) }\end{array}$ & ORR (\%) & CRR (\%) & $\begin{array}{c}\text { Grade } \\
\text { III/IV AEs } \\
(\%)\end{array}$ & $\begin{array}{l}\text { Resultant FDA-Approved } \\
\text { Indication }\end{array}$ \\
\hline RCC & $\begin{array}{c}\text { Rini et al. } \\
\text { (2019) [73] } \\
\text { KEYNOTE-426 }\end{array}$ & I & $\begin{array}{l}\text { Preciously untreated } \\
\text { advanced disease }\end{array}$ & $\begin{array}{c}\text { A. 200mg } \\
\text { Pembrolizumab } \\
\text { Q3wks. + axitinib } \\
\text { 5mg BIDB. Sunitinib } \\
\text { 50mg daily for first } 4 \\
\text { weeks of each } \\
\text { 6-week cycle }\end{array}$ & $\begin{array}{c}861 \\
\mathbf{A}=432 \\
\mathbf{B}=423\end{array}$ & $\begin{array}{c}\mathrm{NR}(\mathrm{HR}=0.53 \\
\quad p<0.0001)\end{array}$ & $\begin{array}{c}\text { A. } 15.1 \\
\text { B. } 11.1 \\
(\mathrm{HR}=0.69 \\
p<0.001)\end{array}$ & $\begin{array}{c}\text { A. } 59.3 \\
\text { B. } 35.7 \\
(p<0.001)\end{array}$ & - & $\begin{array}{l}\text { A. } 75.8 \\
\text { B. } 70.6\end{array}$ & $\begin{array}{l}\text { First-line therapy in } \\
\text { combination with axitinib for } \\
\text { patients with advanced RCC } \\
\text { (April 2019) }\end{array}$ \\
\hline \multirow[t]{2}{*}{ SCLC } & $\begin{array}{c}\text { Ott et al. } \\
\text { (2017) [164] } \\
\text { KEYNOTE-028 }\end{array}$ & I & $\begin{array}{l}\text { SCLC or other lung } \\
\text { neuroendocrine } \\
\text { tumor with } \\
\text { PD-L1 } 1 \geq 1 \% \text { that has } \\
\text { previously failed } \\
\text { platinum therapy } \\
\text { plus etoposide }\end{array}$ & $\begin{array}{l}\text { Pembrolizumab } \\
\text { 10mg/kg Q2wks. }\end{array}$ & 24 & 9.7 & 1.9 & 33.3 & 4.2 & 33 & - \\
\hline & $\begin{array}{c}\text { Chung et al. } \\
\text { (2019) [165] } \\
\text { KEYNOTE-158 }\end{array}$ & II & $\begin{array}{l}\text { Unresectable or } \\
\text { metastatic disease } \\
\text { that has failed } 2 \\
\text { prior lines of therapy }\end{array}$ & $\begin{array}{l}\text { Pembrolizumab } \\
\text { 200mg Q3wks. }\end{array}$ & 98 & 9.4 & 2.1 & 12.20 & 3 & 12.20 & $\begin{array}{l}\text { Second-line therapy for SCLC } \\
\text { or other lung neuroendocrine } \\
\text { tumor that has failed previous } \\
\text { platinum-based therapy and } \\
\text { one other prior line of therapy } \\
\text { (June 2019) }\end{array}$ \\
\hline
\end{tabular}

$\mathrm{ALK}=$ anaplastic lymphoma kinase, $\mathrm{ASCT}=$ autologous stem cell transplant, $\mathrm{BV}=$ brentuximab vedotin, $\mathrm{cHL}=$ classical Hodgkin lymphoma, $\mathrm{CI}=$ confidence interval, $\mathrm{CPS}=\mathrm{combined}$ positive score (CPS), CSCC = cervical squamous cell carcinoma, EAC = esophageal adenocarcinoma, EGFR = epidermal growth factor receptor, ESCC $=$ esophageal squamous cell carcinoma, $\mathrm{FU}=$ fluorouracil, GEJ = gastroesophageal junction, $\mathrm{HL}=$ Hodgkin lymphoma, $\mathrm{HNSCC}=$ head and neck squamous cell carcinoma, $\mathrm{HR}=$ hazard ratio, $\mathrm{MCC}=\mathrm{Merkel}$ cell carcinoma, MTX = methotrexate, NPC = nasopharyngeal carcinoma, NR = not reached, NSCLC = non-small cell lung cancer, NsqNSCLC = non-squamous non-small cell lung cancer, PMBCL = primary mediastinal B-cell lymphoma, RCC = renal cell carcinoma, SCLC = small cell lung cancer, sqNSCLC = squamous non-small cell lung cancer, TPS = tissue polypeptide-specific antigen. 
Table A5. Clinical data for PD-L1 inhibitors.

\begin{tabular}{|c|c|c|c|c|c|c|c|c|c|c|c|c|}
\hline Therapy & Cancer & Study & Phase & $\begin{array}{l}\text { Stage of } \\
\text { Disease }\end{array}$ & Treatment Regimen & $\mathbf{N}$ & $\begin{array}{l}\text { Median OS } \\
\text { (months) }\end{array}$ & $\begin{array}{l}\text { Median PFS } \\
\text { (months) }\end{array}$ & ORR (\%) & CRR (\%) & $\begin{array}{l}\text { Grade } \\
\text { III/IV } \\
\text { AEs (\%) }\end{array}$ & $\begin{array}{l}\text { Resultant FDA-Approved } \\
\text { Indication }\end{array}$ \\
\hline \multirow[t]{4}{*}{ Atezolizumab } & \multirow[t]{3}{*}{ Bladder cancer } & $\begin{array}{l}\text { Rosenberg et al. } \\
\text { (2016) [74] } \\
\text { IMvigor210 }\end{array}$ & II & $\begin{array}{l}\text { Inoperable } \\
\text { locally } \\
\text { advanced or } \\
\text { metastatic } \\
\text { urothelial } \\
\text { carcinoma }\end{array}$ & $\begin{array}{l}\text { Atezolizumab } \\
\text { 1200mg Q3wks. }\end{array}$ & 310 & $\begin{array}{l}11.4 \text { (95\% CI: } \\
\text { 9.0-NE) }\end{array}$ & $\begin{array}{l}2.1 \text { (95\% CI: } \\
2.1-2.1)\end{array}$ & $\begin{array}{c}\text { Overall: } \\
15 \text { (95\% CI: } 11-19) \\
\text { IC2/3: 26 (95\% CI: } \\
18-36) \\
\text { IC1/2/3: } \\
18(95 \% \text { CI: } 13-24)\end{array}$ & 15 & 16 & $\begin{array}{l}\text { Locally advanced or metastatic } \\
\text { urothelial carcinoma with disease } \\
\text { progression during or following } \\
\text { platinum-containing chemotherapy or } \\
\text { disease progression within } 12 \text { months } \\
\text { of neoadjuvant or adjuvant treatment } \\
\text { with platinum containing } \\
\text { chemotherapy (May 2016) }\end{array}$ \\
\hline & & $\begin{array}{l}\text { Balar et al. } \\
\text { (2017) [166] } \\
\text { IMvigor210 }\end{array}$ & II & $\begin{array}{l}\text { Locally } \\
\text { advanced or } \\
\text { metastatic } \\
\text { urothelial } \\
\text { carcinoma who } \\
\text { were cisplatin } \\
\text { ineligible } \\
\end{array}$ & $\begin{array}{l}\text { Atezolizumab } \\
\text { 1200mg Q3wks. }\end{array}$ & 119 & $\begin{array}{l}15.9 \text { (95\% CI: } \\
9.0-\mathrm{NE})\end{array}$ & $\begin{array}{l}2.7 \text { (95\% CI: } \\
2.1-4.2)\end{array}$ & 23 (95\% CI:16-31) & 9 & 19 & $\begin{array}{l}\text { Expanded indication: first line } \\
\text { treatment for locally advanced or } \\
\text { metastatic urothelial carcinoma not } \\
\text { eligible for cisplatin-containing } \\
\text { chemotherapy or within } 12 \text { months of } \\
\text { neoadjuvant or adjuvant } \\
\text { chemotherapy (April 2017) }\end{array}$ \\
\hline & & $\begin{array}{l}\text { Powles et al. } \\
\text { (2018) [75] } \\
\text { IMvigor211 }\end{array}$ & III & $\begin{array}{l}\text { Metastatic } \\
\text { urothelial } \\
\text { carcinoma with } \\
\text { progression } \\
\text { after platinum } \\
\text { therapy }\end{array}$ & $\begin{array}{l}\text { A. Atezolizumab } \\
\text { 1200mg Q3wks. } \\
\text { B. Investigator's } \\
\text { choice of vinflunine, } \\
\text { paclitaxel, or } \\
\text { docetaxel }\end{array}$ & $\begin{array}{l}931 \\
\mathbf{A}=467 \\
\mathbf{B}=464\end{array}$ & $\begin{array}{c}\text { A. } 11.1 \text { (95\% CI: } \\
8.6-15.5) \\
\text { B. } 10.6(95 \% \text { CI: } \\
8.4-12.2) \\
(\mathrm{HR}=0.87 \\
p=0.412)\end{array}$ & $\begin{array}{c}\text { A. } 2.1(95 \% \text { CI: } \\
1.2-2.2) \\
\text { B. } 4.0(95 \% \text { CI: } \\
\quad 3.4-4.2)\end{array}$ & $\begin{array}{l}\text { A. } 13.4 \text { (95\% CI: } \\
10.5-16.9) \\
\text { B. } 13.4 \text { (95\% CI: } \\
\quad 10.5-16.9)\end{array}$ & $\begin{array}{l}\text { A. } 3.0 \\
\text { B. } 3.0\end{array}$ & $\begin{array}{l}\text { A. } 20 \\
\text { B. } 43\end{array}$ & $\begin{array}{l}\text { Expanded indication: locally } \\
\text { advanced or metastatic urothelial } \\
\text { carcinoma ineligible for } \\
\text { cisplatin-containing therapy and } \\
\text { tumor expressing PD-L1 in at least 5\% } \\
\text { of tumor area, or not eligible for } \\
\text { platinum-containing therapy } \\
\text { regardless of PD-L1 status (June 2018) }\end{array}$ \\
\hline & $\begin{array}{c}\text { Breast cancer, } \\
\text { triple-negative }\end{array}$ & $\begin{array}{l}\text { Schmid et al. } \\
\text { (2018) [76] } \\
\text { IMpassion130 }\end{array}$ & III & $\begin{array}{l}\text { Unresectable } \\
\text { locally } \\
\text { advanced } \\
\text { TNBC }\end{array}$ & $\begin{array}{l}\text { A. Atezolizumab } \\
\text { 840mg Q2wks. }+ \\
\text { nab-paclitaxel } \\
100 \mathrm{mg} / \mathrm{m}^{2} \text { on days } 1, \\
\text { 8, and } 15 \text { of every } \\
\text { 28-day cycle } \\
\text { B. Placebo }+ \\
\text { nab-paclitaxel at } \\
\text { dosing above }\end{array}$ & $\begin{array}{l}902 \\
\mathbf{A}=451 \\
\mathbf{B}=451\end{array}$ & $\begin{array}{c}\text { A. } 21.3 \\
\text { B. } 17.6 \\
(\mathrm{HR}=0.84 \\
p=0.08)\end{array}$ & $\begin{array}{c}\text { A. } 7.2 \\
\text { B. } 5.5 \\
(\mathrm{HR}=0.80 \\
p=0.002)\end{array}$ & $\begin{array}{l}\text { A. } 56.0(95 \% \text { CI: } \\
51.3-60.6) \\
\text { B. } 45.9(95 \% \text { CI: } \\
41.2-50.6) \\
(p=0.002)\end{array}$ & $\begin{array}{l}\text { A. } 7.1 \\
\text { B. } 1.6\end{array}$ & $\begin{array}{l}\text { A. } 48.7 \\
\text { B. } 42.2\end{array}$ & $\begin{array}{l}\text { Unresectable or metastatic TNBC with } \\
\text { at least } 1 \% \text { tumor expression of PD-L1 } \\
\text { (March 2019) }\end{array}$ \\
\hline
\end{tabular}


Table A5. Cont.

\begin{tabular}{|c|c|c|c|c|c|c|c|c|c|c|c|c|}
\hline Therapy & Cancer & Study & Phase & $\begin{array}{l}\text { Stage of } \\
\text { Disease }\end{array}$ & Treatment Regimen & $\mathbf{N}$ & $\begin{array}{l}\text { Median OS } \\
\text { (months) }\end{array}$ & $\begin{array}{l}\text { Median PFS } \\
\text { (months) }\end{array}$ & ORR (\%) & CRR (\%) & $\begin{array}{l}\text { Grade } \\
\text { III/IV } \\
\text { AEs (\%) }\end{array}$ & $\begin{array}{l}\text { Resultant FDA-Approved } \\
\text { Indication }\end{array}$ \\
\hline & Melanoma & $\begin{array}{l}\text { Sullivan et al. } \\
\text { (2019) [167] }\end{array}$ & $\mathrm{Ib}$ & $\begin{array}{l}\text { BRAF V600 } \\
\text { mutated } \\
\text { metastatic } \\
\text { disease }\end{array}$ & $\begin{array}{l}\text { A. Atezolizumab + } \\
\text { vemurafenib } 720 \mathrm{mg} \\
\text { BID } \\
\text { B. Vemurafenib } 960 \\
\text { mg BID x49d } \\
\text { followed by } \\
\text { vemurafenib 720 mg } \\
\text { BID x7d, followed by } \\
\text { vemurafenib + } \\
\text { atezolizumab } 720 \mathrm{mg} \\
\text { BID } \\
\text { C. Vemurafenib } 960 \\
\text { mg BII } 21 \mathrm{~d} \text {, } \\
\text { followed by } \\
\text { vemurafenib } 720 \mathrm{mg} \\
\text { BID x7d, followed by } \\
\text { vemurafenib }+ \\
\text { atezolizumab } 720 \mathrm{mg} \\
\text { BID } \\
\text { D. Vemurafenib } 960 \\
\text { mg BID }+ \\
\text { cobimetinib } 60 \mathrm{mg} \\
\text { QD x21, followed } \\
\text { by vemurafenib 720 } \\
\text { mg BID x7d, } \\
\text { followed by } \\
\text { vemurafenib }+ \\
\text { atezolizumab } 720 \mathrm{mg} \\
\text { BID + cobimetinib } 60 \\
\text { mg QD }\end{array}$ & $\begin{array}{l}\text { A. } 3 \\
\text { B. } 8 \\
\text { C. } 6 \\
\text { D. } 39\end{array}$ & 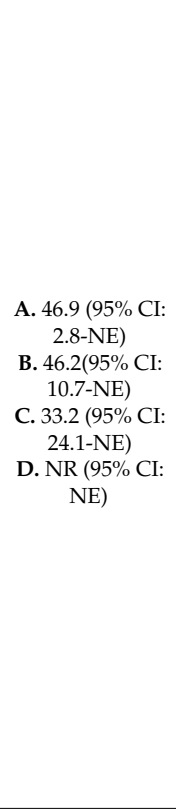 & 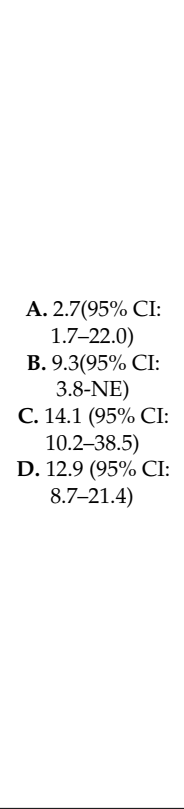 & $\begin{array}{c}\text { A. } 33.3 \text { (95\% CI: } \\
\text {.8-90.6) } \\
\text { B. } 75.0(95 \% \text { CI: } \\
34.9-96.8) \\
\text { C. } 100(95 \% \text { CI: } \\
54.1-100.0) \\
\text { D. } 71.8(95 \% \text { CI: } \\
55.1-85.0)\end{array}$ & $\begin{array}{c}\text { A. } 33.3 \\
(95 \% \text { CI: } \\
0.8-90.6) \\
\text { B. } 12.5 \\
(95 \% \text { CI: } \\
0.3-52.7) \\
\text { C. } 16.7 \\
(95 \% \text { CI: } \\
0.4-64.1) \\
\text { D. 20.5 } \\
(95 \% \text { CI: } \\
9.3-36.5)\end{array}$ & $\begin{array}{l}\text { A. } 100 \\
\text { B. } 87.5 \\
\text { C. } 83.3 \\
\text { D. } 66.7\end{array}$ & - \\
\hline & \multirow[t]{2}{*}{ NSCLC } & $\begin{array}{l}\text { Fehrenbacher et } \\
\text { (2016) [77] } \\
\text { POPLAR }\end{array}$ & tal. & $\begin{array}{l}\text { Previously } \\
\text { treated } \\
\text { advanced or } \\
\text { metastatic } \\
\text { disease }\end{array}$ & $\begin{array}{l}\text { A. Atezolizumab } \\
\text { 1200mg Q3wks. } \\
\text { B. Docetaxel } \\
75 \mathrm{mg} / \mathrm{m}^{2} \text { Q3wks. }\end{array}$ & $\begin{array}{c}287 \\
\mathbf{A}=144 \\
\mathbf{B}=143\end{array}$ & $\begin{array}{c}\text { A. } 12.6 \\
\text { B. } 9.7 \\
(\mathrm{HR}=0.73)\end{array}$ & $\begin{array}{c}\text { A. } 2.7 \\
\text { B. } 3.0 \\
(\mathrm{HR}=0.94 \\
p=0.645)\end{array}$ & 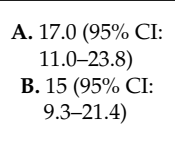 & - & $\begin{array}{l}\text { A. } 11 \\
\text { B. } 39\end{array}$ & $\begin{array}{l}\text { Metastatic NSCLC that had } \\
\text { progressed during or following } \\
\text { platinum-based therapy (October } \\
\text { 2016) }\end{array}$ \\
\hline & & $\begin{array}{l}\text { Rittmeyer et al. } \\
\text { (2017) [168] } \\
\text { OAK }\end{array}$ & III & $\begin{array}{l}\text { Previously } \\
\text { treated stage } \\
\text { IIIB or IV } \\
\text { disease }\end{array}$ & $\begin{array}{l}\text { A. Atezolizumab } \\
\text { 1200mg Q3wks. } \\
\text { B. Docetaxel } \\
75 \mathrm{mg} / \mathrm{m}^{2} \mathrm{Q} 3 \mathrm{wks} \text {. }\end{array}$ & $\begin{array}{c}850 \\
\mathbf{A}=425 \\
\mathbf{B}=425\end{array}$ & $\begin{array}{l}\text { A. } 13.8 \text { (95\% CI: } \\
11.8-15.7 \\
\text { B. } 9.6 \text { ( } 95 \% \text { CI: } \\
8.6-11.2) \\
(\mathrm{HR}=0.74 \\
p=0.0004)\end{array}$ & $\begin{array}{c}\text { A. } 2.8(95 \% \text { CI: } \\
\text { 2.6-4.0) } \\
\text { B. } 4.0(95 \% \text { CI: } \\
2.9-4.3) \\
(\mathrm{HR}=0.91 \\
p=0.38)\end{array}$ & $\begin{array}{l}\text { A. } 58 \\
\text { B. } 57\end{array}$ & $\begin{array}{l}\text { A. } 1 \\
\text { B. }<1\end{array}$ & $\begin{array}{l}\text { A. } 37 \\
\text { B. } 54\end{array}$ & $\begin{array}{l}\text { As above for Fehrenbacher et al. } \\
\text { (2016) }\end{array}$ \\
\hline
\end{tabular}


Table A5. Cont.

\begin{tabular}{|c|c|c|c|c|c|c|c|c|c|c|c|c|}
\hline Therapy & Cancer & Study & Phase & $\begin{array}{l}\text { Stage of } \\
\text { Disease }\end{array}$ & Treatment Regimen & $\mathbf{N}$ & $\begin{array}{l}\text { Median OS } \\
\text { (months) }\end{array}$ & $\begin{array}{l}\text { Median PFS } \\
\text { (months) }\end{array}$ & ORR (\%) & CRR (\%) & $\begin{array}{l}\text { Grade } \\
\text { III/IV } \\
\text { AEs }(\%)\end{array}$ & $\begin{array}{l}\text { Resultant FDA-Approved } \\
\text { Indication }\end{array}$ \\
\hline & & $\begin{array}{l}\text { Socinski et al. } \\
\text { (2018) [78] } \\
\text { IMpower150 }\end{array}$ & III & $\begin{array}{c}\text { Metastatic } \\
\text { NsqNSCLC } \\
\text { without prior } \\
\text { therapy }\end{array}$ & $\begin{array}{c}\text { A. Atezolizumab } \\
1200 \mathrm{mg}+ \\
\text { bevacizumab } \\
15 \mathrm{mg} / \mathrm{kg}+\text { paclitaxel } \\
200 \mathrm{mg} / \mathrm{m}^{2} \text { (or } \\
175 \mathrm{mg} / \mathrm{m}^{2} \text { for Asian } \\
\text { patients) + } \\
\text { carboplatin } \\
6 \mathrm{mg} / \mathrm{mL} / \mathrm{min} \\
\text { B. Carboplatin }+ \\
\text { paclitaxel }+ \\
\text { bevacizumab at } \\
\text { doses above }\end{array}$ & $\begin{array}{c}800 \\
\mathbf{A}=400 \\
\mathbf{B}=400\end{array}$ & $\begin{array}{c}\text { A. } 19.2 \\
\text { B. } 14.7 \\
(\mathrm{HR}=0.78 \\
p=0.02)\end{array}$ & $\begin{array}{c}\text { A. } 8.3 \\
\text { B. } 6.8 \\
(\mathrm{HR}=0.62 \\
p<0.001)\end{array}$ & $\begin{array}{l}\text { A. } 63.5(95 \% \text { CI: } \\
\quad 58.2-68.5) \\
\text { B. } 48.0(95 \% \text { CI: } \\
\quad 42.5-53.6)\end{array}$ & $\begin{array}{l}\text { A. } 3.7 \\
\text { B. } 1.2\end{array}$ & $\begin{array}{l}\text { A. } 55.7 \\
\text { B. } 47.7\end{array}$ & $\begin{array}{l}\text { Expanded indication: combination } \\
\text { with bevacizumab, paclitaxel, } \\
\text { and carboplatin for first-line treatment } \\
\text { of metastatic NsqNSCLC without } \\
\text { EGFR or ALK mutation (December } \\
\text { 2018) }\end{array}$ \\
\hline & SCLC & $\begin{array}{l}\text { Horn et al. } \\
\text { (2018) [79] } \\
\text { IMpower133 }\end{array}$ & III & $\begin{array}{l}\text { Extensive stage } \\
\text { SCLC without } \\
\text { prior treatment }\end{array}$ & $\begin{array}{l}\text { A. Atezolizumab } \\
\text { 1200mg + carboplatin } \\
5 \mathrm{mg} / \mathrm{mL} / \mathrm{min}+ \\
\text { etoposide } 100 \mathrm{mg} / \mathrm{m}^{2} \\
\text { 4 cycles, then } \\
\text { maintenance } \\
\text { atezolizumab } \\
\text { 12000m Q3wks. } \\
\text { B. Placebo + } \\
\text { carboplatin + } \\
\text { etoposide at doses } \\
\text { above x4 cycles, then } \\
\text { placebo afterward }\end{array}$ & $\begin{array}{c}403 \\
\mathbf{A}=201 \\
\mathbf{B}=202\end{array}$ & $\begin{array}{c}\text { A. } 12.3 \text { (95\% CI: } \\
10.8-15.9) \\
\text { B. } 10.3(95 \% \text { CI: } \\
9.3-11.3) \\
(\mathrm{HR}=0.70 \\
p=0.0069)\end{array}$ & $\begin{array}{c}\text { A. } 5.2(95 \% \text { CI: } \\
\quad 4.4-5.6) \\
\text { B. } 4.3(95 \% \text { CI: } \\
4.2-5.4) \\
(\mathrm{HR}=0.77 \\
p=0.0170)\end{array}$ & $\begin{array}{l}\text { A. } 60.2(95 \% \mathrm{CI}: \\
53.1-67.0) \\
\text { B. } 64.4(95 \% \mathrm{CI}: \\
57.3-71.0)\end{array}$ & $\begin{array}{l}\text { A. } 2.5 \\
\text { B. } 1.0\end{array}$ & $\begin{array}{l}\text { A. } 56.6 \\
\text { B. } 56.1\end{array}$ & $\begin{array}{l}\text { Combination with carboplatin and } \\
\text { etoposide for first-line treatment in } \\
\text { patients with extensive stage SCLC } \\
\text { (March 2019) }\end{array}$ \\
\hline \multirow[t]{3}{*}{ Avelumab } & Bladder cancer & $\begin{array}{l}\text { Apolo et al. } \\
\text { (2017) [80] } \\
\text { JAVELIN }\end{array}$ & $\mathrm{Ib}$ & $\begin{array}{l}\text { Metastatic } \\
\text { urothelial } \\
\text { carcinoma } \\
\text { refractory to at } \\
\text { least 1 previous } \\
\text { treatment }\end{array}$ & $\begin{array}{c}\text { Avelumab 10mg } / \mathrm{kg} \\
\text { Q2wks. }\end{array}$ & 44 & $\begin{array}{l}13.7 \text { ( } 95 \% \mathrm{CI}: \\
\quad 8.5-\mathrm{NE})\end{array}$ & $\begin{array}{l}11.6(95 \% \text { CI: } \\
6.1-17.4)\end{array}$ & $\begin{array}{l}18.2(95 \% \mathrm{CI}: \\
8.2-32.7)\end{array}$ & 11.4 & 6.8 & $\begin{array}{l}\text { Locally advanced or metastatic } \\
\text { urothelial carcinoma with disease } \\
\text { progression during or following } \\
\text { platinum chemotherapy or within } 12 \\
\text { months of neoadjuvant or adjuvant } \\
\text { chemotherapy (May 2017) }\end{array}$ \\
\hline & MCC & $\begin{array}{l}\text { Kaufman et al. } \\
\text { (2016) [81] } \\
\text { JAVELIN } \\
\text { Merkel } \\
200\end{array}$ & II & $\begin{array}{l}\text { Stage IV, } \\
\text { therapy } \\
\text { refractory } \\
\text { disease }\end{array}$ & $\begin{array}{c}\text { Avelumab } 10 \mathrm{mg} / \mathrm{kg} \\
\text { Q2wks. }\end{array}$ & 88 & $\begin{array}{l}11.3(95 \% \text { CI: } \\
7.5-14.0)\end{array}$ & $\begin{array}{l}2.7(95 \% \text { CI: } \\
\quad 1.4-6.9)\end{array}$ & $\begin{array}{l}31.8(95 \% \text { CI: } \\
21.9-43.1)\end{array}$ & 9 & 5 & $\begin{array}{l}\text { Treatment of patients } 12 \text { years and } \\
\text { older with metastatic MCC (March } \\
\text { 2017) }\end{array}$ \\
\hline & NSCLC & $\begin{array}{l}\text { Barlesietal. } \\
\text { (2018) [169] } \\
\text { JAVELIN } \\
\text { Lung 200 }\end{array}$ & III & $\begin{array}{l}\text { Stage IIIb/IV or } \\
\text { recurrent } \\
\text { disease with } \\
\text { progression } \\
\text { after } \\
\text { treatment with a } \\
\text { platinum-containing } \\
\text { doublet }\end{array}$ & $\begin{array}{l}\text { A. Avelumab } \\
10 \mathrm{~m} / \mathrm{kg} \text { Q2wks } \\
\text { B. Docetaxel } 75 \\
\mathrm{mg} / \mathrm{m}^{2} \mathrm{Q} 3 \mathrm{wks} \\
\end{array}$ & $\begin{array}{c}792 \\
\mathbf{A}=396 \\
\mathbf{B}=396\end{array}$ & $\begin{array}{c}\text { A. } 10.5(95 \% \text { CI: } \\
\quad 9.2-12.9) \\
\text { B. } 9.9(95 \% \mathrm{CI} \\
\quad 8.1-11.8) \\
\text { HR }=0.90 \\
p=0.12\end{array}$ & $\begin{array}{c}\text { A. } 2.8(95 \% \mathrm{CI}: \\
2.7-3.5) \\
\text { B. } 4.2(95 \% \mathrm{CI}: \\
3.3-5.2) \\
\mathrm{HR}=1.16 \\
p=0.95\end{array}$ & $\begin{array}{l}\text { A. } 15 \\
\text { B. } 11 \\
\text { Odds ratio }=1.40 \\
p=0.055\end{array}$ & $\begin{array}{l}\text { A. } 1 \\
\text { B. } 1\end{array}$ & $\begin{array}{l}\text { A. } 10 \\
\text { B. } 49\end{array}$ & \\
\hline
\end{tabular}


Table A5. Cont.

\begin{tabular}{|c|c|c|c|c|c|c|c|c|c|c|c|c|}
\hline Therapy & Cancer & Study & Phase & $\begin{array}{l}\text { Stage of } \\
\text { Disease }\end{array}$ & Treatment Regimen & $\mathbf{N}$ & $\begin{array}{c}\text { Median OS } \\
\text { (months) }\end{array}$ & $\begin{array}{c}\text { Median PFS } \\
\text { (months) }\end{array}$ & ORR (\%) & CRR (\%) & $\begin{array}{c}\text { Grade } \\
\text { III/IV } \\
\text { AEs (\%) }\end{array}$ & $\begin{array}{l}\text { Resultant FDA-Approved } \\
\text { Indication }\end{array}$ \\
\hline & RCC & $\begin{array}{l}\text { Motzer et al. } \\
(2019)[82] \\
\text { JAVELIN } \\
\text { Renal } 101\end{array}$ & III & $\begin{array}{l}\text { Previously } \\
\text { untreated } \\
\text { disease }\end{array}$ & $\begin{array}{c}\text { A. Avelumab } \\
\text { 10mg/kg Q2wks. + } \\
\text { axitinib 5mg BID } \\
\text { B. Sunitinib 50mg } \\
\text { QD }\end{array}$ & $\begin{array}{c}886 \\
\mathbf{A}=442 \\
\mathbf{B}=444\end{array}$ & $\begin{array}{l}\text { NR for both } \\
\text { groups } \\
(\mathrm{HR}=0.78 \\
p=0.14)\end{array}$ & $\begin{array}{c}\text { A. } 13.8(95 \% \mathrm{CI}: \\
11.1-\mathrm{NE}) \\
\text { B. } 7.2(95 \% \mathrm{CI} \\
5.7-9.7) \\
(\mathrm{HR}=0.61 \\
p<0.001)\end{array}$ & $\begin{array}{c}\text { Overall: } \\
\text { A. } 51.4(95 \%) \text { CI: } \\
46.6-56.1) \\
\text { B. } 25.7 \text { (95\% CI: } \\
21.7-30.0) \\
\text { PD-L1+: } \\
\text { A. } 55.2(95 \% \text { CI: } \\
49.0-61.2) \\
\text { B. } 25.5(95 \% \text { CI: } \\
20.6-30.9)\end{array}$ & $\begin{array}{l}\frac{\text { Overall: }}{\text { A. } 3.4} \\
\text { B. } 1.8 \\
\text { PD-L1+: } \\
\text { A. } 4.4 \\
\text { B. } 2.1\end{array}$ & $\begin{array}{l}\text { A. } 71.2 \\
\text { B. } 71.5\end{array}$ & $\begin{array}{l}\text { Combination with axitinib for } \\
\text { first-line treatment for advanced RCC } \\
\text { (May 2019) }\end{array}$ \\
\hline \multirow[t]{4}{*}{ Durvalumab } & Bladder cancer & $\begin{array}{l}\text { Massard et al. } \\
\text { (2016) [83] }\end{array}$ & $I / I I$ & $\begin{array}{l}\text { Metastatic } \\
\text { urothelial } \\
\text { cancer }\end{array}$ & $\begin{array}{c}\text { Durvalumab } \\
\text { 10mg/kg Q2wks. }\end{array}$ & 61 & - & - & $\begin{array}{c}\text { Overall: } \\
\text { 31\% (95\% CI: } \\
\text { 17.6-47.4) } \\
\text { PD-L1 positive: } \\
46.4 \%(95 \% \text { CI: } \\
27.5-66.1) \\
\text { PD-L1 negative: } \\
0 \% \text { (95\% CI: } 0.0-23.2)\end{array}$ & - & 4.9 & - \\
\hline & & $\begin{array}{l}\text { Powles et al. } \\
\text { (2017) [170] } \\
\text { (updated } \\
\text { results of } \\
\text { previous } \\
\text { study) }\end{array}$ & $I / I I$ & $\begin{array}{l}\text { Metastatic } \\
\text { Urothelial } \\
\text { Cancer }\end{array}$ & $\begin{array}{c}\text { Durvalumab } \\
\text { 10mg/kg Q2wks. }\end{array}$ & 191 & $\begin{array}{c}\text { Overall: } 18.2 \\
\text { (95\% CI: } \\
8.1-N E) \\
\text { PD-L1 high: } \\
20.0 \text { (95\% CI: } \\
11.6-\mathrm{NE}) \\
\text { PD-L1 low/-: } \\
\text { 8.1 (95\% CI: } \\
3.1-\mathrm{NE})\end{array}$ & $\begin{array}{c}\text { Overall: } 1.5 \\
\text { (95\% CI: } 1.4-1.9) \\
\text { PD-L1 high: } 2.1 \\
\text { (95\% CI: } 1.4-2.8) \\
\text { PD-L1 low/-: } \\
1.4(95 \% \text { CI: } \\
\text { 1.3-1.5) }\end{array}$ & $\begin{array}{c}\text { Overall: } 17.8(95 \% \text { CI: } \\
12.7-24.0 \% \\
\text { PD-L1 high: } 27.6 \\
\text { (95\% CI: } 19-37.5) \\
\text { PD-L1 low/:- } 5.1 \\
\text { (95\% CI: 1.4-12.5) }\end{array}$ & $\begin{array}{l}\frac{\text { Overall: }}{3.7} \\
\frac{\text { PD-L1 high: }}{4.1} \\
\frac{\text { PD-L1 low/-: }}{2.5}\end{array}$ & 6.8 & $\begin{array}{l}\text { Locally advanced or metastatic } \\
\text { urothelial carcinoma with disease } \\
\text { progression during or following } \\
\text { platinum chemotherapy or } \\
\text { progression within } 12 \text { months of } \\
\text { neoadjuvant or adjuvant treatment } \\
\text { with platinum therapy (May 2017) }\end{array}$ \\
\hline & Mesothelioma & $\begin{array}{l}\text { Nowak et al. } \\
\text { (2018) [171] } \\
\text { DREAM }\end{array}$ & II & $\begin{array}{l}\text { Radiation naive } \\
\text { malignant } \\
\text { pleural } \\
\text { mesothelioma }\end{array}$ & $\begin{array}{c}\text { Durvalumab } 1125 \mathrm{mg} \\
+ \text { cisplatin } 75 \mathrm{mg} / \mathrm{m}^{2} \\
\text { +pemetrexed } 500 \\
\mathrm{mg} / \mathrm{m}^{2} \text { 3-weekly x6 } \\
\text { followed by } \\
\text { durvalumab } 1125 \mathrm{mg} \\
\text { 3-weekly }\end{array}$ & 54 & - & - & 61 & - & 57 & - \\
\hline & NSCLC & $\begin{array}{l}\text { Antonia et al. } \\
\text { (2017) [84] } \\
\text { PACIFIC }\end{array}$ & Phase III & $\begin{array}{c}\text { Stage III } \\
\text { NSCLC who } \\
\text { did not have } \\
\text { progression } \\
\text { after } 2 \text { or more } \\
\text { cycles of } \\
\text { platinum-based } \\
\text { chemotherapy }\end{array}$ & $\begin{array}{l}\text { A. Durvalumab } \\
\text { 10mg/kg Q2wks. } \\
\text { B. Placebo }\end{array}$ & $\begin{aligned} 713 \\
\mathbf{A}=473 \\
\mathbf{B}=236\end{aligned}$ & $\begin{array}{l}\text { Not done at } \\
\text { time of study }\end{array}$ & $\begin{array}{c}\text { A. } 16.8(95 \% \text { CI: } \\
13.0-18.1) \\
\text { B. } 5.6(95 \% \text { CI: } \\
4.6-7.8) \\
(\mathrm{HR}=0.52 \\
p<0.001)\end{array}$ & $\begin{array}{l}\text { A. } 28.4(95 \% \mathrm{CI}: \\
24.3-32.9) \\
\text { B. } 16(95 \% \mathrm{CI}: \\
11.3-21.6) \\
(p<0.001)\end{array}$ & $\begin{array}{l}\text { A. } 1.4 \\
\text { B. } 0.5\end{array}$ & $\begin{array}{l}\text { A. } 29.9 \\
\text { B. } 26.1\end{array}$ & $\begin{array}{l}\text { Consolidation therapy for } \\
\text { unresectable stage III NSCLC that has } \\
\text { not progressed following concurrent } \\
\text { platinum-based chemotherapy and } \\
\text { radiation therapy }\end{array}$ \\
\hline
\end{tabular}

ALK = anaplastic lymphoma kinase, $\mathrm{CI}=$ confidence interval, $\mathrm{EGFR}=$ epidermal growth factor receptor, $\mathrm{HR}=$ hazard ratio, $\mathrm{MCC}=\mathrm{Merkel}$ cell carcinoma, $\mathrm{NE}=$ not estimable, $\mathrm{NR}=$ not reached, NSCLC = non-small cell lung cancer, NsqNSCLC = non-squamous non-small cell lung cancer, $\mathrm{RCC}=$ renal cell carcinoma, SCLC $=$ small cell lung cancer, TNBC = triple-negative breast cancer. 
Table A6. Ongoing trials involving anti-LAG-3 and anti-TIM-3 therapies.

\begin{tabular}{|c|c|c|c|c|c|c|c|c|}
\hline $\begin{array}{l}\text { ClinicalTrial.gov } \\
\text { Identifier }\end{array}$ & Year Opened & $\operatorname{Drug}(\mathbf{s})$ & Class & Phase & $\begin{array}{c}\text { Estimated } \\
\text { Enrollment }\end{array}$ & Disease & Arms/Interventions & $\begin{array}{l}\text { Anticipated } \\
\text { Completion }\end{array}$ \\
\hline NCT01968109 & 2013 & $\begin{array}{l}\text { 1. Relatlimab } \\
\text { 2. Nivolumab }\end{array}$ & $\begin{array}{l}\text { 1. Anti-LAG-3 } \\
\text { 2. Anti-PD-1 }\end{array}$ & $\mathrm{I} / \mathrm{II} \mathrm{a}$ & 2000 & Advanced solid tumors & $\begin{array}{c}\text { Relatlimab dose } \\
\text { escalation and cohort } \\
\text { expansion study alone or } \\
\text { in combination with } \\
\text { nivolumab }\end{array}$ & 2023 \\
\hline NCT02060188 & 2014 & $\begin{array}{l}\text { 1. Nivolumab } \\
\text { 2. Ipilimumab } \\
\text { 3. Cobimetinib } \\
\text { 4. Daratumumab } \\
\text { 5. Relatlimab }\end{array}$ & $\begin{array}{l}\text { 1. Anti-PD-1 } \\
\text { 2. Anti-CTLA-4 } \\
\text { 3. MEK } \\
\text { inhibitor } \\
\text { 4. Anti-CD38 } \\
\text { 5. Anti-LAG3 }\end{array}$ & II & 340 & $\begin{array}{l}\text { Recurrent and metastatic } \\
\text { MSI-H and non-MSI-H } \\
\text { colon cancer }\end{array}$ & $\begin{array}{l}\text { Nivolumab alone or in } \\
\text { combination with } \\
\text { ipilimumab, cobimetinib, } \\
\text { daratumumab, or } \\
\text { relatlimab }\end{array}$ & 2020 \\
\hline NCT02061761 & 2014 & $\begin{array}{l}\text { 1. Relatlimab } \\
\text { 2. Nivolumab }\end{array}$ & $\begin{array}{l}\text { 1. Anti-LAG-3 } \\
\text { 2. Anti-PD-1 }\end{array}$ & I/IIa & 132 & $\begin{array}{l}\text { Relapsed or refractory } \\
\text { B-cell malignancies }\end{array}$ & $\begin{array}{c}\text { Relatlimab alone or in } \\
\text { combination with } \\
\text { nivolumab }\end{array}$ & 2020 \\
\hline NCT02488759 & 2015 & $\begin{array}{l}\text { 1. Nivolumab } \\
\text { 2. Ipilimumab } \\
\text { 3. Relatlimab } \\
\text { 4. Daratumumab }\end{array}$ & $\begin{array}{l}\text { 1. Anti-PD-1 } \\
\text { 2. Anti-CTLA-4 } \\
\text { 3. Anti-LAG-3 } \\
\text { 4. Anti-CD38 }\end{array}$ & $\mathrm{I} / \mathrm{II}$ & 600 & $\begin{array}{l}\text { Virus-positive and } \\
\text { virus-negative solid } \\
\text { tumors }\end{array}$ & $\begin{array}{l}\text { Nivolumab alone or in } \\
\text { combination with } \\
\text { ipilimumab, relatlimab, } \\
\text { or daratumumab }\end{array}$ & 2019 \\
\hline NCT02608268 & 2015 & $\begin{array}{l}\text { 1. MBG453 } \\
\text { 2. Spartalizumab }\end{array}$ & $\begin{array}{l}\text { 1. Anti-TIM-3 } \\
\text { 2. Anti-PD-1 }\end{array}$ & I & 250 & Advanced solid tumors & $\begin{array}{l}\text { MPG453 alone or in } \\
\text { combination with } \\
\text { spartalizumab }\end{array}$ & 2019 \\
\hline NCT02658981 & 2016 & $\begin{array}{l}\text { 1. Relatlimab } \\
\text { 2. Urelumab } \\
\text { 3. Nivolumab }\end{array}$ & $\begin{array}{l}\text { 1. Anti-LAG-3 } \\
\text { 2. Anti-CD137 } \\
\text { 3. Anti-PD-1 }\end{array}$ & I & 100 & Recurrent GBM & $\begin{array}{l}\text { Relatlimab or urelumab } \\
\text { alone or in combination } \\
\text { with nivolumab }\end{array}$ & 2020 \\
\hline NCT02750514 & 2016 & $\begin{array}{l}\text { 1. Nivolumab } \\
\text { 2. Ipilimumab } \\
\text { 3. Relatlimab } \\
\text { 4. Dasatinib } \\
\text { 5. BMS-986205 }\end{array}$ & $\begin{array}{l}\text { 1. Anti-PD-1 } \\
\text { 2. Anti-CTLA-4 } \\
\text { 3. Anti-LAG-3 } \\
\text { 4. TK inhibitor } \\
\text { 5. Anti-IDO1 }\end{array}$ & II & 504 & Advanced NSCLC & $\begin{array}{c}\text { Nivolumab alone or } \\
\text { nivolumab + ipilimumab } \\
\text { or nivolumab }+ \\
\text { relatlimab or nivolumab } \\
+ \text { dasatinib or nivolumab } \\
+ \text { BMS-986205 }\end{array}$ & 2021 \\
\hline NCT02935634 & 2016 & $\begin{array}{l}\text { 1. Nivolumab } \\
\text { 2. Ipilimumab } \\
\text { 3. Relatlimab } \\
\text { 4. BMS-986205 }\end{array}$ & $\begin{array}{l}\text { 1. Anti-PD-1 } \\
\text { 2. Anti-CTLA-4 } \\
\text { 3. Anti-LAG-3 } \\
\text { 4. Anti-IDO1 }\end{array}$ & II & 300 & Advanced gastric cancer & $\begin{array}{l}\text { Nivolumab + ipilimumab } \\
\text { or nivolumab }+ \\
\text { relatlimab or nivolumab } \\
+ \text { BMS-986205 }\end{array}$ & 2021 \\
\hline NCT02996110 & 2016 & $\begin{array}{l}\text { 1. Nivolumab } \\
\text { 2. Ipilimumab } \\
\text { 3. Relatlimab } \\
\text { 4. BMS-986205 } \\
\text { 5. BMS-813160 }\end{array}$ & $\begin{array}{l}\text { 1. Anti-PD-1 } \\
\text { 2. Anti-CTLA-4 } \\
\text { 3. Anti-LAG-3 } \\
\text { 4. Anti-IDO1 } \\
\text { 5. } \\
\text { Anti-CCR2/CCR5 }\end{array}$ & II & 200 & Advanced RCC & $\begin{array}{c}\text { Nivolumab + ipilimumab } \\
\text { or nivolumab + } \\
\text { relatlimab or nivolumab } \\
+ \text { BMS-986205 or } \\
\text { nivolumab + BMS-813160 }\end{array}$ & 2022 \\
\hline
\end{tabular}


Table A6. Cont.

\begin{tabular}{|c|c|c|c|c|c|c|c|c|}
\hline $\begin{array}{l}\text { ClinicalTrial.gov } \\
\text { Identifier }\end{array}$ & Year Opened & Drug(s) & Class & Phase & $\begin{array}{c}\text { Estimated } \\
\text { Enrollment }\end{array}$ & Disease & Arms/Interventions & $\begin{array}{l}\text { Anticipated } \\
\text { Completion }\end{array}$ \\
\hline NCT03311412 & 2017 & $\begin{array}{l}\text { 1. Sym021 } \\
\text { 2. Sym022 } \\
\text { 3. Sym023 }\end{array}$ & $\begin{array}{l}\text { 1. Anti-PD-1 } \\
\text { 2. Anti-LAG-3 } \\
\text { 3. Anti-TIM-3 }\end{array}$ & I & 102 & $\begin{array}{l}\text { Advanced solid tumors } \\
\text { or lymphomas }\end{array}$ & $\begin{array}{l}\text { Sym021 alone or in } \\
\text { combination with either } \\
\text { Sym022 or Sym023 }\end{array}$ & 2020 \\
\hline NCT03219268 & 2017 & $\begin{array}{l}\text { 1. MGD013 } \\
\text { 2. Margetuximab }\end{array}$ & $\begin{array}{l}\text { 1. Dual } \\
\text { anti-PD-1/LAG-3 } \\
\text { 2. Anti-HER2 }\end{array}$ & I & 255 & $\begin{array}{l}\text { (A) Unresectable or } \\
\text { metastatic solid } \\
\text { neoplasms } \\
\text { (B) HER2-positive breast } \\
\text { cancer }\end{array}$ & $\begin{array}{l}\text { (A) MGD013 dose } \\
\text { escalation study } \\
\text { (B) MGD013 in } \\
\text { combination with } \\
\text { margetuximab }\end{array}$ & 2022 \\
\hline NCT03470922 & 2018 & $\begin{array}{l}\text { 1. Nivolumab } \\
\text { 2. Relatlimab }\end{array}$ & $\begin{array}{l}\text { 1. Anti-PD-1 } \\
\text { 2. Anti-LAG-3 }\end{array}$ & $\mathrm{II} / \mathrm{III}$ & 700 & $\begin{array}{l}\text { Previously untreated or } \\
\text { unresectable melanoma }\end{array}$ & $\begin{array}{l}\text { Nivolumab alone or in } \\
\text { combination with } \\
\text { relatlimab }\end{array}$ & 2022 \\
\hline NCT03440437 & 2018 & FS118 & $\begin{array}{c}\text { Dual } \\
\text { anti-PD-L1/LAG-3 }\end{array}$ & I & 51 & $\begin{array}{l}\text { Advanced malignancies } \\
\text { that progressed on or } \\
\text { after PD-1/PD-L1 } \\
\text { containing therapy }\end{array}$ & $\begin{array}{l}\text { FS118 dose escalation and } \\
\text { cohort expansion study }\end{array}$ & 2020 \\
\hline NCT03623854 & 2018 & $\begin{array}{l}\text { 1. Relatlimab } \\
\text { 2. Nivolumab }\end{array}$ & $\begin{array}{l}\text { 1. Anti-LAG-3 } \\
\text { 2. Anti-PD-1 }\end{array}$ & II & 20 & Advanced chordoma & $\begin{array}{l}\text { Relatlimab in } \\
\text { combination with } \\
\text { nivolumab }\end{array}$ & 2022 \\
\hline NCT03610711 & 2018 & $\begin{array}{l}\text { 1. Nivolumab } \\
\text { 2. Relatlimab }\end{array}$ & $\begin{array}{l}\text { 1. Anti-PD-1 } \\
\text { 2. Anti-LAG-3 }\end{array}$ & $\mathrm{I} / \mathrm{II}$ & 30 & $\begin{array}{c}\text { Advanced } \\
\text { esophagogastric cancer } \\
\text { following targeted } \\
\text { systemic radiation }\end{array}$ & $\begin{array}{l}\text { Nivolumab alone or in } \\
\text { combination with } \\
\text { relatlimab }\end{array}$ & 2024 \\
\hline NCT03743766 & 2018 & $\begin{array}{l}\text { 1. Nivolumab } \\
\text { 2. Relatlimab }\end{array}$ & $\begin{array}{l}\text { 1. Anti-PD-1 } \\
\text { 2. Anti-LAG-3 }\end{array}$ & II & 42 & $\begin{array}{l}\text { Metastatic melanoma } \\
\text { naïve to prior } \\
\text { immunotherapy }\end{array}$ & $\begin{array}{c}\text { Nivolumab alone or } \\
\text { relatlimab alone or } \\
\text { combination nivolumab + } \\
\text { relatlimab }\end{array}$ & 2022 \\
\hline NCT03459222 & 2018 & $\begin{array}{l}\text { 1. Relatlimab } \\
\text { 2. Nivolumab } \\
\text { 3. BMS-986205 } \\
\text { 4. Ipilimumab }\end{array}$ & $\begin{array}{l}\text { 1. Anti-LAG-3 } \\
\text { 2. Anti-PD-1 } \\
\text { 3. Anti-IDO1 } \\
\text { 4. Anti-CTLA-4 }\end{array}$ & $\mathrm{I} / \mathrm{II}$ & 230 & Advanced solid tumors & $\begin{array}{c}\text { Relatlimab in } \\
\text { combination with } \\
\text { nivolumab + BMS-986205 } \\
\text { or relatlimab in } \\
\text { combination with } \\
\text { nivolumab + ipilimumab }\end{array}$ & 2022 \\
\hline NCT03744468 & 2018 & $\begin{array}{l}\text { 1. BGB-A425 } \\
\text { 2. Tislelizumab }\end{array}$ & $\begin{array}{l}\text { 1. Anti-TIM-3 } \\
\text { 2. Anti-PD-1 }\end{array}$ & I/II & 162 & Advanced solid tumors & $\begin{array}{l}\text { BGB-A425 in } \\
\text { combination with } \\
\text { tislelizumab }\end{array}$ & 2021 \\
\hline NCT03680508 & 2018 & $\begin{array}{l}\text { 1. TSR-022 } \\
\text { 2. TSR-042 }\end{array}$ & $\begin{array}{l}\text { 1. Anti-TIM-3 } \\
\text { 2. Anti-PD-1 }\end{array}$ & II & 42 & Advanced HCC & $\begin{array}{l}\text { TSR- } 022 \text { in combination } \\
\text { with TSR-042 }\end{array}$ & 2022 \\
\hline NCT03961971 & 2019 & $\begin{array}{l}\text { 1. MBG453 } \\
\text { 2. Spartalizumab }\end{array}$ & $\begin{array}{l}\text { 1. Anti-TIM-3 } \\
\text { 2. Anti-PD-1 }\end{array}$ & I & 15 & Recurrent GBM & $\begin{array}{l}\text { MBG453 in combination } \\
\text { with spartalizumab and } \\
\text { stereotactic radiosurgery }\end{array}$ & 2023 \\
\hline
\end{tabular}

GBM = glioblastoma multiforme, HCC = hepatocellular carcinoma, MSI-H = microsatellite instability high, NSCLC = non-small cell lung cancer, RCC = renal cell carcinoma,

TK = tyrosine kinase. 
Table A7. Ongoing trials involving CD40 agonists or OX40 agonists.

\begin{tabular}{|c|c|c|c|c|c|c|c|c|}
\hline $\begin{array}{l}\text { ClinicalTrial.Gov } \\
\text { Identifier }\end{array}$ & Year Opened & Drug(s) & Class & Phase & $\begin{array}{c}\text { Estimated } \\
\text { Enrollment }\end{array}$ & Disease & Arms/Interventions & $\begin{array}{l}\text { Anticipated } \\
\text { Completion }\end{array}$ \\
\hline NCT02304393 & 2014 & $\begin{array}{l}\text { 1. Atezolizumab } \\
\text { 2. Selicrelumab }\end{array}$ & $\begin{array}{l}\text { 1. Anti-PD-L1 } \\
\text { 2. CD40 agonist }\end{array}$ & I & 142 & $\begin{array}{l}\text { Advanced solid } \\
\text { malignancies }\end{array}$ & $\begin{array}{l}\text { Dose escalation of selicrelumab in } \\
\text { combination with atezolizumab } \\
\text { followed by dose expansion of dose } \\
\text { expansion of selicrelumab in } \\
\text { combination with atezolizumab }\end{array}$ & 2019 \\
\hline NCT02665416 & 2016 & $\begin{array}{l}\text { 1. Selicrelumab } \\
\text { 2. Vanucizumab } \\
\text { 3. Bevacizumab }\end{array}$ & $\begin{array}{l}\text { 1. CD40 agonist } \\
\text { 2. Anti-VEGF-A } \\
\text { and Anti-Ang-2 } \\
\text { 3. Anti-VEGF-A }\end{array}$ & I/II & 170 & $\begin{array}{l}\text { Advanced or metastatic } \\
\text { solid tumors }\end{array}$ & $\begin{array}{l}\text { Dose escalation of selicrelumab in } \\
\text { combination with vanucizumab } \\
\text { followed by dose expansion of } \\
\text { selicrelumab in combination with } \\
\text { bevacizumab }\end{array}$ & 2020 \\
\hline NCT03092856 & 2017 & $\begin{array}{l}\text { 1. Axitinib } \\
\text { 2. PF-04518600 }\end{array}$ & $\begin{array}{l}\text { 1. TK Inhibitor } \\
\text { 2. OX40 agonist }\end{array}$ & II & 104 & $\begin{array}{l}\text { Metastatic or recurrent } \\
\text { RCC }\end{array}$ & $\begin{array}{l}\text { Axitinib alone or in combination with } \\
\text { PF- } 04518600\end{array}$ & 2021 \\
\hline NCT02706353 & 2017 & $\begin{array}{l}\text { 1. APX005M } \\
\text { 2. Pembrolizumab }\end{array}$ & $\begin{array}{l}\text { 1. CD40 agonist } \\
\text { 2. Anti-PD-1 }\end{array}$ & I/II & 41 & Metastatic melanoma & $\begin{array}{c}\text { Dose escalation of APX005M in } \\
\text { combination with pembrolizumab } \\
\text { followed by dose expansion of } \\
\text { APX005M in combination with } \\
\text { pembrolizumab }\end{array}$ & 2020 \\
\hline NCT03217747 & 2017 & $\begin{array}{l}\text { 1. Avelumab2. } \\
\text { Utomilumab } \\
\text { 3. PF-04518600 }\end{array}$ & $\begin{array}{l}\text { 1. Anti-PD-L12. } \\
\text { Anti-CD137 } \\
\text { 3. OX40 agonist }\end{array}$ & I/II & 184 & $\begin{array}{l}\text { Advanced solid } \\
\text { malignancies }\end{array}$ & $\begin{array}{l}\text { Avelumab }+ \text { utomilumab or avelumab }+ \\
\text { PF-04518600 or avelumab + utomilumab } \\
\quad+\text { PF-04518600 or avelumab + RT }\end{array}$ & 2023 \\
\hline NCT03410901 & 2018 & $\begin{array}{l}\text { 1. SD-101 } \\
\text { 2. BMS } 986178\end{array}$ & $\begin{array}{l}\text { 1. TLR9 Agonist } \\
\text { 2. OX40 agonist }\end{array}$ & I & 15 & Advanced lymphomas & Combination SD-101 + BMS 986178 + RT & 2020 \\
\hline NCT03389802 & 2018 & 1. APX005M & 1. CD40 agonist & $\mathrm{I}$ & 45 & Pediatric CNS tumors & Dose escalation of APX005M alone & 2022 \\
\hline NCT03336606 & 2018 & 1. MEDI0562 & 1. OX40 agonist & I & 35 & HNSCC, melanoma & $\begin{array}{l}\text { MEDI0562 at varying dose schedules } \\
\text { prior to surgical resection }\end{array}$ & 2024 \\
\hline NCT03892525 & 2019 & $\begin{array}{l}\text { 1. Selicrelumab } \\
\text { 2. Atezolizumab }\end{array}$ & $\begin{array}{l}\text { 1. CD40 agonist } \\
\text { 2. Anti-PD-L1 }\end{array}$ & I & 44 & $\begin{array}{l}\text { Recurrent or refractory } \\
\text { NHL }\end{array}$ & $\begin{array}{l}\text { Intratumoral selicrelumab in } \\
\text { combination with atezolizumab }\end{array}$ & 2023 \\
\hline NCT03719430 & 2019 & $\begin{array}{l}\text { 1. Doxorubicin } \\
\text { 2. APX005M }\end{array}$ & $\begin{array}{l}\text { 1. Anthracycline } \\
\text { 2. CD40 agonist }\end{array}$ & II & 27 & $\begin{array}{l}\text { Advanced soft tissue } \\
\text { sarcoma }\end{array}$ & Combination doxorubicin $+\mathrm{APX} 005 \mathrm{M}$ & 2023 \\
\hline
\end{tabular}

CNS = central nervous system, HNSCC = head and neck squamous cell carcinoma, NHL = non-Hodgkin lymphoma, RCC = renal cell carcinoma, RT = radiation therapy, TK = tyrosine kinase. 


\section{References}

1. Brunet, J.-F.; Denizot, F.; Luciani, M.-F.; Roux-Dosseto, M.; Suzan, M.; Mattei, M.-G.; Golstein, P. A New Member of the Immunoglobulin Superfamily-CTLA-4. Nature 1987, 328, 267-270. [CrossRef] [PubMed]

2. Leach, D.R.; Krummel, M.F.; Allison, J.P. Enhancement of Antitumor Immunity by CTLA-4 Blockade. Available online: https://link-galegroup-com.ezproxy4.library.arizona.edu/apps/doc/A18175424/AONE?sid= lms (accessed on 11 August 2019).

3. Boomer, J.S.; Green, J.M. An Enigmatic Tail of CD28 Signaling. Cold Spring Harb. Perspect. Biol. 2010, 2, a002436. [CrossRef] [PubMed]

4. He, M.; Chai, Y.; Qi, J.; Zhang, C.W.H.; Tong, Z.; Shi, Y.; Yan, J.; Tan, S.; Gao, G.F. Remarkably Similar CTLA-4 Binding Properties of Therapeutic Ipilimumab and Tremelimumab Antibodies. Oncotarget 2017, 8, 67129-67139. [CrossRef] [PubMed]

5. Hargadon, K.M.; Johnson, C.E.; Williams, C.J. Immune Checkpoint Blockade Therapy for Cancer: An Overview of FDA-Approved Immune Checkpoint Inhibitors. Int. Immunopharmacol. 2018, 62, $29-39$. [CrossRef]

6. Vidarsson, G.; Dekkers, G.; Rispens, T. IgG Subclasses and Allotypes: From Structure to Effector Functions. Front. Immunol. 2014, 5, 520. [CrossRef]

7. Simpson, T.R.; Li, F.; Montalvo-Ortiz, W.; Sepulveda, M.A.; Bergerhoff, K.; Arce, F.; Roddie, C.; Henry, J.Y.; Yagita, H.; Wolchok, J.D.; et al. Fc-Dependent Depletion of Tumor-Infiltrating Regulatory T Cells Co-Defines the Efficacy of Anti-CTLA-4 Therapy against Melanoma. J. Exp. Med. 2013, 210, 1695-1710. [CrossRef]

8. Selby, M.J.; Engelhardt, J.J.; Quigley, M.; Henning, K.A.; Chen, T.; Srinivasan, M.; Korman, A.J. Anti-CTLA-4 Antibodies of IgG2a Isotype Enhance Antitumor Activity through Reduction of Intratumoral Regulatory T Cells. Cancer Immunol. Res. 2013, 1, 32-42. [CrossRef]

9. Du, X.; Tang, F.; Liu, M.; Su, J.; Zhang, Y.; Wu, W.; Devenport, M.; Lazarski, C.A.; Zhang, P.; Wang, X.; et al. A Reappraisal of CTLA-4 Checkpoint Blockade in Cancer Immunotherapy. Cell Res. 2018, 28, 416-432. [CrossRef]

10. Arce Vargas, F.; Furness, A.J.S.; Litchfield, K.; Joshi, K.; Rosenthal, R.; Ghorani, E.; Solomon, I.; Lesko, M.H.; Ruef, N.; Roddie, C.; et al. Fc Effector Function Contributes to the Activity of Human Anti-CTLA-4 Antibodies. Cancer Cell 2018, 33, 649-663.e4. [CrossRef]

11. Kavanagh, B.; O’Brien, S.; Lee, D.; Hou, Y.; Weinberg, V.; Rini, B.; Allison, J.P.; Small, E.J.; Fong, L. CTLA4 Blockade Expands FoxP3+ Regulatory and Activated Effector CD4+ T Cells in a Dose-Dependent Fashion. Blood 2008, 112, 1175-1183. [CrossRef]

12. Retseck, J.; VanderWeele, R.; Lin, H.-M.; Lin, Y.; Butterfield, L.H.; Tarhini, A.A. Phenotypic and Functional Testing of Circulating Regulatory T Cells in Advanced Melanoma Patients Treated with Neoadjuvant Ipilimumab. J. Immunother. Cancer 2016, 4, 38. [CrossRef] [PubMed]

13. Ribas, A.; Comin-Anduix, B.; Economou, J.S.; Donahue, T.R.; De La Rocha, P.; Morris, L.F.; Jalil, J.; Dissette, V.B.; Shintaku, I.P.; Glaspy, J.A.; et al. Intratumoral Immune Cell Infiltrates, FoxP3, and Indoleamine 2,3-Dioxygenase in Patients with Melanoma Undergoing CTLA4 Blockade. Clin. Cancer Res. 2009, 15, 390-399. [CrossRef] [PubMed]

14. Sharma, A.; Subudhi, S.K.; Blando, J.; Scutti, J.; Vence, L.; Wargo, J.A.; Allison, J.P.; Ribas, A.; Sharma, P. Anti-CTLA-4 Immunotherapy Does Not Deplete FOXP3+ Regulatory T Cells (Tregs) in Human Cancers. Clin. Cancer Res. 2018, 25, 1233-1238. [CrossRef] [PubMed]

15. Topalian, S.L.; Hodi, F.S.; Brahmer, J.R.; Gettinger, S.N.; Smith, D.C.; McDermott, D.F.; Powderly, J.D.; Carvajal, R.D.; Sosman, J.A.; Atkins, M.B.; et al. Safety, Activity, and Immune Correlates of Anti-PD-1 Antibody in Cancer. N. Engl. J. Med. 2012, 366, 2443-2454. [CrossRef] [PubMed]

16. Ishida, Y.; Agata, Y.; Shibahara, K.; Honjo, T. Induced Expression of PD-1, a Novel Member of the Immunoglobulin Gene Superfamily, upon Programmed Cell Death. EMBO J. 1992, 11, 3887-3895. [CrossRef] [PubMed]

17. Agata, Y.; Kawasaki, A.; Nishimura, H.; Ishida, Y.; Tsubata, T.; Yagita, H.; Honjo, T. Expression of the PD-1 Antigen on the Surface of Stimulated Mouse T and B Lymphocytes. Int. Immunol. 1996, 8, 765-772. [CrossRef] [PubMed]

18. Postow, M.A.; Callahan, M.K.; Wolchok, J.D. Immune Checkpoint Blockade in Cancer Therapy. J. Clin. Oncol. 2015, 33, 1974-1982. [CrossRef] 
19. Seidel, J.A.; Otsuka, A.; Kabashima, K. Anti-PD-1 and Anti-CTLA-4 Therapies in Cancer: Mechanisms of Action, Efficacy, and Limitations. Front. Oncol. 2018, 8, 86. [CrossRef]

20. Zhang, N.; Tu, J.; Wang, X.; Chu, Q. Programmed Cell Death-1/Programmed Cell Death Ligand-1 Checkpoint Inhibitors: Differences in Mechanism of Action. Immunotherapy 2019, 11, 429-441. [CrossRef]

21. Lee, J.Y.; Lee, H.T.; Shin, W.; Chae, J.; Choi, J.; Kim, S.H.; Lim, H.; Won Heo, T.; Park, K.Y.; Lee, Y.J.; et al. Structural Basis of Checkpoint Blockade by Monoclonal Antibodies in Cancer Immunotherapy. Nat. Commun. 2016, 7. [CrossRef]

22. Flies, D.B.; Chen, L. The New B7s: Playing a Pivotal Role in Tumor Immunity. J. Immunother. 2007, 30, 251-260. [CrossRef] [PubMed]

23. Lee, H.T.; Lee, J.Y.; Lim, H.; Lee, S.H.; Moon, Y.J.; Pyo, H.J.; Ryu, S.E.; Shin, W.; Heo, Y.-S. Molecular Mechanism of PD-1/PD-L1 Blockade via Anti-PD-L1 Antibodies Atezolizumab and Durvalumab. Sci. Rep. 2017, 7, 1-12. [CrossRef] [PubMed]

24. Ascierto, P.A.; Bono, P.; Bhatia, S.; Melero, I.; Nyakas, M.S.; Svane, I.-M.; Larkin, J.; Gomez-Roca, C.; Schadendorf, D.; Dummer, R.; et al. LBA18Efficacy of BMS-986016, a Monoclonal Antibody That Targets Lymphocyte Activation Gene-3 (LAG-3), in Combination with Nivolumab in Pts with Melanoma Who Progressed during Prior Anti-PD-1/PD-L1 Therapy (Mel Prior IO) in All-Comer and Biomarker-Enriched Populations. Ann. Oncol. 2017, 28 (Suppl. 5). [CrossRef]

25. Triebel, F.; Jitsukawa, S.; Baixeras, E.; Roman-Roman, S.; Genevee, C.; Viegas-Pequignot, E.; Hercend, T. LAG-3, a Novel Lymphocyte Activation Gene Closely Related to CD4. J. Exp. Med. 1990, 171, 1393-1405. [CrossRef] [PubMed]

26. Huard, B.; Tournier, M.; Hercend, T.; Triebel, F.; Faure, F. Lymphocyte-Activation Gene 3/Major Histocompatibility Complex Class II Interaction Modulates the Antigenic Response of CD4+ T Lymphocytes. Eur. J. Immunol. 1994, 24, 3216-3221. [CrossRef]

27. Long, L.; Zhang, X.; Chen, F.; Pan, Q.; Phiphatwatchara, P.; Zeng, Y.; Chen, H. The Promising Immune Checkpoint LAG-3: From Tumor Microenvironment to Cancer Immunotherapy. Genes Cancer 2018, 9, 176-189. [CrossRef]

28. Andrews, L.P.; Marciscano, A.E.; Drake, C.G.; Vignali, D.A.A. LAG3 (CD223) as a Cancer Immunotherapy Target. Immunol. Rev. 2017, 276, 80-96. [CrossRef]

29. Patel, J.; Bozeman, E.N.; Selvaraj, P. Taming Dendritic Cells with TIM-3: Another Immunosuppressive Strategy Used by Tumors. Immunotherapy 2012, 4, 1795-1798. [CrossRef]

30. He, Y.; Cao, J.; Zhao, C.; Li, X.; Zhou, C.; Hirsch, F.R. TIM-3, a Promising Target for Cancer Immunotherapy. OncoTargets Ther. 2018, 11, 7005-7009. [CrossRef]

31. Vonderheide, R.H. The Immune Revolution: A Case for Priming, Not Checkpoint. Cancer Cell 2018, 33, 563-569. [CrossRef]

32. Dempke, W.C.M.; Fenchel, K.; Uciechowski, P.; Dale, S.P. Second- and Third-Generation Drugs for Immuno-Oncology Treatment-The More the Better? Eur. J. Cancer 2017, 74, 55-72. [CrossRef] [PubMed]

33. van Kooten, C.; Banchereau, J. CD40-CD40 Ligand. J. Leukoc. Biol. 2000, 67, 2-17. [CrossRef] [PubMed]

34. Piechutta, M.; Berghoff, A.S. New Emerging Targets in Cancer Immunotherapy: The Role of Cluster of Differentiation 40 (CD40/TNFR5). ESMO Open 2019, 4 (Suppl. 3), e000510. [CrossRef] [PubMed]

35. Sugamura, K.; Ishii, N.; Weinberg, A.D. Therapeutic Targeting of the Effector T-Cell Co-Stimulatory Molecule OX40. Nat. Rev. Immunol. 2004, 4, 420-431. [CrossRef]

36. Willoughby, J.; Griffiths, J.; Tews, I.; Cragg, M.S. OX40: Structure and Function - What Questions Remain? Mol. Immunol. 2017, 83, 13-22. [CrossRef]

37. So, T.; Song, J.; Sugie, K.; Altman, A.; Croft, M. Signals from OX40 Regulate Nuclear Factor of Activated T Cells C1 and T Cell Helper 2 Lineage Commitment. Proc. Natl. Acad. Sci. USA 2006, 103, 3740-3745. [CrossRef]

38. Hodi, F.S.; O’Day, S.J.; McDermott, D.F.; Weber, R.W.; Sosman, J.A.; Haanen, J.B.; Gonzalez, R.; Robert, C.; Schadendorf, D.; Hassel, J.C.; et al. Improved Survival with Ipilimumab in Patients with Metastatic Melanoma. N. Engl. J. Med. 2010, 363, 711-723. [CrossRef]

39. Motzer, R.J.; Tannir, N.M.; McDermott, D.F.; Arén Frontera, O.; Melichar, B.; Choueiri, T.K.; Plimack, E.R.; Barthélémy, P.; Porta, C.; George, S.; et al. Nivolumab plus Ipilimumab versus Sunitinib in Advanced Renal-Cell Carcinoma. N. Engl. J. Med. 2018, 378, 1277-1290. [CrossRef] 
40. Motzer, R.J.; Rini, B.I.; McDermott, D.F.; Frontera, O.A.; Hammers, H.J.; Carducci, M.A.; Salman, P.; Escudier, B.; Beuselinck, B.; Amin, A.; et al. Nivolumab plus Ipilimumab versus Sunitinib in First-Line Treatment for Advanced Renal Cell Carcinoma: Extended Follow-up of Efficacy and Safety Results from a Randomised, Controlled, Phase 3 Trial. Lancet Oncol. 2019. [CrossRef]

41. Lynch, T.J.; Bondarenko, I.; Luft, A.; Serwatowski, P.; Barlesi, F.; Chacko, R.; Sebastian, M.; Neal, J.; Lu, H.; Cuillerot, J.-M.; et al. Ipilimumab in Combination with Paclitaxel and Carboplatin as First-Line Treatment in Stage IIIB/IV Non-Small-Cell Lung Cancer: Results From a Randomized, Double-Blind, Multicenter Phase II Study. J. Clin. Oncol. 2012, 30, 2046-2054. [CrossRef]

42. Govindan, R.; Szczesna, A.; Ahn, M.-J.; Schneider, C.-P.; Gonzalez Mella, P.F.; Barlesi, F.; Han, B.; Ganea, D.E.; Von Pawel, J.; Vladimirov, V.; et al. Phase III Trial of Ipilimumab Combined with Paclitaxel and Carboplatin in Advanced Squamous Non-Small-Cell Lung Cancer. J. Clin. Oncol. 2017, 35, 3449-3457. [CrossRef] [PubMed]

43. Maio, M.; Scherpereel, A.; Calabrò, L.; Aerts, J.; Cedres Perez, S.; Bearz, A.; Nackaerts, K.; Fennell, D.A.; Kowalski, D.; Tsao, A.S.; et al. Tremelimumab as Second-Line or Third-Line Treatment in Relapsed Malignant Mesothelioma (DETERMINE): A Multicentre, International, Randomised, Double-Blind, Placebo-Controlled Phase 2b Trial. Lancet Oncol. 2017, 18, 1261-1273. [CrossRef]

44. Sharma, P.; Retz, M.; Siefker-Radtke, A.; Baron, A.; Necchi, A.; Bedke, J.; Plimack, E.R.; Vaena, D.; Grimm, M.-O.; Bracarda, S.; et al. Nivolumab in Metastatic Urothelial Carcinoma after Platinum Therapy (CheckMate 275): A Multicentre, Single-Arm, Phase 2 Trial. Lancet Oncol. 2017, 18, 312-322. [CrossRef]

45. Overman, M.J.; McDermott, R.; Leach, J.L.; Lonardi, S.; Lenz, H.-J.; Morse, M.A.; Desai, J.; Hill, A.; Axelson, M.; Moss, R.A.; et al. Nivolumab in Patients with Metastatic DNA Mismatch Repair-Deficient or Microsatellite Instability-High Colorectal Cancer (CheckMate 142): An Open-Label, Multicentre, Phase 2 Study. Lancet Oncol. 2017, 18, 1182-1191. [CrossRef]

46. Ferris, R.L.; Blumenschein, G.; Fayette, J.; Guigay, J.; Colevas, A.D.; Licitra, L.; Harrington, K.; Kasper, S.; Vokes, E.E.; Even, C.; et al. Nivolumab for Recurrent Squamous-Cell Carcinoma of the Head and Neck. N. Engl. J. Med. 2016, 375, 1856-1867. [CrossRef]

47. El-Khoueiry, A.B.; Sangro, B.; Yau, T.; Crocenzi, T.S.; Kudo, M.; Hsu, C.; Kim, T.-Y.; Choo, S.-P.; Trojan, J.; Welling, T.H.; et al. Nivolumab in Patients with Advanced Hepatocellular Carcinoma (CheckMate 040): An Open-Label, Non-Comparative, Phase 1/2 Dose Escalation and Expansion Trial. Lancet Lond. Engl. 2017, 389, 2492-2502. [CrossRef]

48. Green, M.R.; Monti, S.; Rodig, S.J.; Juszczynski, P.; Currie, T.; O’Donnell, E.; Chapuy, B.; Takeyama, K.; Neuberg, D.; Golub, T.R.; et al. Integrative Analysis Reveals Selective 9p24.1 Amplification, Increased PD-1 Ligand Expression, and Further Induction via JAK2 in Nodular Sclerosing Hodgkin Lymphoma and Primary Mediastinal Large B-Cell Lymphoma. Blood 2010, 116, 3268-3277. [CrossRef]

49. Ansell, S.M.; Lesokhin, A.M.; Borrello, I.; Halwani, A.; Scott, E.C.; Gutierrez, M.; Schuster, S.J.; Millenson, M.M.; Cattry, D.; Freeman, G.J.; et al. PD-1 Blockade with Nivolumab in Relapsed or Refractory Hodgkin's Lymphoma. N. Engl. J. Med. 2015, 372, 311-319. [CrossRef]

50. Younes, A.; Santoro, A.; Shipp, M.; Zinzani, P.L.; Timmerman, J.M.; Ansell, S.; Armand, P.; Fanale, M.; Ratanatharathorn, V.; Kuruvilla, J.; et al. Nivolumab for Classical Hodgkin's Lymphoma after Failure of Both Autologous Stem-Cell Transplantation and Brentuximab Vedotin: A Multicentre, Multicohort, Single-Arm Phase 2 Trial. Lancet Oncol. 2016, 17, 1283-1294. [CrossRef]

51. Weber, J.S.; D'Angelo, S.P.; Minor, D.; Hodi, F.S.; Gutzmer, R.; Neyns, B.; Hoeller, C.; Khushalani, N.I.; Miller, W.H.; Lao, C.D.; et al. Nivolumab versus Chemotherapy in Patients with Advanced Melanoma Who Progressed after Anti-CTLA-4 Treatment (CheckMate 037): A Randomised, Controlled, Open-Label, Phase 3 Trial. Lancet Oncol. 2015, 16, 375-384. [CrossRef]

52. Brahmer, J.; Reckamp, K.L.; Baas, P.; Crinò, L.; Eberhardt, W.E.E.; Poddubskaya, E.; Antonia, S.; Pluzanski, A.; Vokes, E.E.; Holgado, E.; et al. Nivolumab versus Docetaxel in Advanced Squamous-Cell Non-Small-Cell Lung Cancer. N. Engl. J. Med. 2015, 373, 123-135. [CrossRef] [PubMed]

53. Borghaei, H.; Paz-Ares, L.; Horn, L.; Spigel, D.R.; Steins, M.; Ready, N.E.; Chow, L.Q.; Vokes, E.E.; Felip, E.; Holgado, E.; et al. Nivolumab versus Docetaxel in Advanced Nonsquamous Non-Small-Cell Lung Cancer. N. Engl. J. Med. 2015, 373, 1627-1639. [CrossRef] [PubMed] 
54. Vokes, E.E.; Ready, N.; Felip, E.; Horn, L.; Burgio, M.A.; Antonia, S.J.; Frontera, O.A.; Gettinger, S.; Holgado, E.; Spigel, D.; et al. Nivolumab versus Docetaxel in Previously Treated Advanced Non-Small-Cell Lung Cancer (CheckMate 017 and CheckMate 057): 3-Year Update and Outcomes in Patients with Liver Metastases. Ann. Oncol. 2018, 29, 959-965. [CrossRef] [PubMed]

55. Motzer, R.J.; Escudier, B.; McDermott, D.F.; George, S.; Hammers, H.J.; Srinivas, S.; Tykodi, S.S.; Sosman, J.A.; Procopio, G.; Plimack, E.R.; et al. Nivolumab versus Everolimus in Advanced Renal-Cell Carcinoma. N. Engl. J. Med. 2015, 373, 1803-1813. [CrossRef] [PubMed]

56. Antonia, S.J.; López-Martin, J.A.; Bendell, J.; Ott, P.A.; Taylor, M.; Eder, J.P.; Jäger, D.; Pietanza, M.C.; Le, D.T.; De Braud, F.; et al. Nivolumab Alone and Nivolumab plus Ipilimumab in Recurrent Small-Cell Lung Cancer (CheckMate 032): A Multicentre, Open-Label, Phase 1/2 Trial. Lancet Oncol. 2016, 17, 883-895. [CrossRef]

57. Chung, H.C.; Ros, W.; Delord, J.-P.; Perets, R.; Italiano, A.; Shapira-Frommer, R.; Manzuk, L.; Piha-Paul, S.A.; Xu, L.; Zeigenfuss, S.; et al. Efficacy and Safety of Pembrolizumab in Previously Treated Advanced Cervical Cancer: Results from the Phase II KEYNOTE-158 Study. J. Clin. Oncol. 2019, 37, 1470-1478. [CrossRef]

58. Fuchs, C.S.; Doi, T.; Jang, R.W.; Muro, K.; Satoh, T.; Machado, M.; Sun, W.; Jalal, S.I.; Shah, M.A.; Metges, J.-P.; et al. Safety and Efficacy of Pembrolizumab Monotherapy in Patients with Previously Treated Advanced Gastric and Gastroesophageal Junction Cancer: Phase 2 Clinical KEYNOTE-059 Trial. JAMA Oncol. 2018, 4, e180013. [CrossRef]

59. Seiwert, T.Y.; Burtness, B.; Mehra, R.; Weiss, J.; Berger, R.; Eder, J.P.; Heath, K.; McClanahan, T.; Lunceford, J.; Gause, C.; et al. Safety and Clinical Activity of Pembrolizumab for Treatment of Recurrent or Metastatic Squamous Cell Carcinoma of the Head and Neck (KEYNOTE-012): An Open-Label, Multicentre, Phase 1b Trial. Lancet Oncol. 2016, 17, 956-965. [CrossRef]

60. Cohen, E.E.W.; Soulières, D.; Tourneau, C.L.; Dinis, J.; Licitra, L.; Ahn, M.-J.; Soria, A.; Machiels, J.-P.; Mach, N.; Mehra, R.; et al. Pembrolizumab versus Methotrexate, Docetaxel, or Cetuximab for Recurrent or Metastatic Head-and-Neck Squamous Cell Carcinoma (KEYNOTE-040): A Randomised, Open-Label, Phase 3 Study. Lancet 2019, 393, 156-167. [CrossRef]

61. Burtness, B.; Harrington, K.J.; Greil, R.; Soulières, D.; Tahara, M.; De Castro, G.; Psyrri, A.; Baste Rotllan, N.; Neupane, P.C.; Bratland, Å.; et al. LBA8_PRKEYNOTE-048: Phase III Study of First-Line Pembrolizumab (P) for Recurrent/Metastatic Head and Neck Squamous Cell Carcinoma (R/M HNSCC). Ann. Oncol. 2018, 29 (Suppl. 8). [CrossRef]

62. Zhu, A.X.; Finn, R.S.; Edeline, J.; Cattan, S.; Ogasawara, S.; Palmer, D.; Verslype, C.; Zagonel, V.; Fartoux, L.; Vogel, A.; et al. Pembrolizumab in Patients with Advanced Hepatocellular Carcinoma Previously Treated with Sorafenib (KEYNOTE-224): A Non-Randomised, Open-Label Phase 2 Trial. Lancet Oncol. 2018, 19, 940-952. [CrossRef]

63. Chen, R.; Zinzani, P.L.; Fanale, M.A.; Armand, P.; Johnson, N.A.; Brice, P.; Radford, J.; Ribrag, V.; Molin, D.; Vassilakopoulos, T.P.; et al. Phase II Study of the Efficacy and Safety of Pembrolizumab for Relapsed/Refractory Classic Hodgkin Lymphoma. J. Clin. Oncol. 2017, 35, 2125-2132. [CrossRef] [PubMed]

64. Garon, E.B.; Rizvi, N.A.; Hui, R.; Leighl, N.; Balmanoukian, A.S.; Eder, J.P.; Patnaik, A.; Aggarwal, C.; Gubens, M.; Horn, L.; et al. Pembrolizumab for the Treatment of Non-Small-Cell Lung Cancer. N. Engl. J. Med. 2015, 372, 2018-2028. [CrossRef] [PubMed]

65. Robert, C.; Ribas, A.; Wolchok, J.D.; Hodi, F.S.; Hamid, O.; Kefford, R.; Weber, J.S.; Joshua, A.M.; Hwu, W.-J.; Gangadhar, T.C.; et al. Anti-Programmed-Death-Receptor-1 Treatment with Pembrolizumab in Ipilimumab-Refractory Advanced Melanoma: A Randomised Dose-Comparison Cohort of a Phase 1 Trial. Lancet Lond. Engl. 2014, 384, 1109-1117. [CrossRef]

66. Ribas, A.; Puzanov, I.; Dummer, R.; Schadendorf, D.; Hamid, O.; Robert, C.; Hodi, F.S.; Schachter, J.; Pavlick, A.C.; Lewis, K.D.; et al. Pembrolizumab versus Investigator-Choice Chemotherapy for Ipilimumab-Refractory Melanoma (KEYNOTE-002): A Randomised, Controlled, Phase 2 Trial. Lancet Oncol. 2015, 16, 908-918. [CrossRef]

67. Robert, C.; Schachter, J.; Long, G.V.; Arance, A.; Grob, J.J.; Mortier, L.; Daud, A.; Carlino, M.S.; McNeil, C.; Lotem, M.; et al. Pembrolizumab versus Ipilimumab in Advanced Melanoma. N. Engl. J. Med. 2015, 372, 2521-2532. [CrossRef]

68. Eggermont, A.M.M.; Blank, C.U.; Mandala, M.; Long, G.V.; Atkinson, V.; Dalle, S.; Haydon, A.; Lichinitser, M.; Khattak, A.; Carlino, M.S.; et al. Adjuvant Pembrolizumab versus Placebo in Resected Stage III Melanoma. N. Engl. J. Med. 2018. [CrossRef] 
69. Nghiem, P.; Bhatia, S.; Lipson, E.J.; Sharfman, W.H.; Kudchadkar, R.R.; Brohl, A.S.; Friedlander, P.A.; Daud, A.; Kluger, H.M.; Reddy, S.A.; et al. Durable Tumor Regression and Overall Survival in Patients with Advanced Merkel Cell Carcinoma Receiving Pembrolizumab as First-Line Therapy. J. Clin. Oncol. 2019, 37, 693-702. [CrossRef]

70. Pembrolizumab in MSI-H or dMMR Solid Tumors: 'First Tissue/Site-Agnostic' Approval by FDA - The ASCO Post. Available online: https://www.ascopost.com/issues/february-10-2018/pembrolizumab-in-msi-hor-dmmr-solid-tumors-first-tissuesite-agnostic-approval-by-fda/ (accessed on 11 August 2019).

71. Armand, P.; Rodig, S.J.; Melnichenko, V.; Thieblemont, C.; Bouabdallah, K.; Tumyan, G.; Özcan, M.; Portino, S.; Fogliatto, L.; Caballero, D.; et al. Pembrolizumab in Patients with Relapsed or Refractory Primary Mediastinal Large B-Cell Lymphoma (PMBCL): Data from the Keynote-013 and Keynote-170 Studies. Blood 2018, 132 (Suppl. 1), 228. [CrossRef]

72. Atkins, M.B.; Plimack, E.R.; Puzanov, I.; Fishman, M.N.; McDermott, D.F.; Cho, D.C.; Vaishampayan, U.; George, S.; Olencki, T.E.; Tarazi, J.C.; et al. Axitinib in Combination with Pembrolizumab in Patients with Advanced Renal Cell Cancer: A Non-Randomised, Open-Label, Dose-Finding, and Dose-Expansion Phase 1b Trial. Lancet Oncol. 2018, 19, 405-415. [CrossRef]

73. Rini, B.I.; Plimack, E.R.; Stus, V.; Gafanov, R.; Hawkins, R.; Nosov, D.; Pouliot, F.; Alekseev, B.; Soulières, D.; Melichar, B.; et al. Pembrolizumab plus Axitinib versus Sunitinib for Advanced Renal-Cell Carcinoma. N. Engl. J. Med. 2019, 380, 1116-1127. [CrossRef] [PubMed]

74. Rosenberg, J.E.; Hoffman-Censits, J.; Powles, T.; van der Heijden, M.S.; Balar, A.V.; Necchi, A.; Dawson, N.; O'Donnell, P.H.; Balmanoukian, A.; Loriot, Y.; et al. Atezolizumab in Patients with Locally Advanced and Metastatic Urothelial Carcinoma Who Have Progressed Following Treatment with Platinum-Based Chemotherapy: A Single-Arm, Multicentre, Phase 2 Trial. Lancet 2016, 387, 1909-1920. [CrossRef]

75. Powles, T.; Durán, I.; van der Heijden, M.S.; Loriot, Y.; Vogelzang, N.J.; De Giorgi, U.; Oudard, S.; Retz, M.M.; Castellano, D.; Bamias, A.; et al. Atezolizumab versus Chemotherapy in Patients with Platinum-Treated Locally Advanced or Metastatic Urothelial Carcinoma (IMvigor211): A Multicentre, Open-Label, Phase 3 Randomised Controlled Trial. Lancet 2018, 391, 748-757. [CrossRef]

76. Schmid, P.; Adams, S.; Rugo, H.S.; Schneeweiss, A.; Barrios, C.H.; Iwata, H.; Diéras, V.; Hegg, R.; Im, S.-A.; Shaw Wright, G.; et al. Atezolizumab and Nab-Paclitaxel in Advanced Triple-Negative Breast Cancer. N. Engl. J. Med. 2018, 379, 2108-2121. [CrossRef]

77. Fehrenbacher, L.; Spira, A.; Ballinger, M.; Kowanetz, M.; Vansteenkiste, J.; Mazieres, J.; Park, K.; Smith, D.; Artal-Cortes, A.; Lewanski, C.; et al. Atezolizumab versus Docetaxel for Patients with Previously Treated Non-Small-Cell Lung Cancer (POPLAR): A Multicentre, Open-Label, Phase 2 Randomised Controlled Trial. Lancet 2016, 387, 1837-1846. [CrossRef]

78. Socinski, M.A.; Jotte, R.M.; Cappuzzo, F.; Orlandi, F.; Stroyakovskiy, D.; Nogami, N.; Rodríguez-Abreu, D.; Moro-Sibilot, D.; Thomas, C.A.; Barlesi, F.; et al. Atezolizumab for First-Line Treatment of Metastatic Nonsquamous NSCLC. N. Engl. J. Med. 2018, 378, 2288-2301. [CrossRef] [PubMed]

79. Horn, L.; Mansfield, A.S.; Szczęsna, A.; Havel, L.; Krzakowski, M.; Hochmair, M.J.; Huemer, F.; Losonczy, G.; Johnson, M.L.; Nishio, M.; et al. First-Line Atezolizumab plus Chemotherapy in Extensive-Stage Small-Cell Lung Cancer. N. Engl. J. Med. 2018, 379, 2220-2229. [CrossRef]

80. Apolo, A.B.; Infante, J.R.; Balmanoukian, A.; Patel, M.R.; Wang, D.; Kelly, K.; Mega, A.E.; Britten, C.D.; Ravaud, A.; Mita, A.C.; et al. Avelumab, an Anti-Programmed Death-Ligand 1 Antibody, In Patients with Refractory Metastatic Urothelial Carcinoma: Results from a Multicenter, Phase Ib Study. J. Clin. Oncol. 2017, 35, 2117-2124. [CrossRef]

81. Kaufman, H.L.; Russell, J.; Hamid, O.; Bhatia, S.; Terheyden, P.; D’Angelo, S.P.; Shih, K.C.; Lebbé, C.; Linette, G.P.; Milella, M.; et al. Avelumab in Patients with Chemotherapy-Refractory Metastatic Merkel Cell Carcinoma: A Multicentre, Single-Group, Open-Label, Phase 2 Trial. Lancet Oncol. 2016, 17, 1374-1385. [CrossRef]

82. Motzer, R.J.; Penkov, K.; Haanen, J.; Rini, B.; Albiges, L.; Campbell, M.T.; Venugopal, B.; Kollmannsberger, C.; Negrier, S.; Uemura, M.; et al. Avelumab plus Axitinib versus Sunitinib for Advanced Renal-Cell Carcinoma. N. Engl. J. Med. 2019, 380, 1103-1115. [CrossRef] 
83. Massard, C.; Gordon, M.S.; Sharma, S.; Rafii, S.; Wainberg, Z.A.; Luke, J.; Curiel, T.J.; Colon-Otero, G.; Hamid, O.; Sanborn, R.E.; et al. Safety and Efficacy of Durvalumab (MEDI4736), an Anti-Programmed Cell Death Ligand-1 Immune Checkpoint Inhibitor, in Patients with Advanced Urothelial Bladder Cancer. J. Clin. Oncol. 2016, 34, 3119-3125. [CrossRef] [PubMed]

84. Antonia, S.J.; Villegas, A.; Daniel, D.; Vicente, D.; Murakami, S.; Hui, R.; Yokoi, T.; Chiappori, A.; Lee, K.H.; De Wit, M.; et al. Durvalumab after Chemoradiotherapy in Stage III Non-Small-Cell Lung Cancer. N. Engl. J. Med. 2017, 377, 1919-1929. [CrossRef] [PubMed]

85. Lim, M.; Ye, X.; Piotrowski, A.F.; Desai, A.S.; Ahluwalia, M.S.; Walbert, T.; Fisher, J.D.; Desideri, S.; Belcaid, Z.; Jackson, C.; et al. Updated Phase I Trial of Anti-LAG-3 or Anti-CD137 Alone and in Combination with Anti-PD-1 in Patients with Recurrent GBM. J. Clin. Oncol. 2019, 37 (Suppl. 15), 2017. [CrossRef]

86. Kluger, H.; Weiss, S.A.; Olszanski, A.J.; Schuchter, L.; Linette, G.P.; Garland, L.; Iannotti, N.O.; Johnson, M.; Avsar, E.; Srivastava, M.K.; et al. Abstract CT089: Phase Ib/II of CD40 Agonistic Antibody APX005M in Combination with Nivolumab (Nivo) in Subjects with Metastatic Melanoma (M) or Non-Small Cell Lung Cancer (NSCLC). Cancer Res. 2019, 79 (Suppl. 13), CT089. [CrossRef]

87. O'Hara, M.H.; O’Reilly, E.M.; Rosemarie, M.; Varadhachary, G.; Wainberg, Z.A.; Ko, A.; Fisher, G.A.; Rahma, O.; Lyman, J.P.; Cabanski, C.R.; et al. Abstract CT004: A Phase Ib Study of CD40 Agonistic Monoclonal Antibody APX005M Together with Gemcitabine (Gem) and Nab-Paclitaxel (NP) with or without Nivolumab (Nivo) in Untreated Metastatic Ductal Pancreatic Adenocarcinoma (PDAC) Patients. Cancer Res. 2019, 79 (Suppl. 13), CT004. [CrossRef]

88. Bajor, D.L.; Mick, R.; Riese, M.J.; Richman, L.P.; Xu, X.; Torigian, D.A.; Stelekati, E.; Sweeney, M.; Sullivan, B.; Schuchter, L.M.; et al. Abstract CT137: Combination of Agonistic CD40 Monoclonal Antibody CP-870,893 and Anti-CTLA-4 Antibody Tremelimumab in Patients with Metastatic Melanoma. Cancer Res. 2015, 75 (Suppl. 15), CT137. [CrossRef]

89. Beatty, G.L.; Torigian, D.A.; Chiorean, E.G.; Saboury, B.; Brothers, A.; Alavi, A.; Troxel, A.B.; Sun, W.; Teitelbaum, U.R.; Vonderheide, R.H.; et al. A Phase I Study of an Agonist CD40 Monoclonal Antibody (CP-870,893) in Combination with Gemcitabine in Patients with Advanced Pancreatic Ductal Adenocarcinoma. Clin. Cancer Res. 2013, 19, 6286-6295. [CrossRef]

90. Linch, S.N.; McNamara, M.J.; Redmond, W.L. OX40 Agonists and Combination Immunotherapy: Putting the Pedal to the Metal. Front. Oncol. 2015, 5, 34. [CrossRef]

91. Redmond, W.L.; Linch, S.N.; Kasiewicz, M.J. Combined Targeting of Costimulatory (OX40) and Coinhibitory (CTLA-4) Pathways Elicits Potent Effector T Cells Capable of Driving Robust Antitumor Immunity. Cancer Immunol. Res. 2014, 2, 142-153. [CrossRef]

92. Curti, B.D.; Kovacsovics-Bankowski, M.; Morris, N.; Walker, E.; Chisholm, L.; Floyd, K.; Walker, J.; Gonzalez, I.; Meeuwsen, T.; Fox, B.A.; et al. OX40 Is a Potent Immune Stimulating Target in Late Stage Cancer Patients. Cancer Res. 2013, 73, 7189-7198. [CrossRef]

93. Tang, J.; Yu, J.X.; Hubbard-Lucey, V.M.; Neftelinov, S.T.; Hodge, J.P.; Lin, Y. Trial Watch: The Clinical Trial Landscape for PD1/PDL1 Immune Checkpoint Inhibitors. Nat. Rev. Drug Discov. 2018, 17, 854-855. [CrossRef] [PubMed]

94. Makker, V.; Rasco, D.; Vogelzang, N.J.; Brose, M.S.; Cohn, A.L.; Mier, J.; Di Simone, C.; Hyman, D.M.; Stepan, D.E.; Dutcus, C.E.; et al. Lenvatinib plus Pembrolizumab in Patients with Advanced Endometrial Cancer: An Interim Analysis of a Multicentre, Open-Label, Single-Arm, Phase 2 Trial. Lancet Oncol. 2019, 20 , 711-718. [CrossRef]

95. Pitt, J.M.; Vétizou, M.; Daillère, R.; Roberti, M.P.; Yamazaki, T.; Routy, B.; Lepage, P.; Boneca, I.G.; Chamaillard, M.; Kroemer, G.; et al. Resistance Mechanisms to Immune-Checkpoint Blockade in Cancer: Tumor-Intrinsic and -Extrinsic Factors. Immunity 2016, 44, 1255-1269. [CrossRef] [PubMed]

96. O’Donnell, J.S.; Long, G.V.; Scolyer, R.A.; Teng, M.W.L.; Smyth, M.J. Resistance to PD1/PDL1 Checkpoint Inhibition. Cancer Treat. Rev. 2017, 52, 71-81. [CrossRef] [PubMed]

97. Sharma, P.; Hu-Lieskovan, S.; Wargo, J.A.; Ribas, A. Primary, Adaptive, and Acquired Resistance to Cancer Immunotherapy. Cell 2017, 168, 707-723. [CrossRef] [PubMed]

98. Jenkins, R.W.; Barbie, D.A.; Flaherty, K.T. Mechanisms of Resistance to Immune Checkpoint Inhibitors. Br. J. Cancer 2018, 118, 9-16. [CrossRef] 
99. Gubin, M.M.; Zhang, X.; Schuster, H.; Caron, E.; Ward, J.P.; Noguchi, T.; Ivanova, Y.; Hundal, J.; Arthur, C.D.; Krebber, W.-J.; et al. Checkpoint Blockade Cancer Immunotherapy Targets Tumour-Specific Mutant Antigens. Nature 2014, 515, 577-581. [CrossRef]

100. Marincola, F.M.; Jaffee, E.M.; Hicklin, D.J.; Ferrone, S. Escape of Human Solid Tumors from T-Cell Recognition: Molecular Mechanisms and Functional Significance. Adv. Immunol. 2000, 74, 181-273.

101. Sucker, A.; Zhao, F.; Real, B.; Heeke, C.; Bielefeld, N.; Maßen, S.; Horn, S.; Moll, I.; Maltaner, R.; Horn, P.A.; et al. Genetic Evolution of T-Cell Resistance in the Course of Melanoma Progression. Clin. Cancer Res. 2014, 20, 6593-6604. [CrossRef]

102. Liu, C.; Peng, W.; Xu, C.; Lou, Y.; Zhang, M.; Wargo, J.A.; Chen, J.Q.; Li, H.S.; Watowich, S.S.; Yang, Y.; et al. BRAF Inhibition Increases Tumor Infiltration by T Cells and Enhances the Antitumor Activity of Adoptive Immunotherapy in Mice. Clin. Cancer Res. 2013, 19, 393-403. [CrossRef]

103. Spranger, S.; Bao, R.; Gajewski, T.F. Melanoma-Intrinsic $\beta$-Catenin Signalling Prevents Anti-Tumour Immunity. Nature 2015, 523, 231-235. [CrossRef] [PubMed]

104. Shin, D.S.; Zaretsky, J.M.; Escuin-Ordinas, H.; Garcia-Diaz, A.; Hu-Lieskovan, S.; Kalbasi, A.; Grasso, C.S.; Hugo, W.; Sandoval, S.; Torrejon, D.Y.; et al. Primary Resistance to PD-1 Blockade Mediated by JAK1/2 Mutations. Cancer Discov. 2017, 7, 188-201. [CrossRef] [PubMed]

105. Kryczek, I.; Zou, L.; Rodriguez, P.; Zhu, G.; Wei, S.; Mottram, P.; Brumlik, M.; Cheng, P.; Curiel, T.; Myers, L.; et al. B7-H4 Expression Identifies a Novel Suppressive Macrophage Population in Human Ovarian Carcinoma. J. Exp. Med. 2006, 203, 871-881. [CrossRef] [PubMed]

106. Kuang, D.-M.; Zhao, Q.; Peng, C.; Xu, J.; Zhang, J.-P.; Wu, C.; Zheng, L. Activated Monocytes in Peritumoral Stroma of Hepatocellular Carcinoma Foster Immune Privilege and Disease Progression through PD-L1. J. Exp. Med. 2009, 206, 1327-1337. [CrossRef] [PubMed]

107. Meyer, C.; Cagnon, L.; Costa-Nunes, C.M.; Baumgaertner, P.; Montandon, N.; Leyvraz, L.; Michielin, O.; Romano, E.; Speiser, D.E. Frequencies of Circulating MDSC Correlate with Clinical Outcome of Melanoma Patients Treated with Ipilimumab. Cancer Immunol. Immunother. 2014, 63, 247-257. [CrossRef] [PubMed]

108. Marelli, G.; Howells, A.; Lemoine, N.R.; Wang, Y. Oncolytic Viral Therapy and the Immune System: A Double-Edged Sword Against Cancer. Front. Immunol. 2018, 9, 866. [CrossRef] [PubMed]

109. Andtbacka, R.H.I.; Kaufman, H.L.; Collichio, F.; Amatruda, T.; Senzer, N.; Chesney, J.; Delman, K.A.; Spitler, L.E.; Puzanov, I.; Agarwala, S.S.; et al. Talimogene Laherparepvec Improves Durable Response Rate in Patients with Advanced Melanoma. J. Clin. Oncol. 2015, 33, 2780-2788. [CrossRef]

110. Ribas, A.; Dummer, R.; Puzanov, I.; VanderWalde, A.; Andtbacka, R.H.I.; Michielin, O.; Olszanski, A.J.; Malvehy, J.; Cebon, J.; Fernandez, E.; et al. Oncolytic Virotherapy Promotes Intratumoral T Cell Infiltration and Improves Anti-PD-1 Immunotherapy. Cell 2018, 174, 1031-1032. [CrossRef]

111. Schouppe, E.; De Baetselier, P.; Van Ginderachter, J.A.; Sarukhan, A. Instruction of Myeloid Cells by the Tumor Microenvironment. Oncoimmunology 2012, 1, 1135-1145. [CrossRef]

112. Liu, T.; Han, C.; Wang, S.; Fang, P.; Ma, Z.; Xu, L.; Yin, R. Cancer-Associated Fibroblasts: An Emerging Target of Anti-Cancer Immunotherapy. J. Hematol. Oncol. 2019, 12, 86. [CrossRef]

113. Awad, R.M.; De Vlaeminck, Y.; Maebe, J.; Goyvaerts, C.; Breckpot, K. Turn Back the TIMe: Targeting Tumor Infiltrating Myeloid Cells to Revert Cancer Progression. Front. Immunol. 2018, 9, 1977. [CrossRef] [PubMed]

114. Shurin, G.V.; Ouellette, C.E.; Shurin, M.R. Regulatory Dendritic Cells in the Tumor Immunoenvironment. Cancer Immunol. Immunother. 2012, 61, 223-230. [CrossRef] [PubMed]

115. Fridlender, Z.G.; Sun, J.; Kim, S.; Kapoor, V.; Cheng, G.; Ling, L.; Worthen, G.S.; Albelda, S.M. Polarization of Tumor-Associated Neutrophil Phenotype by TGF-Beta: “N1" versus “N2" TAN. Cancer Cell 2009, 16, 183-194. [CrossRef]

116. Ma, Y.; Aymeric, L.; Locher, C.; Kroemer, G.; Zitvogel, L. The Dendritic Cell-Tumor Cross-Talk in Cancer. Curr. Opin. Immunol. 2011, 23, 146-152. [CrossRef]

117. Ostrand-Rosenberg, S.; Sinha, P. Myeloid-Derived Suppressor Cells: Linking Inflammation and Cancer. J. Immunol. 2009, 182, 4499-4506. [CrossRef] [PubMed]

118. Burke, B.; Giannoudis, A.; Corke, K.P.; Gill, D.; Wells, M.; Ziegler-Heitbrock, L.; Lewis, C.E. Hypoxia-Induced Gene Expression in Human Macrophages: Implications for Ischemic Tissues and Hypoxia-Regulated Gene Therapy. Am. J. Pathol. 2003, 163, 1233-1243. [CrossRef] 
119. Corzo, C.A.; Condamine, T.; Lu, L.; Cotter, M.J.; Youn, J.-I.; Cheng, P.; Cho, H.-I.; Celis, E.; Quiceno, D.G.; Padhya, T.; et al. HIF-1 $\alpha$ Regulates Function and Differentiation of Myeloid-Derived Suppressor Cells in the Tumor Microenvironment. J. Exp. Med. 2010, 207, 2439-2453. [CrossRef]

120. Mahadevan, N.R.; Zanetti, M. Tumor Stress inside out: Cell-Extrinsic Effects of the Unfolded Protein Response in Tumor Cells Modulate the Immunological Landscape of the Tumor Microenvironment. J. Immunol. 2011, 187, 4403-4409. [CrossRef]

121. Kalinski, P. Regulation of Immune Responses by Prostaglandin E2. J. Immunol. 2012, 188, 21-28. [CrossRef]

122. Sato, T.; Terai, M.; Tamura, Y.; Alexeev, V.; Mastrangelo, M.J.; Selvan, S.R. Interleukin 10 in the Tumor Microenvironment: A Target for Anticancer Immunotherapy. Immunol. Res. 2011, 51, 170-182. [CrossRef]

123. Flavell, R.A.; Sanjabi, S.; Wrzesinski, S.H.; Licona-Limón, P. The Polarization of Immune Cells in the Tumour Environment by TGFbeta. Nat. Rev. Immunol. 2010, 10, 554-567. [CrossRef] [PubMed]

124. Ahmadzadeh, M.; Rosenberg, S.A. TGF-Beta 1 Attenuates the Acquisition and Expression of Effector Function by Tumor Antigen-Specific Human Memory CD8 T Cells. J. Immunol. 2005, 174, 5215-5223. [CrossRef] [PubMed]

125. Wang, C.; Thudium, K.B.; Han, M.; Wang, X.-T.; Huang, H.; Feingersh, D.; Garcia, C.; Wu, Y.; Kuhne, M.; Srinivasan, M.; et al. In Vitro Characterization of the Anti-PD-1 Antibody Nivolumab, BMS-936558, and In Vivo Toxicology in Non-Human Primates. Cancer Immunol. Res. 2014, 2, 846-856. [CrossRef] [PubMed]

126. Fessas, P.; Lee, H.; Ikemizu, S.; Janowitz, T. A Molecular and Preclinical Comparison of the PD-1-Targeted T-Cell Checkpoint Inhibitors Nivolumab and Pembrolizumab. Semin. Oncol. 2017, 44, 136-140. [CrossRef]

127. Overman, M.J.; Lonardi, S.; Wong, K.Y.M.; Lenz, H.-J.; Gelsomino, F.; Aglietta, M.; Morse, M.A.; Van Cutsem, E.; McDermott, R.; Hill, A.; et al. Durable Clinical Benefit with Nivolumab Plus Ipilimumab in DNA Mismatch Repair-Deficient/Microsatellite Instability-High Metastatic Colorectal Cancer. J. Clin. Oncol. 2018, 36, 773-779. [CrossRef]

128. Postow, M.A.; Chesney, J.; Pavlick, A.C.; Robert, C.; Grossmann, K.; McDermott, D.; Linette, G.P.; Meyer, N.; Giguere, J.K.; Agarwala, S.S.; et al. Nivolumab and Ipilimumab versus Ipilimumab in Untreated Melanoma. N. Engl. J. Med. 2015, 372, 2006-2017. [CrossRef]

129. Larkin, J.; Chiarion-Sileni, V.; Gonzalez, R.; Grob, J.J.; Cowey, C.L.; Lao, C.D.; Schadendorf, D.; Dummer, R.; Smylie, M.; Rutkowski, P.; et al. Combined Nivolumab and Ipilimumab or Monotherapy in Untreated Melanoma. N. Engl. J. Med. 2015, 373, 23-34. [CrossRef]

130. Eggermont, A.M.M.; Chiarion-Sileni, V.; Grob, J.-J.; Dummer, R.; Wolchok, J.D.; Schmidt, H.; Hamid, O.; Robert, C.; Ascierto, P.A.; Richards, J.M.; et al. Adjuvant Ipilimumab versus Placebo after Complete Resection of High-Risk Stage III Melanoma (EORTC 18071): A Randomised, Double-Blind, Phase 3 Trial. Lancet Oncol. 2015, 16, 522-530. [CrossRef]

131. Merchant, M.S.; Wright, M.; Baird, K.; Wexler, L.H.; Rodriguez-Galindo, C.; Bernstein, D.; Delbrook, C.; Lodish, M.; Bishop, R.; Wolchok, J.D.; et al. Phase I Clinical Trial of Ipilimumab in Pediatric Patients with Advanced Solid Tumors. Clin. Cancer Res. 2016, 22, 1364-1370. [CrossRef]

132. Geoerger, B.; Bergeron, C.; Gore, L.; Sender, L.; Dunkel, I.J.; Herzog, C.; Brochez, L.; Cruz, O.; Nysom, K.; Berghorn, E.; et al. Phase II Study of Ipilimumab in Adolescents with Unresectable Stage III or IV Malignant Melanoma. Eur. J. Cancer 2017, 86, 358-363. [CrossRef]

133. Long, G.V.; Atkinson, V.; Lo, S.; Sandhu, S.; Guminski, A.D.; Brown, M.P.; Wilmott, J.S.; Edwards, J.; Gonzalez, M.; Scolyer, R.A.; et al. Combination Nivolumab and Ipilimumab or Nivolumab Alone in Melanoma Brain Metastases: A Multicentre Randomised Phase 2 Study. Lancet Oncol. 2018, 19, 672-681. [CrossRef]

134. Hellmann, M.D.; Ciuleanu, T.-E.; Pluzanski, A.; Lee, J.S.; Otterson, G.A.; Audigier-Valette, C.; Minenza, E.; Linardou, H.; Burgers, S.; Salman, P.; et al. Nivolumab plus Ipilimumab in Lung Cancer with a High Tumor Mutational Burden. N. Engl. J. Med. 2018, 378, 2093-2104. [CrossRef] [PubMed]

135. Slovin, S.F.; Higano, C.S.; Hamid, O.; Tejwani, S.; Harzstark, A.; Alumkal, J.J.; Scher, H.I.; Chin, K.; Gagnier, P.; McHenry, M.B.; et al. Ipilimumab Alone or in Combination with Radiotherapy in Metastatic Castration-Resistant Prostate Cancer: Results from an Open-Label, Multicenter Phase I/II Study. Ann. Oncol. 2013, 24, 1813-1821. [CrossRef] 
136. Reck, M.; Bondarenko, I.; Luft, A.; Serwatowski, P.; Barlesi, F.; Chacko, R.; Sebastian, M.; Lu, H.; Cuillerot, J.-M.; Lynch, T.J. Ipilimumab in Combination with Paclitaxel and Carboplatin as First-Line Therapy in Extensive-Disease-Small-Cell Lung Cancer: Results from a Randomized, Double-Blind, Multicenter Phase 2 Trial. Ann. Oncol. 2013, 24, 75-83. [CrossRef] [PubMed]

137. Reck, M.; Luft, A.; Szczesna, A.; Havel, L.; Kim, S.-W.; Akerley, W.; Pietanza, M.C.; Wu, Y.; Zielinski, C.; Thomas, M.; et al. Phase III Randomized Trial of Ipilimumab Plus Etoposide and Platinum Versus Placebo Plus Etoposide and Platinum in Extensive-Stage Small-Cell Lung Cancer. J. Clin. Oncol. 2016, 34, 3740-3748. [CrossRef] [PubMed]

138. Ribas, A.; Kefford, R.; Marshall, M.A.; Punt, C.J.A.; Haanen, J.B.; Marmol, M.; Garbe, C.; Gogas, H.; Schachter, J.; Linette, G.; et al. Phase III Randomized Clinical Trial Comparing Tremelimumab With Standard-of-Care Chemotherapy in Patients with Advanced Melanoma. J. Clin. Oncol. 2013, 31, 616-622. [CrossRef]

139. Calabrò, L.; Morra, A.; Fonsatti, E.; Cutaia, O.; Fazio, C.; Annesi, D.; Lenoci, M.; Amato, G.; Danielli, R.; Altomonte, M.; et al. Efficacy and Safety of an Intensified Schedule of Tremelimumab for Chemotherapy-Resistant Malignant Mesothelioma: An Open-Label, Single-Arm, Phase 2 Study. Lancet Respir. Med. 2015, 3, 301-309. [CrossRef]

140. Rizvi, N.A.; Chul Cho, B.; Reinmuth, N.; Lee, K.H.; Ahn, M.-J.; Luft, A.; van den Heuvel, M.; Cobo, M.; Smolin, A.; Vicente, D.; et al. LBA6Durvalumab with or without Tremelimumab vs Platinum-Based Chemotherapy as First-Line Treatment for Metastatic Non-Small Cell Lung Cancer: MYSTIC. Ann. Oncol. 2018, 29 (Suppl. 10). [CrossRef]

141. Morris, V.K.; Salem, M.E.; Nimeiri, H.; Iqbal, S.; Singh, P.; Ciombor, K.; Polite, B.; Deming, D.; Chan, E.; Wade, J.L.; et al. Nivolumab for Previously Treated Unresectable Metastatic Anal Cancer (NCI9673): A Multicentre, Single-Arm, Phase 2 Study. Lancet Oncol. 2017, 18, 446-453. [CrossRef]

142. Kang, Y.-K.; Boku, N.; Satoh, T.; Ryu, M.-H.; Chao, Y.; Kato, K.; Chung, H.C.; Chen, J.-S.; Muro, K.; Kang, W.K.; et al. Nivolumab in Patients with Advanced Gastric or Gastro-Oesophageal Junction Cancer Refractory to, or Intolerant of, at Least Two Previous Chemotherapy Regimens (ONO-4538-12, ATTRACTION-2): A Randomised, Double-Blind, Placebo-Controlled, Phase 3 Trial. Lancet 2017, 390, 2461-2471. [CrossRef]

143. Reardon, D.A.; Omuro, A.; Brandes, A.A.; Rieger, J.; Wick, A.; Sepulveda, J.; Phuphanich, S.; De Souza, P.; Ahluwalia, M.S.; Lim, M.; et al. OS10.3 Randomized Phase 3 Study Evaluating the Efficacy and Safety of Nivolumab vs Bevacizumab in Patients with Recurrent Glioblastoma: CheckMate 143. Neuro-oncology 2017, 19 (Suppl. 3), iii21. [CrossRef]

144. Primary Endpoint Not Met in Phase III CheckMate498 Trial of MGMT-Unmethylated GBM. Available online: https://www.targetedonc.com/news/primary-endpoint-not-met-in-phase-iii-checkmate498-trial-ofmgmtunmethylated-gbm (accessed on 11 October 2019).

145. Yau, T.; Park, J.W.; Finn, R.S.; Cheng, A.-L.; Mathurin, P.; Edeline, J.; Kudo, M.; Han, K.-H.; Harding, J.J.; Merle, P.; et al. CheckMate 459: A randomized, multi-center phase III study of nivolumab (NIVO) vs sorafenib (SOR) as first-line (1L) treatment in patients (pts) with advanced hepatocellular carcinoma (aHCC). Ann. Oncol. 2019, 30, mdz394.029. [CrossRef]

146. Weber, J.; Mandala, M.; Del Vecchio, M.; Gogas, H.J.; Arance, A.M.; Cowey, C.L.; Dalle, S.; Schenker, M.; Chiarion-Sileni, V.; Marquez-Rodas, I.; et al. Adjuvant Nivolumab versus Ipilimumab in Resected Stage III or IV Melanoma. N. Engl. J. Med. 2017, 377, 1824-1835. [CrossRef] [PubMed]

147. Scherpereel, A.; Mazieres, J.; Greillier, L.; Lantuejoul, S.; Dô, P.; Bylicki, O.; Monnet, I.; Corre, R.; Audigier-Valette, C.; Locatelli-Sanchez, M.; et al. Nivolumab or Nivolumab plus Ipilimumab in Patients with Relapsed Malignant Pleural Mesothelioma (IFCT-1501 MAPS2): A Multicentre, Open-Label, Randomised, Non-Comparative, Phase 2 Trial. Lancet Oncol. 2019, 20, 239-253. [CrossRef]

148. Rizvi, N.A.; Mazières, J.; Planchard, D.; Stinchcombe, T.E.; Dy, G.K.; Antonia, S.J.; Horn, L.; Lena, H.; Minenza, E.; Mennecier, B.; et al. Activity and Safety of Nivolumab, an Anti-PD-1 Immune Checkpoint Inhibitor, for Patients with Advanced, Refractory Squamous Non-Small-Cell Lung Cancer (CheckMate 063): A Phase 2, Single-Arm Trial. Lancet Oncol. 2015, 16, 257-265. [CrossRef]

149. Carbone, D.P.; Reck, M.; Paz-Ares, L.; Creelan, B.; Horn, L.; Steins, M.; Felip, E.; van den Heuvel, M.M.; Ciuleanu, T.-E.; Badin, F.; et al. First-Line Nivolumab in Stage IV or Recurrent Non-Small-Cell Lung Cancer. N. Engl. J. Med. 2017, 376, 2415-2426. [CrossRef] 
150. Motzer, R.J.; Rini, B.I.; McDermott, D.F.; Redman, B.G.; Kuzel, T.M.; Harrison, M.R.; Vaishampayan, U.N.; Drabkin, H.A.; George, S.; Logan, T.F.; et al. Nivolumab for Metastatic Renal Cell Carcinoma: Results of a Randomized Phase II Trial. J. Clin. Oncol. 2015, 33, 1430-1437. [CrossRef]

151. Bellmunt, J.; De Wit, R.; Vaughn, D.J.; Fradet, Y.; Lee, J.-L.; Fong, L.; Vogelzang, N.J.; Climent, M.A.; Petrylak, D.P.; Choueiri, T.K.; et al. Pembrolizumab as Second-Line Therapy for Advanced Urothelial Carcinoma. N. Engl. J. Med. 2017, 376, 1015-1026. [CrossRef]

152. Balar, A.V.; Castellano, D.; O’Donnell, P.H.; Grivas, P.; Vuky, J.; Powles, T.; Plimack, E.R.; Hahn, N.M.; Wit, R.D.; Pang, L.; et al. First-Line Pembrolizumab in Cisplatin-Ineligible Patients with Locally Advanced and Unresectable or Metastatic Urothelial Cancer (KEYNOTE-052): A Multicentre, Single-Arm, Phase 2 Study. Lancet Oncol. 2017, 18, 1483-1492. [CrossRef]

153. O’Donnell, P.H.; Balar, A.V.; Vuky, J.; Castellano, D.E.; Bellmunt, J.; Powles, T.; Bajorin, D.F.; Grivas, P.; Hahn, N.M.; Plimack, E.R.; et al. KEYNOTE-052: Phase 2 Study Evaluating First-Line Pembrolizumab (Pembro) in Cisplatin-Ineligible Advanced Urothelial Cancer (UC)—Updated Response and Survival Results. J. Clin. Oncol. 2019, 37 (Suppl. 15), 4546. [CrossRef]

154. Shah, M.A.; Kojima, T.; Hochhauser, D.; Enzinger, P.; Raimbourg, J.; Hollebecque, A.; Lordick, F.; Kim, S.-B.; Tajika, M.; Kim, H.T.; et al. Efficacy and Safety of Pembrolizumab for Heavily Pretreated Patients with Advanced, Metastatic Adenocarcinoma or Squamous Cell Carcinoma of the Esophagus: The Phase 2 KEYNOTE-180 Study. JAMA Oncol. 2019, 5, 546-550. [CrossRef] [PubMed]

155. Kojima, T.; Muro, K.; Francois, E.; Hsu, C.-H.; Moriwaki, T.; Kim, S.-B.; Lee, S.-H.; Bennouna, J.; Kato, K.; Lin, S.; et al. Pembrolizumab versus Chemotherapy as Second-Line Therapy for Advanced Esophageal Cancer: Phase III KEYNOTE-181 Study. J. Clin. Oncol. 2019, 37 (Suppl. 4), 2. [CrossRef]

156. Rischin, D.; Harrington, K.J.; Greil, R.; Soulieres, D.; Tahara, M.; De Castro, G.; Psyrri, A.; Baste, N.; Neupane, P.C.; Bratland, A.; et al. Protocol-Specified Final Analysis of the Phase 3 KEYNOTE-048 Trial of Pembrolizumab (Pembro) as First-Line Therapy for Recurrent/Metastatic Head and Neck Squamous Cell Carcinoma (R/M HNSCC). J. Clin. Oncol. 2019, 37 (Suppl. 15), 6000. [CrossRef]

157. Le, D.T.; Kavan, P.; Kim, T.W.; Burge, M.E.; Van Cutsem, E.; Hara, H.; Boland, P.M.; Van Laethem, J.-L.; Geva, R.; Taniguchi, H.; et al. KEYNOTE-164: Pembrolizumab for Patients with Advanced Microsatellite Instability High (MSI-H) Colorectal Cancer. J. Clin. Oncol. 2018, 36 (Suppl. 15), 3514. [CrossRef]

158. Hsu, C.; Lee, S.-H.; Ejadi, S.; Even, C.; Cohen, R.B.; Le Tourneau, C.; Mehnert, J.M.; Algazi, A.; van Brummelen, E.M.J.; Saraf, S.; et al. Safety and Antitumor Activity of Pembrolizumab in Patients with Programmed Death-Ligand 1-Positive Nasopharyngeal Carcinoma: Results of the KEYNOTE-028 Study. J. Clin. Oncol. 2017, 35, 4050-4056. [CrossRef]

159. Reck, M.; Rodríguez-Abreu, D.; Robinson, A.G.; Hui, R.; Csőszi, T.; Fülöp, A.; Gottfried, M.; Peled, N.; Tafreshi, A.; Cuffe, S.; et al. Pembrolizumab versus Chemotherapy for PD-L1-Positive Non-Small-Cell Lung Cancer. N. Engl. J. Med. 2016, 375, 1823-1833. [CrossRef]

160. Herbst, R.S.; Baas, P.; Kim, D.-W.; Felip, E.; Pérez-Gracia, J.L.; Han, J.-Y.; Molina, J.; Kim, J.-H.; Arvis, C.D.; Ahn, M.-J.; et al. Pembrolizumab versus Docetaxel for Previously Treated, PD-L1-Positive, Advanced Non-Small-Cell Lung Cancer (KEYNOTE-010): A Randomised Controlled Trial. Lancet 2016, 387, 1540-1550. [CrossRef]

161. Gandhi, L.; Rodríguez-Abreu, D.; Gadgeel, S.; Esteban, E.; Felip, E.; De Angelis, F.; Domine, M.; Clingan, P.; Hochmair, M.J.; Powell, S.F.; et al. Pembrolizumab plus Chemotherapy in Metastatic Non-Small-Cell Lung Cancer. N. Engl. J. Med. 2018, 378, 2078-2092. [CrossRef]

162. Paz-Ares, L.; Luft, A.; Vicente, D.; Tafreshi, A.; Gümüş, M.; Mazières, J.; Hermes, B.; Şenler, F.Ç.; Csőszi, T.; Fülöp, A.; et al. Pembrolizumab plus Chemotherapy for Squamous Non-Small-Cell Lung Cancer. N. Engl. J. Med. 2018. [CrossRef]

163. Reck, M.; Rodríguez-Abreu, D.; Robinson, A.G.; Hui, R.; Csőszi, T.; Fülöp, A.; Gottfried, M.; Peled, N.; Tafreshi, A.; Cuffe, S.; et al. Updated Analysis of KEYNOTE-024: Pembrolizumab Versus Platinum-Based Chemotherapy for Advanced Non-Small-Cell Lung Cancer With PD-L1 Tumor Proportion Score of 50\% or Greater. J. Clin. Oncol. 2019, 37, 537-546. [CrossRef]

164. Ott, P.A.; Elez, E.; Hiret, S.; Kim, D.-W.; Morosky, A.; Saraf, S.; Piperdi, B.; Mehnert, J.M. Pembrolizumab in Patients with Extensive-Stage Small-Cell Lung Cancer: Results From the Phase Ib KEYNOTE-028 Study. J. Clin. Oncol. 2017, 35, 3823-3829. [CrossRef] 
165. Chung, H.C.; Piha-Paul, S.A.; Lopez-Martin, J.; Schellens, J.H.M.; Kao, S.; Miller, W.H.; Delord, J.-P.; Gao, B.; Planchard, D.; Gottfried, M.; et al. Abstract CT073: Pembrolizumab after Two or More Lines of Prior Therapy in Patients with Advanced Small-Cell Lung Cancer (SCLC): Results from the KEYNOTE-028 and KEYNOTE-158 Studies. Cancer Res. 2019, 79 (Suppl. 13), CT073. [CrossRef]

166. Balar, A.V.; Galsky, M.D.; Rosenberg, J.E.; Powles, T.; Petrylak, D.P.; Bellmunt, J.; Loriot, Y.; Necchi, A.; Hoffman-Censits, J.; Perez-Gracia, J.L.; et al. Atezolizumab as First-Line Treatment in Cisplatin-Ineligible Patients with Locally Advanced and Metastatic Urothelial Carcinoma: A Single-Arm, Multicentre, Phase 2 Trial. Lancet 2017, 389, 67-76. [CrossRef]

167. Sullivan, R.J.; Hamid, O.; Gonzalez, R.; Infante, J.R.; Patel, M.R.; Hodi, F.S.; Lewis, K.D.; Tawbi, H.A.; Hernandez, G.; Wongchenko, M.J.; et al. Atezolizumab plus Cobimetinib and Vemurafenib in BRAF -Mutated Melanoma Patients. Nat. Med. 2019, 25, 929-935. [CrossRef] [PubMed]

168. Rittmeyer, A.; Barlesi, F.; Waterkamp, D.; Park, K.; Ciardiello, F.; von Pawel, J.; Gadgeel, S.M.; Hida, T.; Kowalski, D.M.; Dols, M.C.; et al. Atezolizumab versus Docetaxel in Patients with Previously Treated Non-Small-Cell Lung Cancer (OAK): A Phase 3, Open-Label, Multicentre Randomised Controlled Trial. Lancet 2017, 389, 255-265. [CrossRef]

169. Barlesi, F.; Vansteenkiste, J.; Spigel, D.; Ishii, H.; Garassino, M.; De Marinis, F.; Özgüroğlu, M.; Szczesna, A.; Polychronis, A.; Uslu, R.; et al. Avelumab versus Docetaxel in Patients with Platinum-Treated Advanced Non-Small-Cell Lung Cancer (JAVELIN Lung 200): An Open-Label, Randomised, Phase 3 Study. Lancet Oncol. 2018, 19, 1468-1479. [CrossRef]

170. Powles, T.; O’Donnell, P.H.; Massard, C.; Arkenau, H.-T.; Friedlander, T.W.; Hoimes, C.J.; Lee, J.L.; Ong, M.; Sridhar, S.S.; Vogelzang, N.J.; et al. Efficacy and Safety of Durvalumab in Locally Advanced or Metastatic Urothelial Carcinoma: Updated Results from a Phase 1/2 Open-Label Study. JAMA Oncol. 2017, 3, e172411. [CrossRef]

171. Nowak, A.K.; Lesterhuis, W.J.; Hughes, B.G.M.; Brown, C.; Kok, P.S.; O’Byrne, K.J.; John, T.; Pavlakis, N.; Kao, S.C.-H.; Yip, S.; et al. DREAM: A Phase II Study of Durvalumab with First Line Chemotherapy in Mesothelioma-First Results. J. Clin. Oncol. 2018, 36 (Suppl. 15), 8503. [CrossRef]

(C) 2019 by the authors. Licensee MDPI, Basel, Switzerland. This article is an open access article distributed under the terms and conditions of the Creative Commons Attribution (CC BY) license (http://creativecommons.org/licenses/by/4.0/). 